\title{
On Cohomology Operations
}

\author{
Tokushi Nakamura \\ (Recëived Oct. 14, 1963)
}

Introduction. The cohomology operation has been defined in two ways. The original "combinatorial" definition was given by Steenrod [18] and Thom [22]. This definition can be given in terms of the homology group of the symmetric group $\operatorname{Tor}^{Z(S(n))}(Z, Z)$ and gives us a way to compute cohomology operations (though difficult to carry out effectively except in simple cases). The other "axiomatic" definition was given by Eilenberg [9] and Serre [16] using the cohomology group of the Eilenberg-MacLane space $H^{*}(\Pi, q ; G)$. The cohomology operation under this definition has been object of important researches by Cartan [4], and Serre [16]. It is clear from the definition that these include the combinatorially defined cohomology operations, but it is easily seen from Cartan's results that these latter exhaust the former (Nakamura [11]).

Now a theorem of Dold-Thom [8] implies that the Eilenberg-MacLane space $K(\Pi, q)$ is obtained as the infinite symmetric product of a Moor space $M(\Pi, q)$. In the special case $\Pi=Z$, we have

$$
K(Z, q)=\lim _{n \rightarrow \infty} \underset{n}{\times} S^{q} / S(n) .
$$

Steenrod [19] has proved a more precise from of (1), i.e.

$$
H^{p}(Z, q)=\sum_{n=0}^{\infty} H^{p}\left(\underset{n}{\times} S^{q} / S(n), \underset{n-1}{\times} S^{q} / S(n-1)\right) .
$$

On the other hand, it is geometrically clear that we have

$$
\operatorname{Tor}_{i}^{Z(S(n))}(Z, Z)=\lim _{q \rightarrow \infty} H^{n q-i}\left(\underset{n}{\times} S^{q} / S(n)\right) \quad \text { for } q \equiv 0 \bmod 2 .
$$

We shall prove in Chapter 1 , a more precise form of (2), i.e.

$$
\operatorname{Tor}_{i}^{Z(S(n))}(Z, Z) \supset H^{n q-i}\left(\underset{n}{\times} S^{q} / S(n), \underset{n-1}{\times} S^{q} / S(n-1)\right) \quad \text { for } q \equiv 0 \bmod 2 .
$$

From $(1)^{\prime},(2)^{\prime}$ it follows in particular that the combinatorially defined cohomology operations exhaust the axiomatically defined ones. Dold [6] has also proved $(2)^{\prime}$ and thus shown the equivalence of the two definitions of cohomology operations. In Nakamura [12], we have given another way to prove $(2)^{\prime}$, which is more convenient in view of generalizations to the case of cohomology operations of higher orders, without giving however the details of proof. In Chapter 1, of this paper, we shall give the proof in all detail, which is simplified by using the argument due to Dold [6].

In Chapter 2, we shall give a structure of $C W$-complex $C\left(\underset{n}{\times} S^{q}\right)$ to $\underset{n}{\times} S^{q}$ such that 


$$
\begin{gathered}
\bar{B}^{(q-1)}\left(K(N, 1)_{N}\right)^{p}=\lim _{n \rightarrow \infty} C^{p}\left(\underset{n}{\times} S^{q} / S(n)\right) \\
W(S(n))_{i}=\lim _{q \rightarrow \infty} C^{n q-i}\left(\underset{n}{\times} S^{q}, \bigcup_{\nu=0}^{n}\left(\underset{\nu-1}{\times} S^{q}\right) \times \infty \times\left(\underset{n-\nu}{\times} S^{q}\right)\right) \quad \text { for } q \equiv 0 \bmod 2
\end{gathered}
$$

where $\bar{B}^{(q-1)}$ means $(q-1)$-fold bar construction of the normalized $F D$-module $K(N, 1)_{N}, N$ being the semigroup of nonnegative integers, and $W(S(n))$ means a free projective resolution of $Z$ over the group ring of the symmetric group $S(n)$.

More precisely, we shall prove.

$$
\begin{gathered}
\bar{B}^{(q)}(Z(N))^{p}=\sum_{n=0}^{\infty} C^{p}\left(\underset{n}{\times} S^{q} / S(n), \underset{n-1}{\times} S^{q} / S(n-1)\right) \\
W(S(n))_{i} \supset W(n, q)_{i} \simeq C^{n q-i}\left(\underset{n}{\times} S^{q}, \underset{\nu=0}{\cup}\left(\underset{\nu-1}{\times} S^{q}\right) \times \infty \times\left(\underset{n-\nu}{\times} S^{q}\right)\right)
\end{gathered}
$$

from which $\left(1^{\prime}\right)$ and $\left(2^{\prime}\right)$ will easily follow. Thus this Chapter will give another proof for the equivalence of two definitions, which is more algebraic and independent of Dold-Thom theorem.

In Chapter 3, we shall consider cohomology operations of the second order. From the combinatorial side, we have only Adem's operation hitherto introduced among these. We shall indicate the way to define combinatorially the cohomology operation of the second order, which will exhaust, like in case of cohomology operations of the first order, all the axiomatically defined operations.

\section{Chapter 1. Equivalence of two definitions of cohomology operations}

In the first two sections of Chapter 1 , I have revised the definitions of such known notions as $F D$-algebras and d.g.a. algebras. These notions were introduced by Eilenberg [9] and Cartan [4] and also studied by Dold [5]. But the meaning of the $F D$-algebra differs a little when used in [9] or [5]. So we have given our precise definition to begin with. We have gathered the known results about these notations to be used later on in these two sections. Apart from these our paper is self-contained.

\section{$\S 1$. The W-construction}

In the following, the term module means a module over a fixed commutative ring $\Lambda$ with unit 1 .

An $F D$-module $K$ is a module which is a direct sum of the $p$-chain modules $K_{p}$ defined for each non-negative integer $p$ on which the $i$-th face operator $\partial_{i}: K_{p} \rightarrow K_{p-1}$ and the $i$-th degeneracy operator $s_{i}: K_{p} \rightarrow K_{p+1}$ are defined so as to satisfy the well-known formulae (See Dold [5]).

Let $K$ and $K^{\prime}$ be two $F D$-modules. Then an $F D$-homomorphism $f: K \rightarrow K^{\prime}$ is a homomorphism such that $f\left(K_{p}\right) \subset K_{p}^{\prime}$ and $\partial_{i} f=f \partial_{i}, s_{i} f=f s_{i}$.

Let $\Delta(r)$ be an $F D$-module whose $p$-chain module is a free module generated by non-decreasing sequences of integers $\left(a_{0}, a_{1}, \cdots, a_{p}\right)$ such that $0 \leqq a_{0} \leqq a_{1} \leqq \cdots \leqq$ $a_{p} \leqq r$ with $\partial_{i}$ and $s_{i}$ defined by 


$$
\begin{aligned}
& \partial_{i}\left(a_{0}, \cdots, a_{p}\right)=\left(a_{0}, \cdots, \hat{a}_{i}, \cdots, a_{p}\right) \\
& s_{i}\left(a_{0}, \cdots, a_{p}\right)=\left(a_{0}, \cdots, a_{i}, a_{i}, \cdots a_{p}\right) .
\end{aligned}
$$

An $F D$-module is called augmented, if we have a surjective homomorphism $\varepsilon: K \rightarrow \Delta(0)$, called augmentation.

Let $K$ and $K^{\prime}$ be two augmented $F D$-modules. Then an $F D$-homomorphism $f: K \rightarrow K^{\prime}$ means an $F D$-homomorphism preserving the augmentation.

Let $K$ and $K^{\prime}$ be two $F D$-modules. Then the cartesian product $K \times K^{\prime}$ of $K$ and $K^{\prime}$ is defined by $\left(K \times K^{\prime}\right)_{p}=K_{p} \otimes K_{p}^{\prime}$ and $\partial_{i}=\partial_{i} \otimes \partial_{i}, s_{i}=s_{i} \otimes s_{i}$.

An $F D$-algebra $K$ is an $F D$-module on which there is defined an $F D$-homomorphism $m: K \times K \rightarrow K$ called the multiplication where $m$ is assumed to be associative and commutative.

Let $K$ and $K^{\prime}$ be two $F D$-algebras. Then a $F D$-homomorphism $f: K \rightarrow K^{\prime}$ is an $F D$-homomorphism preserving the multiplication.

Obviously $\Delta(0)$ has the structure of an $F D$-algebra.

An $F D$-algebra $K$ is called augmented, if there exists a surjective $F D$ homomorphism $\varepsilon: K \rightarrow \Delta(0)$ between $F D$-algebras.

An $F D$-homomorphism $f: K \rightarrow K^{\prime}$ between two augmented $F D$-algebras is like-wise defined.

An augmented $F D$-algebra $K$ is called unitary, if there exists a unit $1_{p}$ in each $K_{p}$ such that $\varepsilon\left(1_{p}\right)=(0, \cdots, 0)$ augmented $F D$-algebra is called connected, if $\varepsilon: K_{0} \cong \Lambda(0)$.

An $F D$-module $K$ is called free, if each $K_{p}$ is a free module with free generators called $p$-simplices and $\partial_{i}$ and $s_{i}$ transform each simplex into a simplex or 0 . When we speak of an $F D$-homomorphism $f: K \rightarrow K^{\prime}$, where $K$ and $K^{\prime}$ are free $F D$-modules, we always understand that $f$ transforms a simplex into a simplex or 0 . The cartesian product $K \times K^{\prime}$ of free $F D$-modules $K$ and $K^{\prime}$ means a free $F D$-module whose simplices are the product of the simplices of $K$ and the simlices of $K^{\prime}$. An $F D$-algebra $K$ is called free, if $K$ is a free $F D$-module and the multiplication $m: K \times K \rightarrow K$ means an $F D$-homomorphism between free $F D$-modules. In particular, a unitary free $F D$-algebra means a unitary $F D$ algebra having specified simplices as units $1_{p}$. Later on, the term free will be used under appropriate modifications also in other cases. We shall not repea the detailed explanation of its meaning in each case, as it will be clear by analogy with this case.

An augmented $F D$-module $W$ over an augmented $F D$-algebra $K$ is an augmented $F D$-module on which an $F D$-homomorphism $m: K \times W \rightarrow W$ is defined so as to be associative with respect to the multiplication of $K$, that is, $\left(\sigma \cdot \sigma^{\prime}\right) \cdot \tau=$ $\sigma \cdot\left(\sigma^{\prime} \cdot \tau\right)$ for $\sigma, \sigma^{\prime} \in K_{p}$ and $\tau \in W_{p}$.

Suppose given an augmented $F D$-algebra $K$. Then we denote by $I(K)$ the ideal of $K$ consisting of elements $\sigma \in K$ with $\varepsilon(\sigma)=0$. Let $W$ be an augmented $F D$-module over an augmented $F D$-algebJa $K$. Then we define an augmented $F D$-module $\bar{W}$ over $K$ to be the factor module $W / I(K) W$. The notation $\tilde{\omega}: W \rightarrow \bar{W}$ means a natural projection. If $K$ is unitary, the action of $K$ on $\bar{W}$ is trivial.

Let $W$ and $W^{\prime}$ be two augmented $F D$-modules over two augmented $F D$ algebras $K$ and $K^{\prime}$ respectively. Supsose given an $F D$-homomorphism $f: K \rightarrow K^{\prime}$. Then an $F D$-homorphism $\tilde{f}: W \rightarrow W^{\prime}$ compatible with $f$ is one which satisfies 
the relation $\hat{f}(\sigma \cdot \tau)=f(\sigma) \tilde{f}(\tau)$ for $\sigma \in K_{p}$ and $\tau \in W_{p}$. Clearly $\tilde{f}$ determines an $F D$. homomorphism $\bar{f}: \bar{W} \rightarrow \bar{W}^{\prime}$ by the property $\bar{f} \tilde{\omega}=\tilde{\omega} \tilde{f}$.

From now on, we consider a fixed unitary $F D$-algebra $K$. Then the $W$ construction of $K$ is an augmented $F D$-module over $K$ whose $p$-chain module is defined by $(W K)_{p}=K_{p} \otimes K_{p-1} \otimes \cdots \otimes K_{0}$ equipped with the trivial multiplication from the left. As usual, we use the notation $\sigma\left\langle\sigma_{p-1}, \cdots, \sigma_{0}\right\rangle$ or simply $\sigma \times \tau$ to indicate the elements of $W K$ corresponding to the tensor products $\sigma \otimes \sigma_{p-1} \otimes \cdots \otimes \sigma_{0}$ and $\partial_{i}$ and $s_{i}$ is introduced so as to satisfy the relatian $\partial_{0} s=i d, \partial_{1} s=1_{0}$ in dimension $p=0$ and $\partial_{i+1} s=s \partial_{i}$ for $i \geqq 0$ in dimension $p>0$ and $s_{0} s=s s, s_{i+1} s=s s_{i}$ where the suspension operator $s$ is defined by $s\left(\sigma\left\langle\sigma_{p-1}, \cdots, \sigma_{0}\right\rangle\right)=\left\langle\sigma, \sigma_{p-1}, \cdots, \sigma_{0}\right\rangle$. The augmentation $\varepsilon$ is defined by $\varepsilon(\sigma \times \tau)=\varepsilon(\sigma) \cdot \varepsilon(\tau)$. We remind here the following formulae on $\partial_{i}$ and $\mathrm{s}_{i}$

$$
\begin{array}{rlrl}
\partial_{0}\left(\sigma\left\langle\sigma_{p-1}, \cdots, \sigma_{0}\right\rangle\right) & =\partial_{0} \sigma \cdot \sigma_{p-1}\left\langle\sigma_{p-2}, \cdots, \sigma_{0}\right\rangle & \\
\partial_{i}\left(\sigma\left\langle\sigma_{p-1}, \cdots, \sigma_{0}\right\rangle\right) & =\partial_{i} \sigma\left\langle\partial_{i-1} \sigma_{p-1}, \cdots, \partial_{0} \sigma_{p-i} \cdot \sigma_{p-i-1}, \cdots, \sigma_{0}\right\rangle & & \text { for } 0<i<p \\
\partial_{p}\left(\sigma\left\langle\sigma_{p-1}, \cdots, \sigma_{0}\right\rangle\right) & =\partial_{p} \sigma \cdot \varepsilon\left(\sigma_{p}\right)\left\langle\partial_{p-1} \sigma_{p-1}, \cdots, \partial_{1} \sigma_{1}\right\rangle & \\
s_{i}\left(\sigma\left\langle\sigma_{p-1}, \cdots, \sigma_{0}\right\rangle\right) & =s_{i} \sigma\left\langle s_{i-1} \sigma_{p-1}, \cdots, s_{0} \sigma_{p-i}, 1_{p-i}, \cdots, \sigma_{0}\right\rangle & & \text { for } 0 \leqq i \leqq p .
\end{array}
$$

Let $K$ be an augmented $F D$-algebra. We say that a multiplicity $\mu$ is defined on $K$, if we have a decomposition of $K$ into a direct sum of the $F D$-submodules, each of which is not necessarily augmented, ${ }_{l} K$ indexed by non-negative integers $l$ such that ${ }_{l} K=\sum_{p=0}^{\infty}{ }_{l} K_{p}$ with ${ }_{l} K_{p}={ }_{l} K \cap K_{p},{ }_{l} K \cdot{ }_{m} K \subset{ }_{l+m} K, \partial_{i}\left({ }_{l} K\right) \subset{ }_{l} K, s_{i}\left({ }_{l} K\right) \subset{ }_{l} K$ and $\varepsilon \mid{ }_{l} K=0$ for $l>0$. An element $\sigma$ of ${ }_{l} K$ is called a homogeneous element of multiplicity $\mu(\sigma)=l$. Then we define a multiplicity $\mu(\sigma)$ of $\sigma$ to be the maximum of $l$ such that $\sigma$ has a nonzero component in the direct summand ${ }_{l} K$ of $K$. Now all the elements of $K$ with multiplicity $\leqq n$ generate an augmented $F D$-submodule of $K$ which will be denoted by $K(n)$. An augmented $F D$-algebra $K$ with multiplicity is called free, if each component ${ }_{l} K_{p}$ is free.

If a multiplicity $\mu$ is defined on $K$. Then $\mu$ is extended to a multiplicity $\tilde{\mu}$ on $W K$ by setting $\tilde{\mu}\left(\sigma\left\langle\sigma_{p-1}, \cdots, \sigma_{0}\right\rangle\right)=\mu(\sigma)+\sum_{i=0}^{p-1} \mu\left(\sigma_{i}\right)$. Here we note that we can define two functions on $W K, \mu_{F}$ called the fibre multiplicity, and $\mu_{B}$ called the base multiplicity, defined by $\mu_{F}\left(\sigma\left\langle\sigma_{p-1}, \cdots, \sigma_{0}\right\rangle\right)=\mu(\sigma)$ and $\mu_{B}\left(\sigma\left\langle\sigma_{p-1}, \cdots, \sigma_{0}\right\rangle\right)=$ $\mu\left\langle\sigma_{p-1}, \cdots, \sigma_{0}\right\rangle$ respectively.

The $F D$-homomorphism $f: K \rightarrow K^{\prime}$ between two $F D$-algebras $K$ and $K^{\prime}$ with multiplicities $\mu$ is supposed to obey the rule $\mu f=\mu$. Now $f$ can be extended to an $F D$-homomorphism $\tilde{f}: W K \rightarrow W K^{\prime}$ compatible with $f$ and we have $\bar{f}: \bar{W} K \rightarrow \bar{W} K^{\prime}$. Clearly a multiplicity is defined on $K \times K^{\prime}$ in a natural way from the multiplication $m: K \times K \rightarrow K$ is multiplicity preserving, and so it can be extended to the multiplication $\tilde{m}: W K \times W K \rightarrow W K$ which gives rise to the structure of a unitary $F D$-algebra with multiplicity on $W K$. As easily seen $K$ is a subalgebra of $W K$ and $\bar{W} K=W K / I(K) W K$ is a unitary connected $F D$-algebra with multiplicity.

Let $F_{m}$ be the submodule of $W K$ generated by the elements whose base multiplicity $\mu_{B} \leqq m$. Then the family of $F_{m}$ defines a filtration on $W K$. We denote $F_{n-m, m}={ }_{n} W K \cap F_{m}$. $E_{l, m}^{0}(F)$ will mean $F_{l, m} / F_{l+1, m-1}$.

Proposision. 1.1. Let $K$ be a unitary $F D$-algebra such that $\varepsilon:{ }_{0} K_{p} \cong \Lambda 1_{p}$. Then we have 


$$
E_{l, m}^{0}(F) \cong_{l} K \times_{m} \bar{W} K
$$

Rroof. Obviously $\mu\left(\partial_{0} \sigma \cdot \sigma_{p-1}\right)=\mu(\sigma)$ holds, if and only if $\mu\left(\sigma_{p-1}\right)=0$. From the assumption, this is equivalent to say $\sigma_{p-1}=\lambda 1_{p-1}$ for some $\lambda$. Thus we have

$$
\begin{aligned}
\partial_{0}\left(\sigma\left\langle\sigma_{p-1}, \cdots, \sigma_{0}\right\rangle\right) & =\partial_{0} \sigma \cdot \sigma_{p-1}\left\langle\sigma_{p-2}, \cdots, \sigma_{0}\right\rangle \\
& =\partial_{0} \sigma \cdot \lambda\left\langle\sigma_{p-2}, \cdots, \sigma\right\rangle \\
& =\partial_{0} \sigma \cdot \tilde{\omega} \partial_{0}\left\langle\sigma_{p-1}, \cdots, \sigma_{0}\right\rangle .
\end{aligned}
$$

On the other hand, the definition of $\partial_{0}$ implies

$$
\begin{aligned}
\partial_{i}\left(\sigma\left\langle\sigma_{p-1}, \cdots, \sigma_{0}\right\rangle\right) & =\partial_{i} \sigma \cdot \partial_{i}\left\langle\sigma_{p-1}, \cdots, \sigma_{0}\right\rangle \\
& =\partial_{i} \sigma \cdot \tilde{\omega} \partial_{i}\left\langle\sigma_{p-1}, \cdots, \sigma_{0}\right\rangle
\end{aligned}
$$

for $i>0$. Let $\sigma\left\langle\sigma_{p-1}, \cdots, \sigma_{0}\right\rangle$ be an element of $F_{l, m}$. Then we have

$$
\partial_{i}\left(\sigma\left\langle\sigma_{p-1}, \cdots, \sigma_{0}\right\rangle\right) \equiv \partial_{i} \sigma \cdot \tilde{\omega} \partial_{i}\left\langle\sigma_{p-1}, \cdots, \sigma_{0}\right\rangle
$$

$\bmod F_{l+1, m-1}$ for all $i$. This concludes the proof.

Let $I I$ be an abelian semi-group. Then a unitary free $F D$-algebra $K(\Pi, q)$ is defined inductively as follows: If $q=0, K(\Pi, q)_{p}=\Lambda(\Pi)$, for $p \geqq 0$ and $\partial_{i}=s_{i}=i d$ for $p>0$ and if $q \geqq 1, K(\Pi, q)=\bar{W} K(\Pi, q-1)$.

Let us denote by $N$ the semi-group of non-negative integers with respect to its addition. Then the multiplicity $\mu$ is defined on $K(N, 0)_{p}=\Lambda(N)$ by the rule $\mu^{\prime}\left(\alpha^{l}\right)=l$ where $\alpha$ denotes the generator of $N$. Since $\Lambda(N)$ is isomorphic to the polynomial algebra $\Lambda[\alpha]$ over $\Lambda$ with one generator $\alpha,{ }_{l} K(N, 0)_{p}$ is defined to be $\Lambda\left((\alpha-e)^{l}\right)$ with $e=\alpha^{0}$. To simplify the notation, we write in the sequel $K(N, q)(n)$ as $K(N, q, n)$, and $W(N, q)(n)$ as $W(N, q, n)$ and so on.

Replacing $K$ by $K(N, q)$ in the previous Proposition, we have

Proposition 1.2.

$$
\begin{aligned}
E_{l, m}^{0}(F) & \cong l K(N, q) \times{ }_{m} K(N, q+1) \\
& \cong K(N, q, l) / K(N, q, l-1) \times K(N, q+1, m) / K(N, q+1, m-1) .
\end{aligned}
$$

Let $S(n)$ be the symmetric group of degree $n$ and $\Pi$ be a subgroup of $S(n)$. Then $I I$ operates on the $n$-th tensor product $\otimes M$ of a module $M$ and the tensor product $\otimes \operatorname{Hom}\left(M, M^{\prime}\right)$ of a module $\operatorname{Hom}\left(\stackrel{n}{M}, M^{\prime}\right)$ of homomorphisms $f: M \rightarrow M^{\prime}$ etc. as the permutation of factors, so the quotient $\otimes_{n} M / I I$ is defined. (See Dold [5]). Let $K$ be an $F D$-module. We define $\times K / \Pi$ be $(\times K / \Pi)_{p}=\bigotimes_{n} K_{p} / \Pi, \partial_{i}=$ $\bigotimes_{n} \partial_{i} / \Pi, s_{i}=\bigotimes_{n} s_{i} / \Pi$. In the special case $\Pi \stackrel{n}{=} S(n), \underset{n}{\times} K / S(n)$ will be denoted by

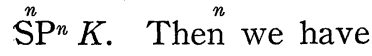

Proposition 1.3.

$$
{ }_{n} K(N, q) \cong \mathrm{SP}^{n}{ }_{1} K(N, q) \text {. }
$$

Proof. For the proof, we only need to consider the multiplication. (See Nakamura [12])

$$
m: \underset{n}{\times}{ }_{1} K(N, q) \rightarrow{ }_{n} K(N, q) .
$$

Corollary 1.4.

$$
K(N, q, n) \cong \mathrm{SP}^{n} K(N, q, 1)
$$


Proof. Since we have the direct sum decomposition $K(N, q 1)={ }_{1} K(N, q)+$ ${ }_{0} K(N, q)$, the Steenrod formula

$$
\mathrm{SP}^{n}\left(K+K^{\prime}\right)=\sum_{l+m=n} \mathrm{SP}^{l} K \times \mathrm{SP}^{m} K^{\prime}
$$

and the formula

$$
{ }_{l} K(N, q) \cong \mathrm{SP}^{l}{ }_{1} K(N, q)
$$

provide the required result.

In the some way, we have

Proposition 1.5.

$$
\begin{aligned}
{ }_{n} W(N, q) & \cong \mathrm{SP}^{n}{ }_{1} W(N, q) \\
W(N, q, n) & \cong \mathrm{SP}^{n} W(N, q, 1)
\end{aligned}
$$

In the rest of this section, we restrict ourselves to the case $\Lambda=Z$. Let $K(X)$ be a singular complex of a topological space $X$. Easy computation shows

Proposition 1.6.

$$
K(N, q, 1) \simeq K\left(S^{q}\right) .
$$

Making use of the geometric realization of semi-simplicial complexes, we have

Proposition 1.7.

$$
K(N, q, n) \simeq K\left(\mathrm{SP}^{n} S^{q}\right) .
$$

Let $M(Z, q)$ be a free $F D$-module whose normalized $F D$-module has a singlegenerator in dimension $q$. Then we have

Proposition 1.8.

$$
{ }_{1} K(N, q) \cong M(Z, q) \text {. }
$$

In the same way, we have

Proposition 1.9.

$$
\begin{aligned}
W(N, q, 1) & \simeq K\left(E^{q+1}\right) \\
W(N, q, 1) & \simeq K\left(\mathrm{SP}^{n} E^{q+1}\right) \\
& W(N, q) \cong M(0, q) .
\end{aligned}
$$

The notation $M\left(Z_{\theta}, q\right)$ means free $F D$-module whose normalized $F D$-module has only two generators $\sigma$ and $\tau$ in dimension $q$ and $q+1$ respectively and has the homology group isomorphic to $Z_{\theta}$ in dimension $q$.

\section{§2. The bar-construction}

A graded module $M$ is a module which is a direct sum of the submodules. $M_{p}$ indexed by a nonnegative integer $p$ called degree or dimension. A graded algebra $A$ is a graded module $\sum A_{p}$ with the structure of algebra such that $A_{p} \cdot A_{r} \subset A_{p+r}$ which is assumed to be associative and anti-commutative.

A d.g.a. module $M$ is a graded module with a boundary operator $\partial$ such that $\partial\left(M_{p}\right) \subset M_{p-1}$ and $\partial \partial=0$, besides the augmentation $\varepsilon$ mapping $M$ onto $A$ satisfying the relation $\varepsilon \mid M_{p}=0$ for $p>0$ and $\varepsilon \partial=0$. A d.g.a. algebra $A$ is a 
d.g.a. module with the structure of graded algebra such that $\partial\left(a \cdot a^{\prime}\right)=\partial a \cdot a^{\prime}+$ $(-1)^{|a|} a \cdot \partial a^{\prime}$ for an element $a$ of dimension $|a|$ and $\varepsilon\left(a \cdot a^{\prime}\right)=\varepsilon a \cdot \varepsilon a^{\prime}$. A d.g.a. algebra is called unitary if there exists a unit 1 in $A_{0}$ such that $\varepsilon(1)=1$. A d.g.a. algebra is called connected, if $\varepsilon A_{0} \cong \Lambda$ :

A graded module $M$ is called free, if each $M_{p}$ is free. A d.g.a. module $M$ is called free if $\varepsilon^{-1}(0)$ is a free graded submodule of $M$.

A d.g.a. module $M$ over a d.g.a. algebra $A$ is a d.g.a. module on which a multiplication of $A$ from the left is defined so as to satsfy the relations $A_{p} \cdot M_{r} \subset M_{p+r}, \quad\left(a \cdot a^{\prime}\right) m=a \cdot\left(a^{\prime} m\right), \quad \partial(a \cdot m)=\partial a \cdot m+(-1)^{|a|} a \cdot \partial m$ and $\varepsilon(a \cdot m)=\varepsilon a \cdot \varepsilon m$ for $a, a^{\prime} \in A$ and $m \in M$.

Suppose given a d.g.a. algebra $A$. Then we denote by $I(A)$ or $\bar{A}$ the ideal of $A$ consisting of elements $a \in A$ with augmentation $\varepsilon(a)=0$. Let $M$ be a d.g.a. module over a d.g.a. algebra $A$. Then we define a d.g.a. module $\bar{M}$ over $A$ to be the factor module $M / I(A) M$. The notation $\tilde{\omega}: M \rightarrow \bar{M}$ means a natural projection. If $A$ is unitary, the action of $A$ on $\bar{M}$ is trivial.

Let $M$ and $M^{\prime}$ be two d.g.a. modules. Then a d.g.a. homomorphism $f: M \rightarrow M^{\prime}$ is a homomorphism such that $f\left(M_{p}\right) \subset M_{p}^{\prime} . \quad \partial f=f \partial$ and $\varepsilon f=\varepsilon$. Let $A$ and $A^{\prime}$ is two d.g.a. algebras. Then a d.g.a. homomorphism $f: A \rightarrow A^{\prime}$ preserves the structure of algebras.

Let $M$ and $M^{\prime}$ be two d.g.a. modules over d.g.a. algebras $A$ and $A^{\prime}$. Suppose given a d.g.a. homomorphism $f: A \rightarrow A^{\prime}$. Then a d.g.a. homomorphism $\tilde{f}: M \rightarrow M^{\prime}$ compatible with $f$ is one which satisfies the relation $\tilde{f}(a \cdot m)=f(a) \cdot \tilde{f}(m)$. Clearls $\tilde{f}$ determines a d.g.a. homomorphism $\tilde{f}: \bar{M} \rightarrow \bar{M}^{\prime}$ by the property $\tilde{f} \tilde{\omega}=\tilde{\omega} \tilde{f}$.

From now on, we consider a fixed unitary connected d.g.a. algebra $A$. We write $\otimes \bar{A}$ as $\bar{A}^{(\text {() }}$. Then the bar constrtction of $A$ is a d.g.a. module $B A=$ $A \otimes \sum_{l=0}^{\infty} \bar{A}^{(l)}$ over $A$ with respect to the multiplication from the left. In the usual manner, we use the notation $a\left[a_{1}|\cdots| a_{\imath}\right]$ or simply $a \otimes b$ etc. to indicate the element of $B A$ corresponding to the tensor product $a \otimes a_{1} \otimes \cdots a_{\iota}$ on the right hand side of the above equality. Then we define $\operatorname{dim}\left(a\left[a_{1}|\cdots| a\right]\right)=|a|+\sum_{i=1}^{i}\left|a_{i}\right|+\iota$ with $|a|=\operatorname{dim} a . \quad \partial$ is introduced so as to satisfy the relation $\partial s+s \partial=1-\varepsilon$ with respect to the suspension operator $s$ defined by $s\left(a\left[a_{1}|\cdots| a_{\imath}\right]\right)=\left[a\left|a_{1}\right| \cdots \mid a_{\imath}\right]$ and the augmentation $\varepsilon$ defined by $\varepsilon\left(a\left[a_{1}|\cdots| a_{l}\right]\right)=\varepsilon(a)$ if $\iota=0$.

Here we recall the boundary formula

$$
\begin{aligned}
\partial\left(a_{0}\left[a_{1}|\cdots| a_{\imath}\right]\right) \\
=\partial a_{0}\left[a_{1}|\cdots| a_{\imath}\right]+a_{0} a_{1}\left[a_{2}|\cdots| a_{\imath}\right] \\
\quad+(-1)^{\left|a_{0}\right|}\left(\sum(-1)^{\left|a_{1}\right|+\cdots+\left|a_{i-1}\right|+i} a_{0}\left[a_{1}|\cdots| \partial a_{i}|\cdots| a_{\imath}\right]\right. \\
\left.\quad+\sum(-1)^{\left|a_{1}\right|+\cdots+\left|a_{i}\right|+i} a_{0}\left[a_{1}|\cdots| a_{i} a_{i+1}|\cdots| a_{\imath}\right]\right)
\end{aligned}
$$

Let $A$ be a d.g.a. algebra. We say that a multiplicity $\mu$ is defined on $A$, if we have a decomposition of $A$ into a direct sum of the submodules ${ }_{l} A$ indexed by nonnegative integers $l$ such that ${ }_{l} A=\sum_{p=0}^{\infty}{ }_{l} A_{p}$ with ${ }_{l} A_{p}={ }_{l} A \cap A_{p}$, ${ }_{l} A_{m} A \subset{ }_{l+m} A, \partial\left({ }_{l} A\right) \subset{ }_{l} A$ and $\varepsilon \mid{ }_{l} A_{p}=0$ for $l>0$ or $p>0$. An element $a$ of ${ }_{l} A$ is called a homogeneous element of multiplicity $\mu(a)=l$. Let $a$ be an element of $A$. Then we define a multiplicity $\mu(a)$ of $a$ to be the maximum of $l$ such that $a$ has a nonzero component in the direct summand ${ }_{l} A$ of $A$. Now all the elements of $A$ with multiplicity $\leqq n$ generate a d.g.a. submodule of $A$ which will 
be denote by $A(n)$. A d.g.a. algebra $A$ with multiplicity is called free if each component ${ }_{l} A_{p}$ is free.

If a multiplicity $\mu$ is defined on $A$, then $\mu$ is extended to a multiplicity $\tilde{\mu}$ on $B A$ by putting $\tilde{\mu}\left(a\left[a_{1}|\cdots| a_{t}\right]\right)=\mu(a)+\sum_{i=1} \mu\left(a_{i}\right)$. Here we note that we can define two functions on $B A, \mu_{F}$ called the fibre multiplicity and $\mu_{B}$ called the base multiplicity defined by $\mu_{F}\left(a\left[a_{1}|\cdots| a_{\imath}\right]\right)=\mu a$ and $\mu_{B}\left(a\left[a_{1}|\cdots| a_{\imath}\right]\right)=$ $\mu\left(\left[a_{1}|\cdots| a_{t}\right]\right)$ respectively.

A d.g.a. homomorphism $f: A \rightarrow A^{\prime}$ between two d.g.a. algebras $A$ and $A^{\prime}$ with multiplicities $\mu$ is supposed to obey the rule $\mu f=\mu$. Now $f$ can be extended to a d.g.a. homomorphism $\tilde{f}: B A \rightarrow B A^{\prime}$ compatible with $f$ and we have $\bar{f}: \bar{B} A \rightarrow \bar{B} A^{\prime}$. Clearly the multiplicities of $A$ and $A^{\prime}$ define a multiplicity on $A \otimes A^{\prime}$ in a natural way. Then a multiplication $m: A \otimes A \rightarrow A$ is multiplicity preserving and hence it can be extended to the multiplication $\tilde{m}: B A \otimes B A \rightarrow B A$ which gives $B A$ a structure of a d.g.a. algebra with multiplicity. As easily seen, $A$ is a subalgebra of $B A$, so $\bar{B} A=B A / I(A) B A$ is again a d.g.a. algebra with multiplicity.

An explicit formula of $\tilde{m}$ is given as follows: Take the elements

$$
a_{0}\left[a_{1}|\cdots| a_{\imath}\right], \quad b_{0}\left[b_{1}|\cdots| b_{\kappa}\right] .
$$

Let us define the elements $c_{j}$ by

$$
c_{j}=\left\{\begin{array}{l}
a_{j} \\
\beta_{j-1}
\end{array}\right.
$$

for $1 \leqq j \leqq c$ for $\iota+1 \leqq j \leqq \imath+k$.

Let $\pi$ be a permutation

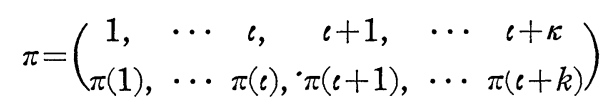

satisfying the relation

$$
\begin{gathered}
\pi(1)<\cdots<\pi(\iota) \\
\pi(\iota+1)<\cdots<\pi(\iota+\kappa) .
\end{gathered}
$$

Now the product is defined by

$$
\begin{aligned}
& \left(a_{0}\left[a_{1}|\cdots| a_{\iota}\right]\right)\left(b_{0}\left[b_{1}|\cdots| b_{\kappa}\right]\right) \\
& \quad=\sum_{\pi} \varepsilon(\pi) a_{0} b_{0}\left[c_{\pi^{-1}(1)}|\cdots| c_{\pi^{-1}(\iota+\kappa)}\right]
\end{aligned}
$$

where $\varepsilon(\pi)= \pm 1$ is given by

$$
\varepsilon(\pi)=(-1)^{\sum_{i=1}^{\ell}\left(\left|a_{i}\right|+1\right)\left|b_{0}\right|+} \sum_{1 \leqq \pi(\iota+k)<\pi(i)}\left(\left|a_{i}\right|+1\right)\left(\left|b_{k}\right|+1\right)
$$

Let $G_{m}$ be the submodule of $B A$ generated by the elements whose base multiplicity $\mu_{B} \leqq m$. Then the family of $G_{m}$ defines a filtration on $B A$. We denote $G_{n-m, m}={ }_{n} B(A) \cap G_{m}$. $\quad E_{l, m}^{0}(G)$ will mean $G_{l, m} / G_{l+1, m-1}$.

Proposition 1.10. Suppose ${ }_{0} A=\Lambda$. Then we have

$$
E_{l, m}^{0}(G) \cong{ }_{l} A \otimes_{m} \bar{B} A
$$

Proof. Take an element $a$ of $\bar{A} . \mu\left(a a_{1}\right)=\mu(a)$ if and only if $\mu\left(a_{1}\right)=0$. From the assumption, this is equivalent to say $a_{1}=0$. Hence we have $a a_{1}=0$ except 
in case $\mu\left(a a_{1}\right)>\mu(a)$. Let $a\left[a_{1}|\cdots| a_{\imath}\right]$ be an element of $G_{l, m}$. Then we have

$$
\begin{aligned}
\partial\left(a\left[a_{1}|\cdots| a_{\imath}\right]\right)= & \partial a\left[a_{1}|\cdots| a_{\imath}\right] \\
& +a a_{1}\left[a_{2}|\cdots| a_{\imath}\right] \\
& +(-1)^{|a|}\left(\sum(-1)^{\left|a_{1}\right|+\cdots+\left|a_{i-1}\right|+i} a\left[a_{1}|\cdots| \partial a_{i}|\cdots| a_{\imath}\right]\right) \\
& \left.\quad+\sum(-1)^{\left|a_{1}\right|+\cdots+\left|a_{i}\right|+i} a\left[a_{1}|\cdots| a_{i} a_{i+1}|\cdots| a_{\imath}\right]\right) \\
\equiv & \partial a\left[a_{1}|\cdots| a_{\imath}\right]+(-1)^{|a|} a \cdot \tilde{\omega} \partial\left[a_{1}|\cdots| a_{\imath}\right] \quad \bmod G_{l+1, m-1} .
\end{aligned}
$$

This concludes the proof.

Let $I$ be an abelian semi-group. Then the d.g.a. algebra $A(\Pi, a)$ is defined inductively as follows: For $q=0, A(\Pi, 0)=\Lambda(\Pi)$ and for $q \geqq 1 \quad A(\Pi, q)=$ $\bar{B} A(\Pi, q-1)$.

In particular, we consider the case $\Pi=N$. Then the multiplicity $\mu$ is defined on $A(N, 0)=\Lambda(N)$ by the rule $\mu\left(\alpha^{l}\right)=l$ and consequently ${ }_{l} A(N, 0)$ is defined to be $\Lambda\left((\alpha-e)^{l}\right)$. To simplify the notation, we write $A(N, q)(n)$ as $A(N, q, n)$ and $B A(N, q)(n)=B(N, q, n)$ in the sequel.

Replacing $A$ by $A(N, q)$ in the previous Proposition, we have

Proposition 1.11.

$$
\begin{aligned}
E_{l, m}^{0}(G) & \cong{ }_{l} A(N, q) \otimes_{m} A(N, q+1) \\
& \cong A(N, q, l) / A(N, q, l-1) \otimes A(N, q+1, m) / A(N, q+1, m-1) .
\end{aligned}
$$

In the rest of this section, we restrict ourselves to the case $\Lambda=Z$.

Proposition 1.12. The natural injection $i: N \rightarrow Z$ induces a chain equivalence

$$
A(i, q): A(N, q) \rightarrow A(Z, q)
$$

Proof. We only need to verify the proposition in the case $q=1$. For this purpose, we compare two constructions $C(N)$ and $C(Z)$ defined as follows.

$\mathrm{C}(Z)$ is a twisted tensor product $\Lambda(Z) \otimes E(x)$ with the boundary operator defined by $\partial\left(\alpha^{l} \otimes x\right)=\left(\alpha^{l}-\alpha^{l+1}\right) \otimes 1$ and $C(N)$ is its subalgebra $\Lambda(N) \otimes E(x)$. Clearly $A(i, 1)$ induces an isomorphism $i d: E(x) \rightarrow E(x)$. q.e.d.

\section{§. Equivalence between $K(N, q, n)$ and $A(N, q, n)$.}

Throught this section, $K$ denotes a unitary $F D$-algebra with multiplicity and $N K$ the normalized $F D$-module of $K(\mathrm{Cf}$. Eilenberg [9], Dold [5]) $W K$ denotes the $W$-construction of $K$. Furthermore $A$ denotes a unitary d.g.a. algebra withmultiplicity and $K A$ the $F D$-module derived from $A$ (Cf. Dold [5]), $B A$ the barconstruction of $A$.

Proposition 1.13. We can define a FD-homomorphism $\varphi: W K A \rightarrow K B A$ which satisfies the conditions : $\varphi \mid K A=i d$ and $\mu \varphi=\mu$.

Proof. Define a homomorphism $\tilde{\varphi}: W K A \rightarrow K B A$ by the formula

$$
\tilde{\varphi}(\sigma \times \tau)=\left\{\begin{array}{lr}
s \tilde{\varphi} \partial(1 \times \tau) & \text { if } \sigma=1 \\
\sum \partial_{u+1}^{v} \sigma \cdot \tilde{\varphi} \partial_{0}^{u}(1 \times \tau) & \text { otherwise }
\end{array}\right.
$$

As easily seen, $\tilde{\varphi}$ induces a homomorphism $\varphi: W K A \rightarrow K B A$ and satisfies the condition $\varphi \mid K A=i d$. We shall verify $\mu \varphi=\mu$.

Let $\partial \times \tau$ be a $(p+1)$-simplex of $W K A$ not belonging to $K A \times(\bar{W} K A)^{p}$. If $\sigma=1$,

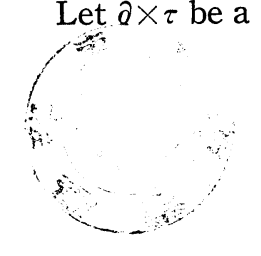


then $\tilde{\varphi}(1 \times \tau)=s \tilde{\varphi} \partial(1 \times \tau)$. Since we know that $\mu s=\mu$ and $\mu \partial=\mu$, we have $\mu \tilde{\varphi}(1 \times \tau)=$ $\mu(1 \times \tau)$. In case $\sigma \neq 1, \mu \tilde{\varphi}=\mu$ is proved as follows :

$$
\begin{aligned}
\mu \tilde{\varphi}(\sigma \times \tau) & =\mu \sum \partial_{u+1}^{v} \sigma \cdot \tilde{\varphi} \partial_{0}^{u}(1 \times \tau) \\
& =\max \mu\left(\partial_{u+1}^{v} \sigma \cdot \tilde{\varphi} \partial_{0}^{u}(1 \times \tau)\right) \\
& =\max \mu\left(\left(\partial_{u+1}^{v}(\sigma \times 1) \cdot \tilde{\varphi} \partial_{0}^{n}(1 \times \tau)\right)\right. \\
& =\max \mu\left(\tilde{\varphi} \partial_{u+1}^{v}(\sigma \times 1) \cdot \tilde{\varphi} \partial^{u}(1 \times \tau)\right) \\
& =\mu(\sigma \times 1)+\mu(1 \times \tau)
\end{aligned}
$$

where max means the maximum taken over the set of couples $(u, v)$ satisfying $\partial_{u+1}^{v} \sigma \neq 0$ and $\tilde{\varphi} \partial_{0}^{u}(1 \times \tau) \neq 0$. These results lead to the conclusion

$$
\mu \tilde{\varphi}(\sigma \times \tau)=\mu(\sigma \times \tau) .
$$

Corollary 1.14. There exists a FD-homomorphism

$$
\bar{\varphi}: \bar{W} K A \rightarrow K \bar{B} A
$$

satisfying $\mu \bar{\varphi}=\mu$.

Conversely, we have the following proposition.

Proposition 1.15. We can define a d.g.a. homomorphism $\psi: B N K \rightarrow N W K$ which satisfies the conditions: $\psi \mid N K=i d$. and $\mu \psi=\mu$.

Proof. Define a homomorphism $\tilde{\phi}: B K \rightarrow W K$

$$
\tilde{\phi}(\sigma \otimes \tau)=\left\{\begin{array}{lr}
s \tilde{\psi} \partial(1 \otimes \tau) & \text { if } \sigma=1 \\
\sum \varepsilon s_{j_{v}} \cdots s_{j_{1}} \sigma \cdot s_{i_{u}} \cdots s_{i_{1}} \tilde{\psi}(1 \otimes \tau) & \text { otherwise },
\end{array}\right.
$$

where $\varepsilon$ denotes an appropriate signature \pm 1 . $\tilde{\psi}$ induces $\phi: B N K \rightarrow N W K$. We shall prove $\mu \tilde{\psi}=\mu$.

Now we denote $N K$ by $A$. Let $\sigma \otimes \tau$ be a $(p+1)$-element of $B A$ not belonging to $A \otimes(\bar{B} A)^{p}$. If $\sigma=1$, then $\tilde{\psi}(1 \otimes \tau)=s \tilde{\psi} \partial(1 \otimes \tau)$. Hence we have $\mu \tilde{\psi}(1 \otimes \tau)=$ $\mu(1 \otimes \tau)$. In case $\sigma \neq 1, \mu \psi=\mu, \mu \tilde{\psi}=\mu$ is proved as before, that is :

$$
\begin{aligned}
\mu \tilde{\psi}(\sigma \otimes \tau) & =\mu \sum \varepsilon\left(s_{j_{v}} \cdots s_{j_{1}} \sigma \cdot s_{i_{u}} \cdots s_{i_{1}} \tilde{\psi}(1 \otimes \tau)\right) \\
& =\max \mu\left(\left(s_{j_{v}} \cdots s_{j_{1}} \sigma \times 1\right) \cdot s_{i_{u}} \cdots s_{i_{1}} \widetilde{\psi}(1 \otimes \tau)\right) \\
& =\max \mu\left(s_{j_{v}} \cdots s_{j_{1}} \tilde{\psi}(\sigma \otimes 1) \cdot s_{i_{u}} \cdots s_{i_{1}} \tilde{\psi}(1 \otimes \tau)\right) \\
& =\mu(\sigma \otimes \tau) .
\end{aligned}
$$

Corollary 1.16. There exists a d.g.a. homomorphism

$$
\bar{\psi}: \bar{B} N K \rightarrow N \bar{W} K
$$

satisfying $\mu \bar{\psi}=\mu$.

In the following, chain homotopies are assumed to be multiplicity preserving.

Proposition 1.17. Let $K$ and $K^{\prime}$ be two unitary FD-algebras with multiplicity. Suppose given two FD-homomorphisms $f_{0}, f_{1}: K \rightarrow K^{\prime}$ and an FD-homotopy $h: K \times \Delta(1) \rightarrow K^{\prime}$ such that $\partial h+h \partial=f_{1}-f_{0}$. Then $h$ can be extended to an $F D$ hhomotory $\tilde{h}: W K \times \Delta(1) \rightarrow W K^{\prime}$ such that $\partial \tilde{h}+\tilde{h} \partial=\tilde{f_{1}}-\tilde{f_{0}}$ where $\tilde{f}_{i}$ denotes an extension of $f_{i}$. Consequently $\tilde{h}$ defines an FD-homotopy $\bar{h}: \bar{W} K \times \Delta(1) \rightarrow W K^{\prime}$ such that $\partial \bar{h}+\bar{h} \partial=\bar{f}_{1}-\bar{f}_{0}$.

Proposition 1.18. Let $A$ and $A^{\prime}$ be two d.g.a. algebras with mulniplicity. 
Suppose given two d.g.a. homomorphisms $f_{0}, f_{1}: A \rightarrow A^{\prime}$ and a chain homotopy $h: A \rightarrow A^{\prime}$ such that $\partial h+h \partial=f_{1}-f_{0}$. Then $h$ can be extended to a chain homotopy $\tilde{h}: B A \rightarrow B A^{\prime}$ such that $\partial \tilde{h}+\tilde{h} \partial=\tilde{f}_{1}-\tilde{f_{0}}$ where $\tilde{f}_{i}$ denotes an extension of $f_{i}$. Consequently $\tilde{h}$ defines a chain homotopy $\bar{h}: \bar{B} A \rightarrow \bar{B} A^{\prime}$ such that $\partial \bar{h}+\bar{h} \partial=\bar{f}_{1}-\bar{f}_{0}$.

Combining these results, we have

THeOREм 1.19. We have chain equivalences

$$
\begin{aligned}
& \varphi=\varphi(q): K(N, q) \rightarrow A(N, q) \\
& \psi=\psi(q): A(N, q) \rightarrow K(N, q)
\end{aligned}
$$

such that $\mu \varphi=\mu$ and $\mu \psi=\mu$. These equivalences induces equivalences

$$
\begin{aligned}
& \varphi(q, n): K(N, q, n) \rightarrow A(N, q, n) \\
& \psi(q, n): A(N, q, n) \rightarrow K(N, q, n)
\end{aligned}
$$

As a consequence of Proposition 1.12 and Theorem 1.19, we have

Corollary 1.20. The natural injection $i: N \rightarrow Z$ induces a chain equivalence

$$
K(i, q): K(N, q) \rightarrow K(Z, q) \text {. }
$$

\section{$\S 4$. Supplementary results}

1. $K_{\theta}(N, q)$

Given an $F D$-homomorphism $T: K \rightarrow K^{\prime}$ between unitary $F D$-algebras $K$ and $K^{\prime}$, we define an $F D$-module $K^{\prime} \underset{\bar{T}}{\times} \bar{W} K$ over $K^{\prime}$ as follows :

The $p$-chain module is given by

$$
\left(K^{\prime} \underset{\bar{T}}{\times} \bar{W} K\right)_{p}=\left(K^{\prime} \times \bar{W} K\right)_{p}
$$

If we denote by $\sigma \times \tau$ the element of $\left(K^{\prime} \times \overline{\bar{T}} \times \bar{W} K\right)_{p}$ corresponding to the tensor product $\sigma \otimes \tau$ with $\sigma \in K_{p}^{\prime}$ and $\tau \in(\bar{W} K)_{p}$, the face operators $\partial_{i}$ and the degeneracy operators $s_{i}$ are defined by

$$
\begin{aligned}
& \partial_{0}(\sigma \times \tau)=\partial_{0}(\sigma) \cdot t(\tau) \times \partial_{0} \tau \\
& \partial_{i}(\sigma \times \tau)=\partial_{i} \sigma \times \partial_{i} \tau \\
& s_{i}(\sigma \times \tau)=s_{i} \sigma \times s_{i} \tau
\end{aligned}
$$
for $i \geqq 0$

where $t:(\bar{W} K)_{p} \rightarrow K_{p-1}^{\prime}$ denotes a functional given by $t\left\langle\sigma_{p-1}, \cdots, \sigma_{0}\right\rangle=T\left(\sigma_{p-1}\right)$. Obviously $K \times \bar{W} K=W K$. We remark here the existence of an $F D$-homomorphism $i d \times \bar{T}^{i d}: K^{\prime} \times \bar{T} \times \bar{W} K \rightarrow W K^{\prime}$ defined by $(i d \times \bar{T})(\sigma \times \tau)=\sigma \times \bar{T}(\tau)$ with the induced FD-homomorphism $\bar{T}: \bar{W} K \rightarrow \bar{W} K^{\prime}$.

Let $K$ and $K^{\prime}$ be unitary $F D$-algebras with multiplicities. Let $T: K \rightarrow K^{\prime}$ be an $F D$-homomorphism preserving multiplicity. Then we can define a multiplicity $\mu$ pn $K^{\prime} \times \bar{W} K$ by the formula $\mu(\sigma \times \tau)=\mu(\sigma)+\mu(\tau)$. Furthermore the fibre multiplicity $\mu_{F}$ and the base multiplicity $\mu_{B}$ are defined by $\mu_{F}(\rho \times \tau)=\mu(\sigma)$ and $\mu_{B}(\sigma \times \tau)=\mu(\tau)$ respectively. Let $F_{m}$ be the $F D$-submodule of $K^{\prime} \times \bar{W} K$ generated by the elements $\mu_{B} \leqq m$. Put $F_{n-m, m}={ }_{n}\left(K^{\prime} \times \underset{T}{T} K\right) \cap F_{m}$ and $E_{l, m}^{0} \bar{T}(F)=F_{l, m} / F_{l+1, m-1}$. Anologously to Proposition 1.1, we have

Proposition 1.21.

$$
E_{l, m}^{0}(F) \cong_{l} K^{\prime} \times{ }_{m} \bar{W} K
$$


Len $K$ and $K^{\prime}$ be two unitary $F D$-algebras with multiplicities. Now an $F D$-homomorphism $T_{\theta}: K \rightarrow K^{\prime}$ is defined by $T_{\theta}(\sigma)=\theta^{l} \sigma$ for $\sigma \in{ }_{l} K$ where $\theta$ denotes a nonnegative integer. Let $\bar{T}_{\theta}: \bar{W} K \rightarrow \bar{W} K^{\prime}$ be the $F D$-homomorphism induced by $T_{\theta}$. Then easy compunation shows $\bar{T}_{\theta}=T_{\theta}$.

Now we consider the case $K=K(N, q)$. The homomorphism $\theta: N \rightarrow N$ defined by $\theta(\alpha)=\alpha^{\theta}$ induces an $F D$-homomorphism $K(\theta, q): K(N, q) \rightarrow K(N, q)$. Now we can prove than $T_{\theta}$ is equivalent to $K(\theta, q)$ for $q \geqq 1$. If $q=1, K(N, 1)$ is equivalent to an $F D$-submodule $K(N, 1,1)$ on which two $F D$-homomorphisms $T_{\theta}$ and $K(\theta, q)$ are equivalent to each other. For $q>0$, the equivalence is proved by induction. Well-known argument shows that

$$
K(N, q) \underset{T_{\theta}}{\times} K(N, q+1) \simeq K(N, q) \underset{\boldsymbol{K}(\boldsymbol{\theta}, q+1)}{\times} K(N, q+1)
$$

On the other hand, we have another equivalence

$$
K(N, q) \underset{\boldsymbol{K}(\theta)}{\times} K(N, q+1) \simeq K\left(Z_{\theta}, q\right)
$$

For the sake of simplicity, we introduce here the abbreviation $K_{\theta}(N, q)$ for $K(N, q) \underset{T_{\theta}}{\times} K(N, q+1)$. Then we have obviously

Proposition 1.22 .

$$
\begin{aligned}
& K_{1}(N, q) \cong W(N, q) \\
& K_{\theta}(N, q) \simeq K\left(Z_{\theta}, q\right)
\end{aligned}
$$$$
\text { for } \theta \geqq 1 \text {. }
$$

From the above discussion, a multiplicity $\mu$ is defined on $K_{\theta}(N, q) . K_{\theta}(N, q, n)$ denotes $K_{\theta}(N, q)(n)$. Now we have clearly

Proposition 1.23.

$$
E_{l, m}^{0}(F) \cong{ }_{l} K(N, q) \times{ }_{m} K(N, q+1) .
$$

Moreover we have an anologue of Proposition 1.9.

Proposition 1.24 .

$$
\begin{aligned}
K_{\theta}(N, q, 1) & \simeq K\left(X_{\theta}^{q}\right) \\
K_{\theta}(N, q, n) & \simeq K\left(\operatorname{SP}^{n} X_{\theta}^{q}\right) \\
{ }_{1} K_{\theta}(N, q) & =M\left(Z_{\theta}, q\right)
\end{aligned}
$$

where $X_{\theta}^{q}$ means a space $S_{\theta}^{q} \cup_{\theta}^{q+1}$ obtained by attaching $a(q+1)$-cell to a $q$-sphere on its boundary with degree $\theta$.

2. $A_{\theta}(N, q)$

Given a d.g.a. homomorphism $T: A \rightarrow A^{\prime}$ between d.g.a. algebras $A$ and $A^{\prime}$, we define a d.g.a module $A^{\prime} \underset{\bar{T}}{\otimes} \bar{B} A$ over $A^{\prime}$ as follows :

The $p$-chain module is given by

$$
\left(A^{\prime} \otimes \bar{B} A\right)_{p}=\left(A^{\prime} \otimes \bar{B} A\right)_{p}
$$
If we take an element $a \otimes b$ of $A^{\prime} \frac{\otimes}{T} \bar{B} A$ with $a \in A^{\prime}$ and $b \in \bar{B} A$, the boundary
operator is defined by

$$
\begin{aligned}
& \partial(a \otimes b)=\partial a(1 \otimes b)+(-1)^{|a|} a \cdot \partial(1 \otimes b) \\
& \partial(1 \otimes b)=1 \otimes \partial b+\varphi \cap b
\end{aligned}
$$


where $\varphi:(\bar{B} A)_{p} \rightarrow A_{p-1}^{\prime}$ denotes a function defined by $\varphi\left[a_{1}|\cdots| a_{\imath}\right]=T\left(a_{1}\right)$ if $\iota=1$ and otherwise zero. The cap product notation $\varphi \cap b$ means an element of the form $T\left(a_{1}\right) \otimes\left[a_{2}|\cdots| a_{\imath}\right]$ for $b=\left[a_{1}|\cdots| a_{\imath}\right]$. Clearly we have $A \otimes \bar{B} A=B A$. We remark here the existence of a d.g.a. homomorphism id $\otimes \bar{T}^{i d}: A^{\prime} \otimes_{T} \bar{B} A \rightarrow B A^{\prime}$ obtained by setting $(i d \otimes \bar{T})(a \otimes b)=a \otimes \bar{T}(b)$ with the induced homomorphism $\bar{T}: \bar{B} A \rightarrow \bar{B} A^{\prime}$.

Let $A$ and $A^{\prime}$ be two d.g.a algebras with multiplicities. Let $T: A \rightarrow A^{\prime}$ be a d.g.a. homomorphism preserving multiplicity. Then we can define a multiplicity $\mu$ on $A^{\prime} \otimes_{T} \bar{B} A$ by the formul $\mu(a \otimes b)=\mu(a)+\mu(b)$. Furthermore the fibre multiplicity $\mu_{F}$ and the base multiplicity $\mu_{B}$ are defined by $\mu_{F}(a \otimes b)=\mu(a)$ and $\mu_{B}(a \otimes b)=\mu(b)$ respectively. Let $G_{m}$ be the d.g.a. submodule of $A^{\prime} \otimes_{T} \bar{B} A$ generated by the elements of $\mu_{B} \leqq m$. Put $G_{n-m, m}={ }_{n}\left(A^{\prime} \underset{T}{\otimes} \bar{B} A\right) \cap G_{m}$ and $E_{l, m}^{0}(G)=$ $G_{l, m} / G_{l+1, m-1}$. Cleary we have

Proposition 1.25.

$$
E_{l, m}^{0}(G) \cong_{l} A \otimes_{m} \bar{B} A .
$$

Let $A$ and $A^{\prime}$ be two d.g.a. algebras with multiplicities. Now a d.g.a. homomorphism $T_{\theta}: A \rightarrow A^{\prime}$ is defined by $T_{\theta}(a)=\theta^{l} a$ for $a \in_{l} A$ where $\theta$ denotes a nonnegative integer. Let $\bar{T}_{\theta}: \bar{B} A \rightarrow \bar{B} A^{\prime}$ be the d.g.a. homorphism induced by $T_{\theta}$. Then easy computation shows $\bar{T}_{\theta}=T_{\theta}$.

Now we consider the case $A=A(N, q)$. The homomorphism $\theta: N \rightarrow N$ defined by $\theta(\alpha)=\alpha^{\theta}$ induces a d.g.a. homomorphism $A(\theta, q): A(N, q) \rightarrow A(N, q)$. Now we can prove that $T_{\theta}$ is equivalent to $A(\theta, q)$ for $q \geqq 1$. We notice the equivalence

$$
A(N, q) \underset{T_{\theta}^{\prime}}{\otimes} A(N, q+1) \simeq A(N, q) \underset{A(\theta, q+1)}{\otimes} A(N, q+1)
$$

and another one

$$
A(N, q) \bigotimes_{A(\theta q+1)} A(N, q+1) \simeq A\left(Z_{\theta}, q\right) .
$$

For the sake of simplicity, we introduce the notation $A_{\theta}(N, q)$ instead of $A(N, q) \underset{T_{\theta}}{\otimes} A(N, q+1)$. Then we have

Proposition 1.26 .

$$
\begin{aligned}
& A_{1}(N, q) \cong B(N, q) \\
& A_{\theta}(N, q) \simeq A\left(Z_{\theta}, q\right) \quad \text { for } \theta \geqq 1 .
\end{aligned}
$$

Obviously a multiplicity $\mu$ is defined on $A_{\theta}(N, q) . \quad A_{\theta}(N, q, n)$ denotes $A_{\theta}(N, q)(n)$. Now we have

Proposition 1.27.

3. $C\left(Z_{\theta}, q\right)$

A cochain module $C$ means a module which is a direct sum of the submodules $C^{p}$ indexed by integers $p$ with the coboundary operator $\delta: C^{p} \rightarrow C^{p+1}$ such that $\delta\left(C^{p}\right) \subset C^{p+1}$ and $\delta \delta=0$. A cochain homomorphism $f: C \rightarrow C^{\prime}$ between two cochain modules $C$ and $C^{\prime}$ is a homomorphism such that $f\left(C^{p}\right) \subset C^{p}$ and $\delta f=f \delta$. Here we remark that any chain module $M$ can be regarded as a cochain module by setting $M^{-p}=M_{p}$. 
Let $A$ be an associative algebra over $\Lambda$. A cochain module $C$ over $A$ means a cochain module on which a multiplication $A \otimes C \rightarrow C$ of $A$ from the left is defined in such a way that $A \cdot C^{p} \subset C^{p}$ and $\left(a \cdot a^{\prime}\right) c=a \cdot\left(a^{\prime} \cdot c\right)$ and $\delta(a \cdot c)=a \cdot \delta \cdot c$. Let $C$ and $C^{\prime}$ be two cochain modules over $A$ and $A^{\prime}$ respectively. Suppose given a homomorphism $f: A \rightarrow A^{\prime}$. Cochain homomorphism $\tilde{f}: C \rightarrow C^{\prime}$ compatible with $f$ is a cochain homomorphism such that $\tilde{f(a} \cdot c)=f(a) \cdot \tilde{f}(c)$ for $a \in A$ and $c \in C$. In case $A$ is a group ring $\Lambda(\Pi)$ of $\Pi, A$ will be abbreviated simply as $\Pi$.

A cochain module $C$ with a multiplicity $\mu$ is a cochain module which is a direct sum $\sum_{l} C$ of cochain submodules ${ }_{l} C$ indexed by non-negative integers $l$. A cochain homomorphism $f: C \rightarrow C^{\prime}$ between two cochain modules is supposed to obey the rule $\mu f=\mu$.

In the sequel, $W(n)$ means a system of projective resolutions of $Z$ over the group ring $Z(S(n))$ which constituts a direct system with injections $i_{m}^{n}$ : $W(m) \rightarrow W(n)$ compatibje with the natural injection $S(m) \rightarrow S(n)$ for $m<n$ satisfying the relation $i_{m}^{n} i_{l}^{m}=i_{l}^{u}$ for $l<m<n$. Suppose given a cochain module $C$ such that $C_{0} \cong \Lambda$. Then we have a driect system of cochain modules $W(n) \otimes(\otimes C)$ over $S(n)$ and the injections $i_{m}^{n}: W(m) \otimes\left(\otimes_{m} C\right) \rightarrow W(n) \otimes\left(\otimes_{n} C\right)$ compatible with the injection $S(m) \rightarrow S(n)$ defined for $m<n$. Thus we can construct a direct limit $\lim _{n \rightarrow \infty} W(n) \otimes(\otimes C)$ which will be denoted by $W(\infty) \otimes(\otimes C)$. As usual we denote by $W(n) \underset{S(n)}{\otimes}\left(\otimes_{n} C\right)$ the factor module $W(n) \otimes\left(\otimes_{n} C\right) / S(n) . \quad W(\infty) \otimes_{S(\infty)}\left(\otimes_{\infty} C\right)$ means the direct limit $\lim _{n \rightarrow \infty} W(n) \bigotimes_{S(n)}(\underset{n}{\otimes C}$ ). Similar abbreviation will be used in other cases, for instance $S(\infty) \stackrel{n \rightarrow \infty}{=} \lim ^{n} S(n)$.

Now we take the dual $\stackrel{n \rightarrow \infty}{M}\left(Z_{\theta}, q\right)_{N}^{*}$ of $M\left(Z_{\theta}, q\right)_{N}$. If $\theta>0, M\left(Z_{\theta}, q\right)_{N}^{*}$ is a free cochain module with the duals $\sigma^{*}$ and $\tau^{*}$ of the simplices $\sigma$ and $\tau$ in $M\left(Z_{\theta}, q\right)_{N}$ as the free generators in dimensions $q$ and $q+1$ respectively, and with the coboundary operator defined by $\delta \sigma^{*}=\theta \tau^{*} . M(Z, q)_{N}^{*}$ is a free cochain module with generator $\sigma^{*}$ in dimension $q$. In the following, we shall use the notations $u$ and $v$ for the generators $\sigma^{*}$ and $\tau^{*}$. We consider the d.g.a. module $K_{\theta}(N, q, 1)$. Setting $K_{\theta}(N, q, 1)=L\left(Z_{\theta}, q\right), L\left(Z_{\theta}, q\right)_{N}^{*}$ is identified with $M\left(Z_{\theta}, q\right)_{N}^{*}+\Lambda$. Now an arbitrary element of $W(\infty) \otimes\left(\otimes L\left(Z_{\theta}, q\right)_{N}^{*}\right)$ can be written in the form $\xi \otimes x\left(u^{(l)} \otimes v^{(m)} \otimes 1^{(n-l-m)}\right)$ with $\xi \in W(n)$ and $x \in S(n)$, where $u^{(l)}$ means the $l$-th tensor product of $u$. Thus we can introduce a multiplicity $\mu$ by $\mu(c)=l+m$ for the element $c$ of the form $\mu\left(\xi \otimes x\left(u^{(l)} \otimes v^{(m)} \otimes 1^{(n-l-m)}\right)\right)$. Furthermore we can define the fibre multiplicity $\mu_{F}$ and the base multiplicity $\mu_{B}$ by $\mu_{F}(c)=l$ and $\mu_{B}(c)=m$ respectively. In the same way, $\mu, \mu_{F}$ and $\mu_{B}$ can be defined on $W(\infty) \otimes_{S(\infty)}\left(\otimes_{\infty} L\left(Z_{\theta}, q\right)_{N}^{*}\right)$

Let ${ }^{S(\infty)} C^{\infty}$ be a cochain submodule of ${ }^{\prime} C\left(Z_{\theta}, q\right)=W(\infty) \otimes\left(\otimes L\left(Z_{\theta}, q\right)_{N}^{*}\right)$ generated by the elements of $\mu_{B} \geqq m$. The family of ' $C^{m}$ defines a filtiration. Put ${ }^{\prime} C^{n-m, m}=$ ${ }_{n}^{\prime} C\left(Z_{\theta}, q\right) \cap^{\prime} C^{m}$ and $E_{0}^{l, m}\left({ }^{\prime} C\right)={ }^{\prime} C^{l, m} /{ }^{\prime} C^{l-1}{ }^{m+1}$. Analogously, we define $C^{m}$ to be the cochain submodule of $C\left(Z_{\theta}, q\right)=W(\infty) \bigotimes_{S(\infty)}\left(\otimes_{\infty} L\left(Z_{\theta}, q\right)_{N}^{*}\right)$ generated by the elements of $\mu_{B} \geqq m$. Put $C^{n-m, m}={ }_{n} C\left(Z_{\theta}, q\right) \cap C^{m}$ and $E_{0}^{l \cdot m}(C)=C^{l \cdot m} / C^{l-1, m+1}$.

As easily seen, we obtain

Proposition 1.28.

$$
E_{0}^{l, m}(C) \cong W(\infty) \bigotimes_{S(l) \times S(m)}\left(M(Z, q)_{N}^{*(l)} \otimes M(Z, q+1)_{N}^{*(m)}\right) .
$$


Since $W(\infty) \bigotimes_{S(l) \times S(m)}\left(M(Z, q)_{N}^{*(l)} \otimes M(Z, q+1)_{N}^{*(m)}\right)$ is homotopy equivalent to $\left(W(l) \otimes_{S(l)} M(Z, q)_{N}^{*(l)}\right) \otimes\left(W(m) \bigotimes_{S(m)} M(Z, q+1)_{N}^{*(m)}\right)$, we have

Corollary 1.29 .

$$
E_{0}^{l \cdot m}(C) \simeq{ }_{l} C(Z, q) \otimes_{m} C(Z, q+1) .
$$

\section{$\S 5$. The construction of the homomorphism}

$$
\left.{ }_{n} \psi\left(Z_{\theta}, q\right): \underset{n}{\times} M\left(Z_{\theta}, q\right) / \Pi\right)_{N}^{*} \rightarrow W(\Pi) \underset{I I}{\otimes}\left(\underset{n}{\otimes} M\left(Z_{\theta}, q\right)_{N}^{*}\right) .
$$

1. The construction of ${ }_{n} \psi(Z, q)$.

Throughout this section, $I I$ denotes a subgroup of the symmetric group $S(n)$ of degree $n$. We assume that $I I$ satisfies the condition (A) stated below.

Let $K$ be a free $F D$-module. Then $\Pi$ operates on the $n$-th certesian product $\times K$ of $K$. For a fixed $p$, the set of all $p$-simplices of $\times K$ are divided into the set of $\Pi$-orbits $\Gamma_{i}$. Then we can choose one simplex $\rho_{i}$ in each class $\Gamma_{i}$. Let $\Pi(\rho)$ be an isotropy group of $\Pi$ fixing $\rho$ of $\times K$. Now we choose a representative $x_{i l}$ in each of the left cosets $\Pi / \Pi\left(\rho_{i}\right)^{n}$ of $\Pi$ with respect to $\Pi\left(\rho_{i}\right)$. Then an arbitrary $p$-simplex $\rho$ of $\times K$ can be written uniquely in the form $\rho=x_{i l} \rho_{i}$ or equivalently $\rho^{*}=x_{i} \rho_{i}^{*}$.

Now our assumption is given as follows:

(A) Let $\rho_{i}$ be a face of $x_{j k} \rho_{j}$. Then any of the representatives of the left cosets $\Pi / \Pi\left(\rho_{j}\right)$ can be written in the form $x_{i l} x_{\jmath m}^{\prime}$ where $x_{i l}$ denotes a representative of one of the left cosets $\Pi / \Pi\left(\rho_{i}\right)$ and $x_{j m}^{\prime}$ denotes a representative of one of the left cosets $\Pi / \Pi\left(\rho_{j}\right)$ such that $x_{j m}^{\prime} x_{j k}^{-1} \in \Pi\left(\rho_{i}\right)$.

Remark. The assumption (A) implies $\Pi\left(x_{j k} \rho_{j}\right)=x_{j k} \Pi\left(\rho_{j}\right) x_{j k}^{-1} \subset I I\left(\rho_{i}\right)$. Hence $x_{j m}^{\prime} x_{j k}^{-1} \in \Pi\left(\rho_{i}\right)$ is equivalent to the condition $x_{j m}^{\prime} x_{j k}^{-1}$ runs over the set of left cosets $\Pi\left(\rho_{i}\right) / \Pi\left(x_{j k} \rho_{j}\right)$ and consequently $x_{i l} x_{j m}^{\prime} x_{j k}^{-1}$ runs over the set of the left cosets $\Pi / \Pi\left(x_{j k} \rho_{j}\right)$.

Lemma 1.30. Let $\Pi$ be $S(n)$ itself. Then II satitfies (A).

Proof. We introduce a linear order in the set of all $p$-simplices of $K$. Then an arbitrary $p$-simplex $\rho$ of $\times K$ can be written in the form $x\left(\sigma_{1} \times \cdots \times \sigma_{n}\right)$ with $x \in S(n)$ where $\sigma_{\nu}$ are the simplices of $K$ such that $\sigma_{\nu-1} \geqq \sigma_{\nu}$ for $1<\nu \leqq n$. Hence we can define representatives $\rho_{i}$ to be the simplices of the form $\sigma_{1} \times \cdots \times \sigma_{n}$ such that $\sigma_{\mu-1} \geqq \sigma_{\nu}$. Clearly we have the expression $\Pi\left(\rho_{i}\right)=S\left(n_{1}\right) \times \cdots \times S\left(n_{\iota}\right)$ where $n_{i}$ are positive integers satisfying $n_{1} \cdots+n_{l}=n$. We shall call a shuffle with respect to the subgroup $S\left(n_{1}\right) \times \cdots \times S\left(n_{\imath}\right)$ a permutation

$$
x=\left(\begin{array}{c}
1, \cdots, n \\
x(1), \cdots, x(n)
\end{array}\right)
$$

satisfying the relation

$$
x\left(\sum_{j=1}^{i-1} n_{j}+\lambda\right)<x\left(\sum_{j=1}^{i-1} n_{j}+\lambda+1\right)
$$

for $1 \leqq \lambda<n_{i}$. Suppose given one of the left cosets $\Pi / \Pi\left(\rho_{j}\right)$. Then there exists one and only one shuffle belonging to the given coset. So we shall take the 
shuffle as a representative of the given left coset.

Let $\rho_{j}$ be the representative of an orbit $\Gamma_{j}$ whose isotropy subgroup $\Pi\left(\rho_{j}\right)$ is given by $S\left(n_{1}^{\prime}\right) \times \cdots \times S\left(n_{\kappa}^{\prime}\right)$. Now we assume that $\rho_{i}$ is a face of $x_{j k} \rho_{j}$. Then $\Pi\left(\rho_{i}\right)$ can be expressd in the form $S\left(n_{1}\right) \times \cdots \times S\left(n_{\imath}\right)$ and we have

$$
\sum_{j=1}^{u(i)-1} n_{j}<x_{j k}\left(\sum_{j=1}^{i-1} n_{j}^{\prime}+\lambda\right) \leqq \sum_{j=1}^{u(i)} n_{j}
$$

for $1 \leqq \lambda \leqq n_{i}^{\prime}$ with some index $u(i)$. Henth the product $x_{i l} x_{j m}^{\prime}$ is again a shuffle with respect to $\Pi\left(\rho_{j}\right)$ and runs over the set of all the representatives of $\Pi / \Pi\left(\rho_{j}\right)$ This completes the proof.

Remark. Let $\Pi$ be a $p$-Sylow subgroup $S(n, p)$ of $S(n)$ as in Steenrod [18], Then $\Pi$ satisfies (A).

Now we recall the definition of the Eilenberg-Zilber equivalence $\alpha: \times K \rightarrow \otimes K$ and $\beta: \otimes K \rightarrow \times K$.

${ }^{n}$ We define an ordered set of increasing sequences of integers

$$
j(\nu, 1), \cdots, j(\nu, v(\nu))
$$

with $1 \leqq \nu \leqq n$ for which we have a permutation

$$
\pi=(j(1,1), \cdots, j(1, v(1)), \cdots, j(n, 1), \cdots, j(n, v(n)))
$$

of $(0,1, \cdots, p-1)$. Then we define increasing sequences of integers

$$
i(\nu, 1), \cdots, i(\nu, u(\nu))
$$

to be the complementary sets of

$$
j(\nu, 1), \cdots, j(\nu, v(\nu))
$$

in $(0,1, \cdots, p-1)$.

Now the homomorphism $\beta$ is defined by

$$
\beta\left(\sigma_{1} \otimes \cdots \otimes \sigma_{n}\right)=\sum_{\pi} \varepsilon(\pi) s_{i(1, u(1))} \cdots s_{i(1,1)} \sigma_{1} \times \cdots \times s_{i(n, u(n))} \cdots s_{i(n, 1)} \sigma_{n}
$$

where $\varepsilon(\pi)$ denotes a signature of $\pi$ and $v(\nu)=\left|\sigma_{\nu}\right|$.

On the other hand, the homomorphism $\alpha$ is defined by

$$
\alpha\left(\sigma_{1} \times \cdots \times \sigma_{n}\right)=\sum_{\pi=1} \partial_{i^{\prime}(1,1)} \cdots \partial_{i^{\prime}(1, u(1))} \sigma_{1} \otimes \cdots \otimes \partial_{i^{\prime}(n, 1)} \cdots \partial_{i^{\prime}(n, u(n))} \sigma_{n}
$$

where $\sum_{\pi=1}$ means that the summation is extended over the set of sequences $j$ 's such that $\pi=1$, and $i^{\prime}(\nu, \rho)$ are the indices defined by the set of integers $i(\nu, \rho)$.

Let us denote by $M$ the $F D$-module ${ }_{1} K(N, q) \cong M(Z, q)$ and by $W$ the projective resolution $W(I I)$ of $Z$ over $Z(I)$ with suspension operator $s$. In the following, $K^{*}$ denotes a dual $\operatorname{Hom}(K, Z)$. Let $K$ be a free $F D$-module and $\sigma$ be a simplex of $K$. Then $\sigma^{*}$ denotes the dual of $\sigma$. If $K$ is locally finite, $K^{*}$ is a free cochain module with generators $\sigma^{*}$.

Now we introduce a cochain homomorphism

$$
\psi^{\prime \prime}:(\underset{n}{\times} M)_{N}^{*} \rightarrow W \otimes\left(\underset{n}{\otimes} M_{N}^{*}\right)
$$

as follows: 
Clearly $(\underset{n}{\times} M)_{N}^{*}$ contains no simplices in dimensions $p>n q$, so we begin with the dimension $n q$. As mentioned above, we choose one $n q$-simplex $\rho_{i}$ in each $I I$ orbit $\Gamma_{i}$.

If $q=0,(\underset{n}{\times} M)_{N}$ has only one element which is the $n$-th certesian product $(\alpha-e)^{n}$ of $\alpha-e \in M_{0}$. From now on we assume $q>0$. The isotoropy group $\Pi\left(\rho_{i}\right)$ of $\rho_{i}$ consists of only identity for any representative $\rho_{i}$. Hence, an arbitrary $n q$-simplex $\rho$ of $(\underset{n}{\times} M)_{N}$ can be written uniquely in the form $\rho=x \rho_{i}$ or equivalently $\rho^{*}=x \rho_{i}^{*}$ with $x \in S(n)$. Define $\psi^{\prime \prime}\left(\rho^{*}\right)$ by

$$
\psi^{\prime \prime}\left(\rho^{*}\right)=x\left(\xi \otimes u^{(n)}\right)
$$

where $\xi$ denotes a $Z(\Pi)$-base of $W$ in dimension 0 and $u^{(n)}$ denotes the $n$-th tensor product of $u$.

Now we define $\psi^{\prime \prime}$ inductively, and assume that $\psi^{\prime \prime}\left(\rho^{*}\right)$ have been defined for simlices $\rho^{*}$ of dimensions $\geqq p$. We choose a representative $\rho_{i}$ in each one of the $\Pi$-orbits of $(p-1)$-simplices and the representative $x_{i l}$ of each left coset of $\Pi \bmod \Pi\left(\rho_{i}\right)$. Here we should remark that the isotropy group $\Pi\left(\rho_{i}\right)$ always consists of identity, if $\operatorname{dim} \rho_{i}>(n-1) q$. Now any $(p-1)$-simplex $\rho$ of $(\underset{n}{\times} M)_{N}$ can be witten uniquely as $\rho=x_{i l} \rho_{i}$ or equivalently $\rho^{*}=x_{i l} \rho_{i}^{*}$. We define $\psi^{\prime \prime}\left(\rho^{*}\right)$ by

$$
\psi^{\prime \prime}\left(\rho^{*}\right)=x_{i l} s \psi^{\prime \prime} \delta\left(\rho_{i}^{*}\right) .
$$

Here we use the assumption (A). Then the formula $\delta \phi^{\prime \prime}\left(\rho_{i}^{*}\right)=\phi^{\prime \prime} \delta\left(\rho_{i}^{*}\right)$ implies $\delta \phi^{\prime \prime}\left(x_{i l} \rho_{i}^{*}\right)=\phi^{\prime \prime} \delta\left(x_{i l} \rho_{i}^{*}\right)$. Hence $\psi^{\prime \prime}$ is a cochain homomorphism.

Next we shall define from $\phi^{\prime \prime}$ a $\Pi$-equivariant cochain homomorphism

$$
\phi^{\prime}:(\underset{n}{\times} M)_{N}^{*} \rightarrow W \otimes\left(\underset{n}{\otimes} M_{N}^{*}\right) \otimes Q
$$

where $Q$ denotes the field of rational numbers.

Given a simplex $\rho$, we define

$$
\phi^{\prime}\left(\rho^{*}\right)=(1 / \# \Pi(\rho)) \sum_{y \in \Pi(\rho)} y \phi^{\prime \prime}\left(\rho^{*}\right)
$$

where $\# \Pi(\rho)$ denotes the order of $\Pi(\rho)$. Then it is clear that $\phi^{\prime}$ is $\Pi$-equivariant. We put $\delta \rho_{i}^{*}=\sum \varepsilon_{j m} x_{j m} \rho_{j}^{*}$. Then we have $\Pi\left(x_{j m} \rho_{j}\right) \subset \Pi\left(\rho_{i}\right)$ if $\varepsilon_{j m}= \pm 1$ and

$$
\psi^{\prime}\left(x_{j m} \rho_{j}^{*}\right)=\left(1 / \# P_{j m}\right) \sum_{z \in P_{j m}} z \psi^{\prime \prime}\left(x_{j m} \rho_{j}^{*}\right)
$$

where $P_{j m}$ denotes $\Pi\left(x_{j m} \rho_{j}\right)$. Consequently we have

$$
\begin{aligned}
\phi^{\prime}\left(\delta \rho_{i}^{*}\right) & =\left(1 /{ }^{*} P\right) \sum_{y \in P} y \sum_{j, m}\left(1 / P_{j m}\right) \sum_{z \in P_{j m}} z \varepsilon_{j m} \psi^{\prime \prime}\left(x_{j m} \rho_{j}^{*}\right) \\
& =(1 / \# P) \sum_{y \in P} y \sum_{j, m} \varepsilon_{j m} \psi^{\prime \prime}\left(x_{j m} \rho_{j}^{*}\right) \\
& =(1 / \# P) \sum_{y \in P} y \psi^{\prime \prime}\left(\delta \rho_{i}^{*}\right)
\end{aligned}
$$

where $P$ denotes $\Pi\left(\rho_{i}\right)$. This concludes the proof of

$$
\phi^{\prime}\left(\delta \rho_{i}^{*}\right)=\delta \phi^{\prime}\left(\rho_{i}^{*}\right) .
$$

Thus we have obtained a $\Pi$-equivariant cochain homomorphism $\phi^{\prime}$. 
In view of the isomorphism $\left(\underset{n}{\times}{ }_{1} K(N, q)\right)_{N} / S(n) \cong{ }_{n} K(N, q)_{N}$ and the finite ness of ${ }_{1} K(N, q)$, we have a cochain homomorphism

$$
{ }_{n} \psi(Z, q):{ }_{n} K(N, q)_{N}^{*} \rightarrow W(n) \underset{S(n)}{\otimes}\left(\otimes_{n} M(Z, q)_{N}^{*}\right)
$$

which will be abbreviated as $\phi$.

2. The construction of ${ }_{n} \phi(0, q)$.

By arguments similar to those used in the above construction of $\phi$, we can introduce the cochain homomorphism

$$
\tilde{\psi}={ }_{n} \psi(0, q):{ }_{n} W(N, q)_{N}^{*} \rightarrow W(n) \underset{S(n)}{\otimes}\left(\otimes_{n} M(0, q)_{N}^{*}\right) .
$$

This is defined as follows:

We denote by $\tilde{M}$ the $F D$-module ${ }_{1} W(N, q)=M(0, q)$. Let $\sigma_{q}$ and $\tau_{q+1}$ be the non-degerate simplices of ${ }_{1} K(N, q)$ in dimension $q$ and of ${ }_{1} K(N, q+1)$ in dimension $q+1$ respectively. We put $\rho^{\prime}=\sigma_{q} \times 1$ aud $\tau^{\prime}=1 \times \tau_{q+1}$. Then $\sigma^{\prime}$ and $\tau^{\prime}$ generate $\tilde{M}_{N}$. The duals $\sigma^{\prime *}$ and $\tau^{\prime *}$ of $\sigma^{\prime}$ and $\tau^{\prime}$ are denoted by $u$ and $v$ respectively.

Next we introduce a suspension operator $\tilde{s}$ on $W \otimes\left({ }_{n} \underset{M_{N}^{*}}{*}\right)$. Let $s$ be a suspension on $W$. Let $s_{n}$ be a suspension on $\otimes_{n} \tilde{M}_{N}^{*}$ defined by

$$
s^{n}=s_{1} \otimes i d^{(n-1)}
$$

and

$$
s_{1}(v)=u, \quad s_{1}(u)=0
$$

where $i d^{(n-1)}$ denotes the $(n-1)$-th tensor prodect of the identity. Now we define $\tilde{s}=s \otimes i d+\varepsilon \otimes s^{(n)}$.

Since $(\underset{n}{\times} \tilde{M})_{N}^{*}$ has no simplices in dimensions $p>n(q+1)$, we begin with the dimension $n(q+1)$. We choose a representative $\rho_{i}$ in each $\Pi$-orbit $\Gamma_{i}$. As easily seen, $\beta\left(\tau^{\prime} \otimes \cdots \otimes \tau^{\prime}\right)$ is a linear combination of all $n(q+1)$-simplices of $(\underset{n}{\times} \tilde{M})_{N}$ with coefficients \pm 1 . Hence, an arbitrary $n(q+1)-\operatorname{simplex} \rho$ of $(\underset{n}{\times} \tilde{M})_{N}$ can be written uniquely in the form $\rho=x \rho_{i}$ with $x \in S(n)$. Define $\tilde{\psi}^{\prime \prime}\left(\rho^{*}\right)$ by

$$
\tilde{\phi}^{\prime \prime}\left(\rho^{*}\right)=x\left(\xi \otimes v^{(n)}\right)
$$

where $\xi$ denotes a $Z(\Pi)$-base of $W$ in dimension 0 and $v^{(n)}$ denotes the $n$-th tensor product of $v$.

Then we can construct a homomorphism

$$
\tilde{\phi}^{\prime \prime}:(\underset{n}{\times} M) \underset{N}{*} \rightarrow W \otimes\left(\underset{n}{\otimes} \tilde{M}_{N}^{*}\right)
$$

by induction on the dimension using the same argument as before. According to the assmption (A), $\tilde{\psi}^{\prime \prime}$ commutes with $\delta$ too.

We define a homomorphism

$$
\tilde{\psi}:(\underset{n}{\times} \tilde{M})_{N}^{*} \rightarrow W \otimes\left(\bigotimes_{n} \tilde{M}_{N}^{*}\right) \otimes Q
$$


by the same formula as for $\phi^{\prime}$. Clearly $\tilde{\psi}^{\prime}$ is a $\Pi$-equivariant cochain homomorphism. As easily seen, $\tilde{\psi}^{\prime}$ gives rise to a required homomorphism $\tilde{\psi}=$ ${ }_{n} \psi(0, q)$.

Now, we investigate some properties of $\tilde{\phi}^{\prime \prime}$. In the following, we shall restrict ourselves to the case $\Pi=S(n)$ and we shall use the simplified notations $\Pi^{\prime}, \Pi^{\prime \prime}, M^{\prime}$ and $M^{\prime \prime}$ for $S(l), S(m), M(Z, q)$ and $M(Z, q+1)$ respectively.

First, we introduce some notations.

Let us denote by $\underset{\infty}{\times} W(N, q, 1)$ the direct limit $\lim _{n \rightarrow \infty} \underset{n}{\times} W(N, q, 1)$. Clearly the multiplicy $\mu$ on ${ }^{\infty}(N, q, 1)$ can be extended to $\underset{\infty}{\times} W(N, q, 1)$ and the base multiplicity $\mu_{B}$ and the fibre multiplicity $\mu_{F}$ are also extended to $\times W(N, q, 1)$.

Let ' $F^{m}$ be the cochain submodules of $(\underset{\infty}{\times} W(N, q, 1))_{N}^{*}$ generated by the dual of simplices of $\mu_{B} \geqq m$. The family of ${ }^{\prime} F^{m}$ defines a filtration. Put ${ }^{\prime} F^{n-m, m}=$ ${ }_{n}(\underset{\infty}{\times} W(N, q, 1))_{N}^{*} \cap^{\prime} F^{m}$ and $E_{0}^{l, m}\left({ }^{\prime} F\right)={ }^{\prime} F^{l, m} / F^{l-1, m+1}$.

Then we have

Lemma. 1.31 .

$$
\tilde{\phi}^{\prime \prime}\left({ }^{\prime} F^{l, m}\right) \subset^{\prime} C^{l, m}
$$

More precisely, for a simplex $\rho$ of $\mu_{F}(l)=l$ and $\mu_{B}(\rho)=m$, we have

$$
\tilde{\phi}^{\prime \prime}\left(\rho^{*}\right) \equiv \xi \otimes u^{(l)} \otimes v^{(m)} \quad \bmod { }^{\prime} C^{l-1, m+1} .
$$

In particular, in case $\operatorname{dim} \rho=l q+m(q+1), \xi$ can be taken as a $\Pi$-base of $W$ in dimension 0 .

Rroof. We proceed by induction on the base multiplicities $\mu_{B}$ and the dimensions.

If $m=n$, the proposition trivially holds. Hence we assume the Lemma for the simplices $\rho \in(\underset{n}{\times} \tilde{M})_{N}$ with $\mu_{B}(\rho) \geqq m+1$.

Let us consider a $l q+m(q+1)$-simplex $\rho \in(\underset{n}{\times} \tilde{M})_{N}$ with $\mu_{B}(\rho)=m$. The definition of $\beta$ implies

$$
\begin{aligned}
\beta\left(\sigma^{\prime(l)} \otimes \tau^{\prime(m)}\right)=\sum & \varepsilon s_{i(1, u(1))} \cdots s_{i(1,1)} \sigma^{\prime} \times \cdots \times s_{i(l, u(l))} \cdots s_{i(l, 1)} \sigma^{\prime} \\
& \times s_{j(1, v(1))} \cdots s_{j(1,1)} \tau^{\prime} \times \cdots \times s_{j(m, v(m))} \cdots s_{j(m, 1)} \tau^{\prime}
\end{aligned}
$$

It follows that $\beta\left(\sigma^{\prime(l)} \otimes \tau^{\prime(m)}\right)$ is a linear combination of all nondegenerate $l q+$ $m(q+1)$-simplices $\rho$ of $(\underset{n}{\times} \tilde{M})_{N}$ with $\mu_{B}(\rho)=m$ and coefficients \pm 1 .

We notice here the following identitities

$$
\begin{aligned}
& \partial_{i} s_{i_{u}} \cdots s_{i_{1}} \sigma^{\prime}=\left\{\begin{array}{lr}
s_{i_{u}-1} \cdots s_{i_{\rho+1}-1} s_{i_{\rho-1}} \cdots s_{i_{1}} \sigma^{\prime} & i=i_{\rho} \text { or } i_{\rho}+1 \text { for some } \rho \\
1_{q+u-1} & \text { otherwise }
\end{array}\right. \\
& \partial_{0} s_{i_{u}} \cdots s_{i_{1}} \tau^{\prime}= \begin{cases}s_{i_{u^{-1}}} \cdots s_{i_{2^{-1}}} \tau^{\prime} & \text { if } i_{1}=0 \\
s_{i_{u^{-1}}} \cdots s_{i_{1^{-1}}} \sigma^{\prime} & \text { if } i_{1}>0\end{cases} \\
& \partial_{i} s_{i_{u}} \cdots s_{i_{1}-1} \tau^{\prime}=\left\{\begin{array}{lr}
s_{i_{u^{-1}}} \cdots s_{i_{\rho+1}-1} s_{i_{\rho-1}} \cdots s_{i_{1}} \tau^{\prime} & i=i_{\rho} \text { oy } i_{\rho}+1 \text { for some } \rho \\
1_{q+u-1} & \text { otherwise }
\end{array}\right.
\end{aligned}
$$


Suppose given a $l q+m(q+1)$-simplex $\rho$ of $(\underset{n}{\times} \tilde{M})_{N}$ with $\mu_{B}(\rho)=m$.

$$
\begin{aligned}
& \rho=s_{i(1, u(1))} \cdots s_{i(1,1)} \sigma^{\prime} \times \cdots \times s_{i(l, u(l))} \cdots s_{i(l, 1)} \sigma^{\prime} \\
& \quad \times s_{j(1, v(1))} \cdots s_{j(1,1)} \tau^{\prime} \times \cdots \times s_{j(m, v(m))} \cdots s_{j(m, 1)} \tau^{\prime}
\end{aligned}
$$

Let $\rho^{\prime}$ be a $(l q+m(q+1)+1)$-simplex of $(\underset{n}{\times} \tilde{M})_{N}$. Then the above identities show that

and

$$
\rho^{*}\left(\partial_{i} \rho^{\prime}\right)=0
$$

for $i>0$

if and only if

$$
\rho^{*}\left(\partial_{0} \rho^{\prime}\right) \neq 0
$$

$$
\begin{aligned}
& \rho^{\prime}=s_{i(1, u(1))+1} \cdots s_{i(1,1)+1} s_{0} \sigma^{\prime} \times \cdots \\
& \cdots \times s_{i(\nu, u(\nu))+1} \cdots s_{i(\nu, 1)+1} \tau^{\prime} \times \cdots \\
& \cdots \times s_{i(l, u(l)+1} \cdots s_{i(l, 1)+1} s_{0} \sigma^{\prime} \\
& \times s_{j(1, v(1))+1} \cdots s_{j(1,1)+1} s_{0} \tau^{\prime} \times \cdots \times s_{j(m, v(m))+1} \cdots s_{j(m, 1)+1} s_{0} \tau^{\prime} .
\end{aligned}
$$

Hence the hypothesis of induction yields

$$
\tilde{\psi}^{\prime \prime}\left(\delta \rho^{*}\right) \equiv \sum \xi_{\nu} \otimes u^{(\nu-1)} \otimes v \otimes u^{(l-\nu)} \otimes v^{(m)} \quad \bmod { }^{\prime} C^{l-2, m+2}
$$

where $\xi_{\nu}$ denotes a $\Pi$-base of $W$ in dimension 0 . Consequently we have

$$
\tilde{\phi}^{\prime \prime}\left(\rho^{*}\right)=\tilde{s} \tilde{\phi}^{\prime \prime}\left(\delta \rho^{*}\right) \equiv \sum \tilde{s}\left(\xi_{\nu} \otimes u^{(\nu-1)} \otimes v \otimes u^{(l-\nu)} \otimes v^{(m)}\right) \quad \bmod { }^{\prime} C^{l-1, m+1} .
$$

Since $s u=0$ and $s v=u$, the formula $\tilde{s}=s \otimes 1+\varepsilon \otimes s_{n}$ implies

$$
\tilde{\psi}^{\prime \prime}\left(\rho^{*}\right) \equiv \xi \otimes u^{(l)} \otimes v^{(m)} \quad \bmod { }^{\prime} C^{l-1, m+1}
$$

where $\xi$ is some $\Pi$-base of $W$ in dimension 0 . This proves the Lemma in case $\operatorname{dim} \rho=l q+m(q+1)$.

Now we assume that the Lemma holds for the simplices of dimensions $\geqq p$. Let $\rho$ be a $(p-1)$-simplex. Then the hypothesis of induction allows us to obtain the expression

$$
\tilde{\psi}^{\prime \prime}\left(\delta \rho^{*}\right) \equiv \sum \eta \otimes y\left(u^{(l)} \otimes v^{(m)}\right) \quad \bmod { }^{\prime} C^{l-1, m+1}
$$

where $\eta$ denotes an element of $W$ of positive dimension and $y$ is some element of $I$. The definition of the suspension $\tilde{s}$ implies

$$
\tilde{\phi}^{\prime \prime}\left(\rho_{i}^{*}\right)=s \tilde{\psi}^{\prime \prime}\left(\delta \rho_{i}^{*}\right) \equiv 0 \quad \bmod ^{\prime} C^{l-1, m+1}
$$

for the representatives $\rho_{i}$ in $\Pi$ orbits $\Gamma_{i}$ and we have the required result.

3. The construction of ${ }_{n} \psi\left(Z_{\theta}, q\right)$ for $\theta>0$.

For our purpose, we need to consider the $F D$-homomorphism

$$
i d \times T_{\theta}: K_{\theta}(N, q) \longrightarrow W(N, q)
$$

which will be denoted by $\widetilde{T}_{\theta}$ in the sequel. From the definition follows $\mu \widetilde{T}_{\theta}=$ $\mu, \mu_{F} \widetilde{T}_{\theta}=\mu_{F}$ and $\mu_{B} \widetilde{T}_{\theta}=\mu_{B}$. Hence we have two cochain homomorphisms:

$$
\begin{aligned}
& T_{\times}^{\prime}=\left(\underset{n}{\times} \tilde{T}_{\theta}\right)^{*}: \quad(\underset{n}{\times} M(0, q))_{N}^{*} \longrightarrow\left(\underset{n}{\times} M\left(Z_{\theta}, q\right)\right)_{N}^{*} \\
& T_{\otimes}^{\prime}=i d \otimes\left(\otimes_{n} \widetilde{T}_{\theta}^{*}\right): \quad W(\Pi) \otimes\left(\otimes_{n} M(0, q)_{N}^{*}\right) \longrightarrow W(\Pi) \otimes\left(\otimes_{n} M\left(Z_{\theta}, q\right)_{N}^{*}\right) .
\end{aligned}
$$


Since $\tilde{T}_{\theta}$ is injective, these two homomorphisms are also injective.

Now we observe Lemma 1.31 . Let us assume $\Pi=S(n)$. Then we can prove the existence of the cochain homomorphism

$$
{ }_{n} \psi^{\prime \prime}\left(Z_{\theta}, q\right):\left(\underset{n}{\times} M\left(Z_{\theta}, q\right)\right){ }_{N}^{*} \longrightarrow W(\Pi) \otimes\left(\bigotimes_{n} M\left(Z_{\theta}, q\right)_{N}^{*}\right)
$$

so that the following diagram is commutative

$$
\begin{aligned}
& W(\Pi) \otimes\left(\bigotimes_{n} M(0, q)_{N}^{*}\right) \stackrel{T_{\otimes}^{\prime}}{\longrightarrow} W(\Pi) \otimes\left(\bigotimes_{n} M\left(Z_{\theta}, q\right)_{N}^{*}\right) \\
& { }_{n} \psi^{\prime \prime}(0, q) \uparrow \quad \boldsymbol{T}^{\prime} \quad \uparrow_{n} \psi^{\prime \prime}\left(Z_{\theta}, q\right) \\
& (\underset{n}{\times} M(0, q))_{N}^{*} \stackrel{T_{\times}^{\prime}}{\longrightarrow}\left(\underset{n}{\times} M\left(Z_{\theta}, q\right)\right)_{N}^{*} .
\end{aligned}
$$

Also in this case, our familiar argument can be applied to yield a cochain homomorphism

$$
{ }_{n} \psi\left(Z_{\theta}, q\right): K_{\theta}(N, q)_{N}^{*} \longrightarrow W(\Pi) \underset{I I}{\otimes}\left(\bigotimes_{n} M\left(Z_{\theta}, q\right)_{N}^{*}\right)
$$

and a commutative diagram

$$
\begin{array}{ccc}
W(\Pi) \underset{\Pi}{\otimes}\left({\underset{n}{n}}_{\uparrow} M(0, q)_{N}^{*}\right) \stackrel{T_{\otimes}}{\longrightarrow} W(\Pi) \underset{\Pi}{\otimes}\left(\underset{n}{\otimes} M\left(Z_{\theta}, q\right)_{N}^{*}\right) \\
\left.{ }_{n} \psi(0, q)\right|_{n} \psi\left(Z_{\theta}, q\right) \\
W(N, q)_{N}^{*} & \stackrel{T_{\times}}{\longrightarrow} & K_{\theta}(N, q)_{N}^{*}
\end{array}
$$

where $T_{\otimes}$ and $T_{\times}$denote cochain homomorphisms induced by $T_{\otimes}^{\prime}$ and $T_{\times}^{\prime}$ respectively.

4. The homomorphism $\phi_{0}^{l, m}: E_{0}^{l, m}(F) \rightarrow E_{0}^{l, m}(C)$.

Let ' $F^{m}$ be the cochain submodule of $\left(\underset{\infty}{\times} K_{\theta}(N, q, 1)\right)_{N}^{*}$ generated by the duals of simplices of $\mu_{B} \geqq m$. Put ' $F^{n-m, m}={ }_{r}\left(\underset{\infty}{\times} K_{\theta}(N, q, 1)\right)_{N}^{*} \cap^{\prime} F^{m}$ and $E_{0}^{l, m}\left({ }^{\prime} F\right)=$ ${ }^{\prime} F^{l, m} /{ }^{\prime} F^{l-1, m+1}$.

Then, from the definition of $\psi^{\prime \prime}$, follows

LEMMA 1.32 .

$$
\psi^{\prime \prime}\left({ }^{l, m}\right) \subset{ }^{\prime} C^{l, m} .
$$

More precisely, for a simplex $\rho$ of $\mu_{F}(\rho)=l$ and $\mu_{B}(\rho)=m$, we have

$$
\psi^{\prime \prime}\left(\rho^{*}\right) \equiv \xi \otimes u^{(l)} \otimes v^{(m)} \quad \bmod ^{\prime} C^{l-1, m+1} .
$$

In particular, in case $\operatorname{dim} \rho=l q+m(q+1), \xi$ can be taken as a $\Pi$-base of $W(\Pi)$ in dimension 0 .

As a direct consequence of Lemma 1.32, we have

Lemma 1.33. Let

$$
' i:\left(\left(\underset{l}{\times} M^{\prime}\right) \times\left(\underset{m}{\times} M^{\prime \prime}\right)\right)_{N}^{*} \longrightarrow F^{l, m} /^{\prime} F^{l-1, m+1}
$$

be the cochain homomorphism induced by the natural projection and let 


$$
' k: W\left(\Pi^{\prime}\right) \otimes\left(\bigotimes_{l} M_{N}^{\prime *}\right) \otimes W\left(\Pi^{\prime \prime}\right) \otimes\left(\underset{m}{\otimes} M_{N}^{\prime \prime}\right) \longrightarrow{ }^{\prime} C^{l, m} /{ }^{\prime} C^{l-1, m+1}
$$

be a $\Pi^{\prime} \times \Pi^{\prime \prime}$-equivariant cochain homomorphism induced by the natural injection. $\Pi^{\prime} \times \Pi^{\prime \prime} \subset \Pi$. Then the following diagram is homotopy commutative

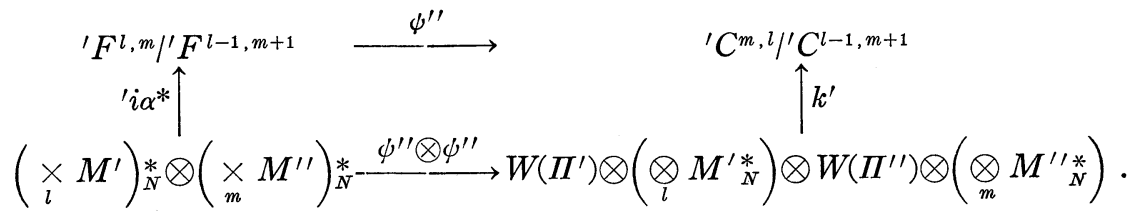

Proof. Let $\rho^{\prime}$ be a simplex of $\left(\underset{l}{\times} M^{\prime}\right)_{N}$ in dimension $l q$ and $\rho^{\prime \prime}$ be a simplex of $\left(\underset{m}{\times} M^{\prime \prime}\right)_{N}$ in dimension $m(q+1)$. Then each of the images $\phi^{\prime \prime \prime} i \alpha^{*}\left(\rho^{\prime *} \otimes \rho^{\prime \prime *}\right)$ and ${ }^{\prime} k\left(\phi^{\prime \prime} \otimes \psi^{\prime \prime}\right)\left(\rho^{\prime *} \otimes \rho^{\prime \prime *}\right)$ has the form $\xi \otimes u^{(l)} \otimes v^{(m)}$ where $\xi$ is $\Pi^{\prime} \times \Pi^{\prime \prime}$-base of $W(\Pi)$ in dimension 0 . Clearly the cochain homomorphisms $\psi^{\prime \prime}, i \alpha^{*}$ and ' $k\left(\psi^{\prime \prime} \otimes \psi^{\prime \prime}\right)$ are $\Pi^{\prime} \times \Pi^{\prime \prime}$-equivariant mod the isotropy group. Hence the well-known argument implies the required result.

Turning to the factor module, we have

Proposition 1.34. The following diagram is homotopy commutative

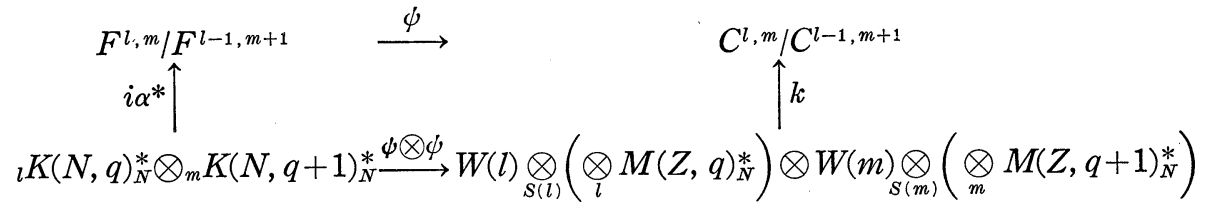

where $i$ and $k$ denote the cochain homomorphism induced by ' $i$ and ' $k$ respectively.

Let us denote by $\phi_{0}^{l, m}: E_{0}^{l, m}(F) \rightarrow E_{0}^{l, m}(C)$ the homomorphism induced from $\phi$. Then Proposition 1.34 can be restated as follows:

$$
\phi_{0}^{l, m}\left(Z_{\theta}, q\right) i \alpha^{*} \simeq k\left({ }_{l} \psi(Z, q) \otimes_{m} \psi(Z, q+1)\right)
$$

or equivalently

$$
\left.\psi_{0}^{l, m}\left(Z_{\theta}, q\right) \simeq k{ }_{l} \psi(Z, q) \otimes_{m} \psi(Z, q+1)\right) \beta^{*} i^{-1} .
$$

§. The construction of the homomorphism.

$$
{ }_{n} \varphi\left(Z_{\theta}, q\right): W(\Pi) \otimes_{I}\left(\otimes_{n} M\left(Z_{\theta}, q\right)_{N}^{*}\right) \longrightarrow\left(\underset{n}{\times} M\left(Z_{\theta}, q\right) / \Pi\right)_{N}^{*} .
$$

Let $\mathscr{K}$ be a category consisting of free $F D$-modules and $F D$-homomorphisms (transforming a simplex into a simplex or 0 ). We denote by $\mathscr{K}^{n}$ or $\times \mathscr{K}_{n}$ the category of the cartesian products $K_{1} \times \cdots \times K_{n}$ of the $F D$-modules ${ }^{n} K_{\nu}$ in $\mathscr{K}$ and the cartesian products $f_{1} \times \cdots \times f_{n}$ of the $F D$-homomorphisms $f_{\nu}$ in $\mathscr{L}$. We consider a category $\mathscr{A}$ formed as follows. An object $A$ of $\mathscr{A}$ is a pair $(K, L)$ of an $F D$-module $K$ in $\mathscr{K}^{n}$ and the sum $L$ of a finite number of the $F D$-submodules $L_{\nu}$ of $K$ in $\mathscr{K}^{n}$. Let $A=(K, L)$ and $A^{\prime}=\left(K^{\prime}, L^{\prime}\right)$ be two objects in $\mathscr{A}$. Then a map $f: A \rightarrow A^{\prime}$ is a module homomorphism $f:(K, L) \rightarrow\left(K^{\prime}, L^{\prime}\right)$ written in the form $f=f_{1} \times \cdots \times f_{n}$ in which each $f_{\nu}$ for $1 \leqq \nu \leqq n$ is a module homomorphism $f_{\nu}: K_{\nu} \rightarrow K_{\nu}^{\prime}$ which transforms a simplex into a simplex or 0 and 
comutes with $s_{i}$ but does not necessarily comute with $\partial_{i}$; Besides we require $\partial_{i} f \equiv f \partial_{i} \bmod L^{\prime}$, and that $f^{-1}\left(L^{\prime}\right)$ is a sum $F$ of a finite number of the $F D$-submodules $F_{\nu}$ of $K$ in $\mathscr{K}^{n}$. Now we define a functor $\times C$ from $\mathscr{K}^{n}$ with values in a category of differential graded modules by $(\times C)\left(K_{1} \times \cdots K_{n}\right)=K_{1} \times \cdots \times K_{n}$ and $(\times C)\left(f_{1} \times \cdots \times f_{n}\right)=f_{1} \times \cdots \times f_{n}$. In the same way, we define a functor $\otimes C$ by $(\otimes C)\left(K_{1} \times \cdots \times K_{n}\right)=K_{1} \otimes \cdots \otimes K_{n}$ and $(\otimes C)\left(f_{1} \times \cdots \times f_{n}\right)=f_{1} \otimes \cdots \otimes f_{n}$. Each of these two functors can be naturally extended to a functor defined on $\mathscr{A}$ which will be denoted by the same notation. Using the argument of acyclic models, we can prove the acyclicity of the module $\operatorname{Hom}(\times C, \otimes C)$ as in Dold [6] where $\operatorname{Hom}(\times C, \otimes C)$ is a d.g.a. module whose chain modules are

$$
\operatorname{Hom}(\times C, \otimes C)_{q}=\Pi_{r} \operatorname{Hom}\left((\times C)_{r},(\otimes C)_{r+q}\right)
$$

and the boundary operator $\partial: \operatorname{Hom}(\times C, \otimes C)_{q} \rightarrow \operatorname{Hom}(\times C, \otimes C)_{q-1}$ is defined by

$$
\partial(f)=\partial \circ f+(-1)^{q+1} f \circ \partial
$$

and the augmentation $\varepsilon: \operatorname{Hom}(\times C, \otimes C)_{q} \rightarrow Z$ is defined by

$$
\varepsilon(f)=f_{0} \in \operatorname{Hom}\left((\times C)_{0},(\otimes C)_{0}\right) \cong Z \text {. }
$$

Let $I I$ be a subgroup of $S(n)$. Then we have a natural transformation

$$
\Phi: W(\boldsymbol{I}) \otimes(\times C) \longrightarrow \otimes C
$$

which is $\Pi$-equivariant in the sense that the diagram

$$
\begin{aligned}
& W(I) \otimes(\times C)(A) \stackrel{\Phi(A)}{\longrightarrow}(\otimes C)(A) \\
& \quad x \otimes x \mid \\
& W(\Pi) \otimes(\times C)(x A) \stackrel{\Phi(x A)}{\longrightarrow}(\otimes C)(x A)
\end{aligned}
$$

is commutative for an arbitrary permutation $x \in \Pi$. Given two transformations $\Phi, \Phi^{\prime}$ as mentioned above, we have a $I$-equivariant natural homotopy $h$ : $W(I) \otimes(\times C) \rightarrow \otimes C$ between $\Phi$ and $\Phi^{\prime}$.

Now according to Dold [6] we define an outer Steenrod operation

$$
\varphi: W(\Pi) \otimes(\otimes C)^{*} \longrightarrow(\times C)^{*}
$$

by $\varphi(\xi \otimes w)(\rho)= \pm w \cdot T \Phi(\xi \otimes \rho)$. The following Lemma is proved by a familiar argument. The notations are the same as in the previous section.

Lemma 1.35. Let

$$
' i:\left(\underset{l}{\times} M^{\prime}\right) \times\left(\underset{m}{\times} M^{\prime \prime}\right) \longrightarrow F_{l, m} /^{\prime} F_{l+1, m-1}
$$

be a map of $\mathscr{A}$ induced by a natural injection and let

$$
k: W\left(\Pi^{\prime}\right) \otimes W\left(\Pi^{\prime \prime}\right) \longrightarrow W(\Pi)
$$

be a $\Pi^{\prime} \times \Pi^{\prime \prime}$-equivariant chain homomorphism induced by a natural injection $\Pi^{\prime} \times \Pi^{\prime \prime} \subset \Pi$. Then the followiug diagram is homotopy commutative

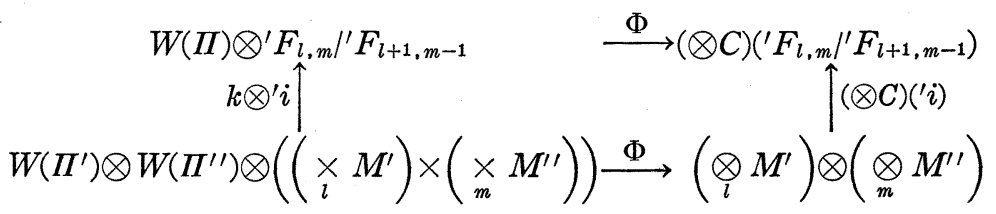


where $\omega$ denotes a natural cochain homomorphism changing factors.

From this Lemma follows:

Proposition 1.36. The following diagram is homotopy commutative

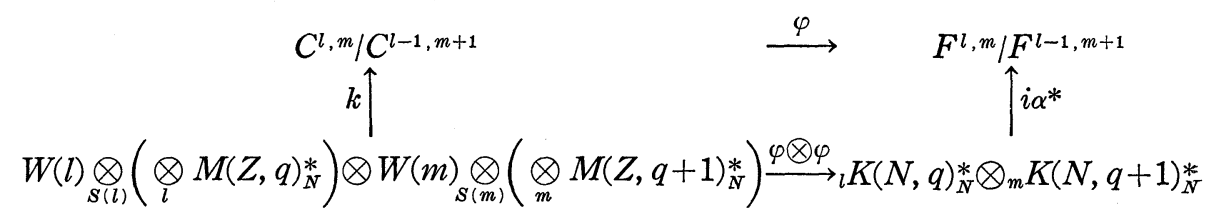

where $i$ and $k$ denote cochain homomorphisms induced by ' $i$ and ' $k$ respectively.

In other words, we have

$$
\varphi_{0}^{l, m}\left(Z_{\theta}, q\right) k \simeq i \alpha^{*}\left({ }_{l} \varphi(Z, q) \otimes_{m} \varphi(Z, q+1)\right)
$$

where $\varphi_{0}^{l, m}: E_{0}^{l, m}(C) \rightarrow E_{0}^{l, m}(F)$ denotes a homomorphism induced by $\varphi$.

\section{§ 7. Equivalence of two definitions of cohomology operations.}

Theorem 1.37. Let

$$
{ }_{n} \psi(Z, q):{ }_{n} K(N, q)_{N}^{*} \longrightarrow W(n) \underset{S(n)}{\otimes}\left(\otimes_{n} M(Z, q)_{N}^{*}\right)
$$

and

$$
{ }_{n} \varphi(Z, q): W(n) \otimes_{S(n)}\left(\otimes_{n} M(Z, q)_{N}^{*}\right) \longrightarrow{ }_{n} K(N, q)_{N}^{*}
$$

be the cochain homomorphisms defined in the preceding sections. Then the composition

$$
{ }_{n} \varphi(Z, q) \cdot{ }_{n} \psi(Z, q):{ }_{n} K(N, q)_{N}^{*} \longrightarrow{ }_{n} K(N, q)_{N}^{*}
$$

gives a cochain equivalence

Proof. To begin with, the theorem trivially holds in case $q=0$ or $n=1$. Hence we prove the theorem by double induction on $q$ and $n$.

Assume the theorem is valid for ${ }_{n}^{\prime} K\left(N, q^{\prime}\right)$ with $n^{\prime}$ and $q^{\prime}$ such that $n^{\prime} \leqq n, q^{\prime} \leqq q+1$ and $n^{\prime}+q^{\prime}<n+q+1$. Let us consider the homomorphisms

$$
\begin{aligned}
& { }_{n} \psi(0, q):{ }_{n} W(N, q)_{N}^{*} \longrightarrow W(n) \underset{S(n)}{\otimes}\left(\bigotimes_{n} M(0, q)_{N}^{*}\right) \\
& { }_{n} \varphi(0, q): W(n) \underset{S(n)}{\otimes_{n}}\left(\otimes_{n} M(0, q)_{N}^{*}\right) \longrightarrow{ }_{n} W(N, q)_{N}^{*} .
\end{aligned}
$$

Let $l$ and $m$ be the pair of nonnegative integers such that $l+m=n>1$. Then we obtain the induced homomorphisms

$$
\begin{aligned}
& \phi_{0}^{l, m}: E_{0}^{l, m}(F) \longrightarrow E_{0}^{l, m}(C) \\
& \varphi_{0}^{l, m}: E_{0}^{l, m}(C) \longrightarrow E_{0}^{l, m}(F)
\end{aligned}
$$

We proved in Propositions 1.34 and 1.36,

$$
\begin{aligned}
& \phi_{0}^{l, m}=k\left({ }_{l} \psi(Z, q) \otimes_{m} \psi(Z, q+1)\right) \beta^{*} i^{-1} \\
& \left.\varphi_{0}^{l, m}=i \alpha^{*}{ }_{l} \varphi(Z, q) \otimes_{m} \varphi(Z, q+1)\right) k^{-1}
\end{aligned}
$$


where $k^{-1}$ means a homotopy inverse of $k$. Therefore we have

$$
\varphi_{0}^{l, m} \psi^{l, m}=i \alpha^{*}\left({ }_{l} \varphi(Z, q)_{l} \psi(Z, q) \otimes_{m} \varphi(Z, q+1)_{m} \psi(Z, q+1)\right) \beta^{*} i^{-1} .
$$

Now, applying the hypothesis of induction, we can conclude that

$$
\varphi_{0}^{l, m} \psi_{0}^{l, m}: E_{0}^{l, m}(F) \longrightarrow E_{0}^{l, m}(F)
$$

gives an equivalence for $0 \leqq m<n$. Since ${ }_{n} W(N, q)$ has trivial homology, the well-known equivalence theorem between filtered differential modules proves our statement.

In the same way, we have

THEOREM 1.38. Let

$$
{ }_{n} \psi\left(Z_{\theta}, q\right):{ }_{n} K_{\theta}(N, q)_{N}^{*} \longrightarrow W(n) \underset{S(n)}{\otimes}\left(\bigotimes_{n} M\left(Z_{\theta}, q\right)_{N}^{*}\right)
$$

and

$$
{ }_{n} \varphi\left(Z_{\theta}, q\right): W(n) \underset{S(n)}{\otimes}\left(\bigotimes_{n} M\left(Z_{\theta}, q\right)_{N}^{*}\right) \longrightarrow{ }_{n} K_{\theta}(Z, q)_{N}^{*}
$$

be the cochain homomorphisms defined as before. Then the composition

$$
{ }_{n} \varphi\left(Z_{\theta}, q\right) \cdot{ }_{n} \psi\left(Z_{\theta}, q\right):{ }_{n} K_{\theta}(N, q) *{ }_{N}^{*} \longrightarrow K_{\theta}(N, q)_{N}^{*}
$$

gives a cochain equivalence.

Proof. We consider the induced homomorphisms

$$
\begin{aligned}
& \psi_{0}^{l, m}: E_{0}^{l, m}(F) \longrightarrow E_{0}^{l, m}(C) \\
& \varphi_{0}^{l, m}: E_{0}^{l, m}(C) \longrightarrow E_{0}^{l, m}(F)
\end{aligned}
$$

defined by ${ }_{n} \psi\left(Z_{\theta}, q\right)$ and ${ }_{n} \varphi\left(Z_{\theta}, q\right)$ respectively. Then by Propositions $1.34,1.36$ and Theorem 1.37, we conclude that

$$
\varphi_{0}^{l, m} \psi_{0}^{l, m}: E_{0}^{l, m}(F) \longrightarrow E_{0}^{l, m}(F)
$$

gives an equivalence for each pair of nonnegative integers $l$ and $m$ such that $l+m=n$. This leads to our assertion.

Finally we prove

THEOREм 1.39. Let $K$ be a free FD-module whose homology groups are of finite type. Then there exists a cochain homomorphism

$$
\phi:(\underset{n}{\times} K / S(n)) * \longrightarrow W(n) \underset{S(n)}{\otimes}\left(\underset{n}{\otimes} K^{*}\right)
$$

which has the following property.

Let

$$
\varphi: W(n) \underset{S(n)}{\otimes}\left(\underset{n}{\otimes} K^{*}\right) \longrightarrow(\underset{n}{\times} K / S(n)) *
$$

be the outer Steenrod operation. Then the compasition

$$
\varphi \phi:(\underset{n}{\times} K / S(n)) * \longrightarrow(\underset{n}{\times} K / S(n)) *
$$

gives a cachain equivalence. 
Proof. Suppose given a direct sum decomposition $K=K^{\prime}+K^{\prime \prime}$. Then we know the following equivalences.

$$
\begin{aligned}
\left(\mathrm{SP}^{n} K\right)^{*} & \simeq \sum_{l+m}\left(\mathrm{SP}^{l} K^{\prime}\right)^{*} \otimes\left(\mathrm{SP}^{m} K^{\prime \prime}\right)^{*} \\
W(n) \bigotimes_{S(n)}\left(\bigotimes_{n} K^{*}\right) & \simeq \sum W(l) \bigotimes_{S(l)}\left(\bigotimes_{l} K^{*}\right) \otimes W(m) \otimes_{S(m)}\left(\bigotimes_{m} K^{\prime \prime}\right) .
\end{aligned}
$$

On the other hand, from the assumption, $K$ is $F D$-homotopy equivalent to the direct sum of elementary $F D$-modules $M\left(Z_{\theta}, q\right)$.

According to Dold [5], $K \simeq K^{\prime}$ implies $\underset{n}{\times} K / \Pi \simeq \times \underset{n}{\times} K^{\prime} / \Pi$ for $\Pi \subset S(n)$.

Now, applying Theorem 1.38 to these results, we can prove the existence of the homomorphism $\phi$ with the required property.

\section{Chapter 2. A cell-decomposition of $\underset{n}{\times} S^{q}$}

In this chapter, we shall study a cell decomposition $S(q, n)$ of $\times S^{q}$ as a $C W$-complex. Any element of the transformation group $S(n)$ operating on $\times S^{q}$ will transform any cell of $S(q, n)$ into a cell of $S(q, n)$. We shall use this " cell decomposition $S(q, n)$ to define a free chain module $W^{+}(n, q)$ over $Z(S(n))$. The direct limit $\lim _{q \rightarrow \infty} W^{+}(n, q)$ will give a projective resolution of $Z$ over $Z(S(n))$, which is "simplest" in a certain sense. We shall define a d.g.a. algebra $A^{\prime}(q)$ to be the $(q-1)$-fold bar construction $\bar{B}^{(q-1)}(E([\alpha]))$ of the exterior algebra $E([\alpha])$ of generator $[\alpha]$ in dimension $1 . A^{\prime}(N, q)$ is a d.g.a. algebra with multiplicity and the natural injection $A^{\prime}(N, q) \rightarrow A(N, q)$ gives a chain homotopy equivalence preserving multiplicity. Our objective is to prove an important relation

$$
{ }_{n} A^{\prime}(N, q) \cong W^{+}(n, q) \underset{S(n)}{\otimes}\left(\bigotimes_{n} M(Z, q)_{N}^{*}\right) .
$$

Roughly speaking, the cohomology of the left hand side of (1) defines the cohomology operation in Eilenberg's sense and that of the right hand side those in Steenrod's, sense, so that (1) clarifies the relationship between the two. In this chapter, we shall also develop some consequences of (1) which will be used in the next chapter to define the cohomology operations of higher orders.

\section{$\S 1$. A cell decomposition of $\underset{n}{\times} \boldsymbol{R}^{q}$}

1. Cell-complex $R(q, n)$

Since $S^{q}$ is a one-point-compactification of $R^{q}$, we shall begin with the construction of a cell decomposition of $\times R^{q}$ which will be denoted by $R(q, n)$. $R(q, n)$ is defined to be a collection of a finite number of cells each of which is the intersection of a finite number of affine subspaces and halfspaces of $\times R^{q}$.

First we introduce a lexicographic order in $R^{q}$ as usual. Let $\boldsymbol{a}=\left(a^{1}, \cdots, a^{q}\right)$ and $\boldsymbol{b}=\left(b^{1}, \cdots, b^{q}\right)$ be two points of $R^{q}$. By definition $\boldsymbol{a}<\boldsymbol{b}$ if and only if there exists a nonnegative integer $\rho$ such that $a^{\sigma}=b^{\sigma}$ for $\sigma \leqq \rho$ and $a^{\rho+1}<b^{\rho+1}$. Then an arbitrary point of $\times R^{q}$ can be written in the form

$$
x\left(\boldsymbol{a}_{1}, \cdots, \boldsymbol{a}_{n}\right)=\left(\boldsymbol{a}_{x^{-1}(1)}, \cdots, \boldsymbol{a}_{x^{-1}(n)}\right)
$$


where $x \in S(n)$ and $\boldsymbol{a}_{\nu}=R^{q}$ for $1 \leqq \nu \leqq n$ satisfying the relation

$$
\boldsymbol{a}_{\nu} \geqq \boldsymbol{a}_{\nu+1}
$$

We set $\boldsymbol{a}_{\nu}=\left(a_{\nu}^{1}, a_{\nu}^{2}, \cdots, a_{\nu}^{q}\right)$. Then we can define an ordered set of integers $\left(r_{1}, r_{2}, \cdots, r_{n}\right)$ with $0 \leqq r_{\nu} \leqq q$ for $1 \leqq \nu \leqq n$ and $r_{1}=q$ which is determined by the following relations

$$
\left\{\begin{array}{lr}
a_{\nu-1}^{\sigma}=a_{\nu}^{\sigma} & \text { for } 1 \leqq \sigma \leqq \rho_{\nu} \\
a_{\nu-1}^{\rho_{\nu}+1}>a_{\nu}^{\rho_{\nu}+1} & \text { if } \rho_{\nu}<q
\end{array}\right.
$$

where $\rho_{\nu}=q-r_{\nu}$.

Conversely, given an ordered set of integers $\left(r_{1}, \cdots, r_{n}\right)$ such that $0 \leqq r_{\nu} \leqq q$ with $1 \leqq \nu \leqq n$ and $r_{1}=q$, we can define a cell $\alpha=\left(r_{1}, \cdots, r_{n}\right)$ to be the set of all the points $P=\left(\boldsymbol{a}_{1}, \cdots, \boldsymbol{a}_{n}\right) \in \times R^{q}$ satisfying the relations (C). Given $x \in S(n)$, the cell $x \alpha$ will stand for the set of the points expressed as $x P$ in terms of $x \in S(n)$ and $P \in \alpha$. The collection of all the cells of the form $x \alpha$ will be denoted by $R(q, n)$.

Clearly $R(q, n)$ satisfies the following conditions:

$x \alpha$ is homeomorphic to an open disk of dimension $\sum_{\nu=1}^{n} \gamma_{\nu}$ which will be called the dimension of $x \alpha$ and will be denoted by $|x \alpha|$.

An arbitrary point of $\times R^{q}$ is contained in some $x \alpha$.

Two distinct cells $x \alpha$ and $y \beta$ are disjoint.

A cell $x \alpha$ is the intersection of a finite number of the subspaces and halfspaces of $\times R^{q}$.

The boundary of a cell $x \alpha$ is contained in the union of cells $y \beta$ of dimensions lower than $x \alpha$.

Thus we have obtained a locally finite cell decomposition $R(q, n)$ of $\times R^{q}$.

2. Incidence relations

To begin with, we introduce some notations.

Suppose given a cell $\alpha \in R(q, n)$ of the form $\left(r_{1}, \cdots, r_{n}\right)$.

Let $r$ be an integer such that $0 \leqq r \leqq q$. Then we define a monotone increasing sequence

$$
\nu(r, 1), \cdots, \nu(r, i), \cdots, \nu(r, \imath(r))
$$

consisting of all the indices $\nu$ 's such that $r_{\nu} \geqq r$. Now we put

$$
n(r, i)=\nu(r, i+1)-\nu(r, i)
$$

where $\nu(r, \imath(r)+1)$ means $n+1$.

Let $\pi$ be a permutation of the form

$$
\pi=\left(\begin{array}{c}
1, \cdots, \ell(r) \\
\pi(1), \cdots, \pi(\ell(r))
\end{array}\right) .
$$

Then we define a permutation $x_{\alpha}(r, \pi) \in S(n)$ by the formula

$$
x_{\alpha}(r, \pi)\left(\sum_{j=1}^{i-1} n(r, j)+\lambda\right)=\sum_{j=1}^{\pi(i)-1} n\left(r, \pi^{-1}(j)\right)+\lambda
$$

for $1 \leqq \lambda \leqq n(r, i)$ and its signature will be denoted by $\varepsilon_{\alpha}(r, \pi)$. 
Under these conventions, we shall determine the form of the cells $y \beta$ which are contained in the boundary $\partial \alpha$ of $\alpha$. A cell $y \beta$, which is contained in $\partial \alpha$ and has $\operatorname{dim} \beta=\operatorname{dim} \alpha-1$, will be called a face of $\alpha$. We consider a point $Q$ belonging to a face $y \beta$ of $\alpha$ which is written in the form $\left(c_{1}, \cdots, c_{n}\right)$ with $c_{\nu} \in R^{q}$. We write $\boldsymbol{c}_{\mu}=\left(c_{\mu}^{1}, \cdots, c_{\mu}^{q}\right)$ then there exists a certain index $\nu$ with $\rho_{\nu}<q$ for which

$$
\begin{aligned}
c_{\nu-1} & =c_{\nu} \\
c_{\nu-1}^{\rho_{\nu}+2} & \neq c_{\nu}^{\rho_{\nu}+2}
\end{aligned} \quad \text { for } 1 \leqq \sigma \leqq \rho_{\nu}+1
$$

holds, and for any $\mu \neq \nu$, we have

$$
\begin{array}{cr}
c_{\mu-1}^{\sigma}=c_{\mu}^{\sigma} & \text { for } 1 \leqq \sigma \leqq \rho_{\mu} \\
c_{\mu-1}^{\rho_{\mu+1}}>c_{\mu}^{\rho_{\mu}+1} & \text { if } \rho_{\mu}<q .
\end{array}
$$

From now on, we write $\rho_{\nu}$ as $\rho$ and $r_{\nu}=q-\rho_{\nu}$ as $r$ for the sake of simlicity. Then we have

$$
\nu=\nu(r, i)
$$

for some index $i$. Furthermore, we have

$$
\begin{aligned}
& \nu(r, i-1)=\nu(r-1, u) \\
& \nu(r, i)=\nu(r-1, v) \\
& \nu(r, i+1)=\nu(r-1, w)
\end{aligned}
$$

for some indices $u, v, w$.

If we use these notations, the components of $Q=\left(c_{1}, \cdots, c_{n}\right)$ satisfy the following relations. From the definition follows that, if $\nu(r, j-1)<\mu<\nu(r, j)$ for some $j$, we have

$$
c_{\mu-1}^{\sigma}=c_{\mu}^{\sigma} \quad \text { for } \sigma \leqq \rho+1
$$

and, if $\mu=\nu(r, j)$ for some $j$, we have

$$
\begin{array}{lr}
c_{\mu-1}^{\sigma}=c_{\mu}^{\sigma} & \text { for } \sigma \leqq \rho_{\mu} \\
c_{\mu-1}^{\rho_{\mu}+1}>c_{\mu}^{\rho \mu+1} & \text { since } \rho_{\mu} \leqq \rho<q,
\end{array}
$$

with the sole exception $\mu=\nu=\nu(r, i)$ for which we have

$$
c_{\nu-1}^{\rho+1}=c_{\nu}^{\rho+1}
$$

since $\rho_{\nu}=\rho$.

Thus we obtain

$$
\boldsymbol{c}_{\lambda}>\boldsymbol{c}_{\mu}
$$

if $\lambda<\nu(r, i-1) \leqq \mu$ or $\lambda<\nu(r, i+1) \leqq \mu$. Moreover the definition implies that if $\nu(r-1, k-1)<\mu<\nu(r-1, k)$ for some $k$

$$
c_{\mu-1}^{\sigma}=c_{\mu}^{\sigma} \quad \text { for } \sigma \leqq \rho+2
$$

and if $\mu=\nu(r-1, k)$ for some $k$ such that $u<k<v$ or $v<k<w$

$$
\begin{array}{lr}
c_{\mu-1}^{\sigma}=c_{\mu}^{\sigma} & \text { for } \sigma \leqq \rho+1 \\
c_{\mu-1}^{\rho+2}<c_{\mu}^{\rho+2} & \text { if } \rho_{\mu}=\rho+1>\rho .
\end{array}
$$

Hence we can define a permutation 


$$
\pi=\left(\begin{array}{ccccc}
u & \cdots & v & \cdots & w-1 \\
\pi(u) & \cdots & \pi(v) & \cdots & \pi(w-1)
\end{array}\right)
$$

so as to satisfy

$$
c_{y(\mu-1)}^{\rho+2}>c_{y(\mu)}^{\rho+2}
$$

if we set $y^{-1}=x_{a}(r-1, \pi)$. Here it should be noted that we have

$$
\begin{aligned}
& \pi(u)<\cdots<\pi(v-1) \\
& \pi(v)<\cdots<\pi(w-1) .
\end{aligned}
$$

Since the components of

$$
y^{-1} Q=\left(c_{y(1)}, \cdots, c_{y(n)}\right)
$$

satisfy the relation $\boldsymbol{c}_{y(\mu-1)} \geqq \boldsymbol{c}_{y(\mu)}$ for $1 \leqq \mu \leqq n$, we find the cell

$$
\beta=\left(s_{1}, \cdots, s_{n}\right)
$$

to which $y^{-1} Q$ belongs. Here the set of integers $s_{\mu}$ is defined as follows:

$$
s_{\mu}= \begin{cases}r_{y(\mu)} & \text { if } y(\mu) \neq \nu \\ r_{\nu}-1 & \text { if } y(\mu)=\nu\end{cases}
$$

\section{Incidence numbers}

Let $\alpha$ be a cell of the form $\left(r_{1}, \cdots, r_{n}\right)$. We define an orientation of a cell $x \alpha$ by the coordinate system given as follows:

$$
\begin{gathered}
a_{1}^{1}, \quad \cdots, a_{1}^{q}, \\
\cdots \\
a_{\mu}^{\rho_{\mu}+1}, \cdots, a_{\mu}^{q}, \\
\cdots \\
a_{n}^{\rho_{n}+1}, \cdots, a_{n}^{q} .
\end{gathered}
$$

Let $y \beta$ be a face of $\alpha$ mentioned in the previous paragraph. In order to find the incidence number $[\alpha: y \beta]$, we begin with the re-orientation $\varepsilon \alpha$ of $\alpha$ obtained by interchanging the order of coordinate functions as follows:

$$
\begin{gathered}
a_{1}^{1}, \quad \cdots, a_{1}^{q}, \\
\cdots \\
a_{\mu}^{\rho_{\mu}+1}, \cdots, \widehat{a_{\mu}^{\rho}+1}, \cdots, a_{\mu}^{q}, \\
\cdots \\
\cdot a_{\mu}^{\rho+1}, a_{\nu}^{\rho+1}, \cdots, a_{\nu}^{q}, \\
\cdots \\
a_{n}^{\rho_{\mu}+1}, \cdots, a_{n}^{q}
\end{gathered}
$$

where $\mu=\nu(r, i-1)$ and $\nu=\nu(r, i)$. Clearly $\varepsilon \alpha$ induces a reorientation $\delta y \beta$ of $y \beta$ by deleting the coordinate function $a_{\nu}^{\rho+1}$. Now the incidence number $[\varepsilon \alpha: \delta y \beta]$ is given by

$$
[\varepsilon \alpha: \delta y \beta]=(-1)^{\lambda \sum^{\nu=1} r_{\lambda}}
$$


Direct computation shows that

$$
\delta=\varepsilon \cdot \varepsilon_{\infty}(r-1, \pi)
$$

Now we put

$$
\begin{gathered}
s_{\alpha, j}=r+\sum_{\nu(r, j)<\lambda<(r, j+1)} r_{\lambda} \\
\varepsilon_{\alpha}(r-1, \pi)=(-1)^{\Sigma^{\prime} s_{r-1, j} \cdot s_{r-1, k} .}
\end{gathered}
$$

where the summation is extended over the set of couples $(j, k)$ such that $u \leqq j<v$, $v \leqq k<w$ and $\pi(j)>\pi(k)$.

Summarizing the results, we obtain

$$
[\alpha: y \beta]=(-1)^{\lambda=1} \sum^{\nu-1} r_{1}+\Sigma^{\prime} s_{r-1}, j^{s} \cdot r-1, k
$$

4. A decomposition formula for $R(q, n)$

In order to obtain more results concerning the structure of $R(q, n)$, we introduce the following decomposition formula for $R(q, n)$ which shows us the complete parallelism between $R(q, n)$ and ${ }_{n} A(N, q)$.

First we consider the case $q=0$. In this case, only one cell of $R(0, n)$ is of the form $(0, \cdots, 0)$ which can be identified with the natural number $n$. On the other hand we know ${ }_{n} A(N, 0)=Z\left((\alpha-e)^{n}\right)$. Thus we have an isomorphism

$$
R(0, n) \cong{ }_{n} A(N, 0) \text {. }
$$

Next we assume $q>0$. Let $\alpha \in R(q, n)$ be a cell of the form $\left(r, \cdots, r_{n}\right)$. Then a sequence

$$
\nu(q, 1), \cdots, \nu(q, \iota(q))
$$

is defined to be the set of $\nu^{\prime} s$ such that $r_{\nu} \geqq q$ i.e. $r_{\nu}=q$. Now we put $n_{i}=$ $\nu(q, i+1)-\nu(q, i)$. Then $\alpha_{i} \in R\left(q-1, n_{i}\right)$ is defined by

$$
\alpha_{i}=\left(q-1, r_{\nu(q, i)+1}, \cdots, r_{\nu(q, i+1)-1}\right) \text {. }
$$

Obviously, a sequence of $\alpha_{i}$ determines $\alpha$, so we write

$$
\alpha=\left[\alpha_{1}|\cdots| \alpha_{\iota(q)}\right] \text {. }
$$

Here we note that the dimension of $\alpha$ is given by

$$
\operatorname{dim} \alpha=\sum_{i=1}^{\iota(q)} \operatorname{dim} \alpha_{i}+\iota(q) \text {. }
$$

As easily seen this correspondence yields an isomorphism

$$
R(q, n) \cong \sum_{n_{1}+\cdots+n_{\iota}=n} Z(S(n)){ }_{S\left(n_{1}\right) \times \cdots \times S\left(n_{\iota}\right)}\left(R\left(q-1, n_{1}\right) \otimes \cdots \otimes R\left(q-1, n_{\iota}\right)\right)
$$

where $\iota$ denotes $\iota(q)$. This is the decomposition formula for $R(q, n)$.

Let $\alpha \in R(q, l)$ and $\beta \in R(q, m)$ be two cells of the form $\alpha=\left(r_{1}, \cdots, r_{l}\right)$ and $\beta=\left(s_{1}, \cdots, s_{m}\right)$ respectively. We define the product $\alpha \beta \in R(q, l+m)$ of $\alpha$ and $\beta$ as follows. We can define monotone increasing sequences

$$
\begin{aligned}
& \lambda(q, 1), \cdots, \lambda(q, \iota) \\
& \mu(q, 1), \cdots, \mu(p, \kappa)
\end{aligned}
$$

consisting of all the $\lambda^{\prime} s$ and $\mu^{\prime} s$ such that $r_{\lambda}=q$ and $s_{\mu}=q$ respectively. We 
put

$$
\begin{gathered}
l_{i}=\lambda(q, i+1)-\lambda(q, i) \\
m_{k}=\mu(q, k+1)-\mu(q, k)
\end{gathered}
$$

and

$$
n_{j}=\left\{\begin{array}{l}
l_{j} \\
m_{j-\imath}
\end{array}\right.
$$

for $1 \leqq j \leqq \imath$

for $\iota+1 \leqq j \leqq \imath+\kappa$

Let $\pi \in S(\iota+\kappa)$ be a permutation

$$
\pi=\left(\begin{array}{c}
1, \cdots, \quad \iota, \quad \iota+1, \cdots, \iota+\kappa \\
\pi(1), \cdots, \pi(\iota), \pi(\iota+1), \cdots, \pi(\iota+\kappa)
\end{array}\right)
$$

satisfying the relation

$$
\begin{gathered}
\pi(1)<\cdots<\pi(\iota) \\
\pi(\iota+1)<\cdots<\pi(\iota+\kappa)
\end{gathered}
$$

$\pi$ determines a permutation $x(\pi) \in S(l+m)$ defined by

$$
x(\pi)\left(\sum_{j=1}^{i-1} n_{j}+\lambda\right)=\sum_{j=1}^{\pi(i)-1} n_{\pi^{-1}(j)}+\lambda
$$

$1 \leqq \lambda \leqq n_{i}$

whose signature is denoted by $\varepsilon(\pi)$.

$$
\begin{aligned}
& \alpha=\left[\alpha_{1}|\cdots| \alpha_{\iota}\right] \\
& \beta=\left[\beta_{1}|\cdots| \beta_{\aleph}\right]
\end{aligned}
$$$$
\beta_{k} \in R\left(q-1, m_{k}\right) \text {. }
$$

Employing the notation

$$
\gamma_{j}=\left\{\begin{array}{l}
\alpha_{j} \\
\beta_{j-1}
\end{array}\right.
$$

for $\quad 1 \leqq j \leqq \iota$

for $\iota+1 \leqq j \leqq \imath+\kappa$

we have

$$
\varepsilon(\pi)=(-1)^{\Sigma\left(\left|\gamma_{j}\right|+1\right)\left(\left|\gamma_{k}\right|+1\right)}
$$

where the summation extends over the set of couples $(j, k)$ such that $1 \leqq j \leqq \iota$, $\iota+1 \leqq k \leqq \iota+\kappa$ and $\pi(j)>\pi(k)$.

$$
\alpha \beta=\sum \varepsilon(\pi) x(\pi)\left[\gamma_{\pi(1)}|\cdots| \gamma_{\pi(\iota+k)}\right] .
$$

Easy computation shows that the product defines a chain homomorphism

$$
R(q, l) \otimes R(q, m) \longrightarrow R(q, l+m) .
$$

The decomposition formula for $R(q, n)$ and the notion of product lead us a simplified formula for the boundary orerator of $R(q, n)$, i.e.

$$
\begin{aligned}
& \partial\left[\alpha_{1}|\cdots| \alpha_{\imath}\right] \\
& \quad=\sum_{i=1}^{i}(-1)^{\left|\alpha_{1}\right|+\cdots+\left|\alpha_{i-1}\right|+i}\left[\alpha_{1}|\cdots| \partial \alpha_{i}|\cdots| \alpha_{\iota}\right] \\
& \quad+\sum_{i=1}^{i-1}(-1)^{\left|\alpha_{1}\right|+\cdots+\left|\alpha_{i}\right|+i}\left[\alpha_{1}|\cdots| \alpha_{i} \alpha_{i+1}|\cdots| \alpha_{\imath}\right]
\end{aligned}
$$

which has the same form as the boundary formula on the bar construction. 
Starting from the isomorphism

$$
R(0, n) \underset{S(n)}{\otimes} Z \cong{ }_{n} A(N, 0)
$$

we reach inductively the required result

$$
R(q, n) \underset{S(n)}{\otimes} Z \cong{ }_{n} A(N, q) \text {. }
$$

\section{§2. Cell-decompositions of $\times S^{q}$ and $\times D^{q}$}

Now we return to our first objective which is to find the decomposition of $\times S^{q}$.

Since $S^{q}$ is a one-point-compactification of $R^{q}$ obtained by attaching a point at infinity $\infty$, we introduce an order in the set of points of $S^{q}$ so as to extend the order of $R^{q}$ by setting $P>\infty$ if $P \neq \infty$.

Let $m$ be a non-negative integer such that $m \leqq n$. Suppose given an ordered set of integers $\left(r_{1}, \cdots, r_{m}\right)$ such that $0 \leqq r_{\nu} \leqq q$ for $1 \leqq \nu \leqq m$ and $r_{1}=q$. Then we define a cell $\alpha=\left(r_{1}, \cdots, r_{m}\right)$ to be the set of consisting of all the points $P=$ $\left(P_{1}, \cdots, P_{n}\right) \in \times S^{q}$ with $P_{\nu} \in S^{q}$ satisfying the relation $\left(P_{1}, \cdots, P_{m}\right) \in\left(r_{1}, \cdots, r_{m}\right)$ and $P_{\nu}=\infty$ for $m<\nu \leqq n$.

Suppose given a cell $\alpha$ and an element $x \in S(n)$. Then $x \alpha$ is the set of all the points $x P$ with $P \in \alpha$. The collection of all the cells of the form $x \alpha$ constitutes a cell-complex whose underlying space is $\times S^{q}$. This complex will be denoted by $S(q, n)$. Obviously $R(q, m)$ is considered as a subcollection of $S(q, n)$ if $m \leqq n$ and the union of $x R(q, m)$ with $x \in S(n)$ and $m \leqq n$ covers $S(q, n)$.

A cell $y \beta$ is called a face of $x a$, if $y \beta$ is contained in $\partial x \alpha$ and $\operatorname{dim} \beta=$ $\operatorname{dim} \alpha-1$. Let $x \alpha$ and $y \beta$ be two cells of $R(q, m)$ for $m \leqq n$. If $y \beta$ is a face of $x \alpha$ with respect to the incidence relation in the complex $R(q, m)$, then $y \beta$ becomes a face of $x \alpha$ also in $S(q, n)$.

Let $\alpha \in S(q, n)$ be a cell of the form $\left(r_{1}, \cdots, r_{m}\right)$ with $m \leqq n$ and $y \beta$ be a face of $\alpha$. We consider a point $Q$ of $y \beta$ which is written in the form $\left(Q_{1}, \cdots, Q_{m}, \infty, \cdots, \infty\right)$ with $Q_{\nu} \in S^{q}$ and want to find a face $y \beta$ of $\alpha$ containg $Q$.

If all the components $Q_{\nu}$ with $1 \leqq \nu \leqq m$ are points of $R^{q} \subset S^{q}$, we can find the face $y \beta$ of $\alpha$ containing $Q$ in $R(q, m)$.

So we only need to consider the case in which at least one and consequently exactly one among the components $Q_{\nu}$ with $1 \leqq \nu \leqq m$ is the point $\infty$. From the dimensional reason we have $Q_{\nu}=\infty$ for some $\nu$ of the form

$$
\nu=\nu(1, v)-1 \quad \text { or } \quad \nu(1, v)
$$

and there is an index $i$ determined by

$$
\nu(2, i)<\nu<\nu(2, i+1) .
$$

Hence we have two indices $u$ and $w$ defined by

$$
\begin{aligned}
\nu(2, i) & =\nu(1, u) \\
\nu(2, i+1) & =\nu(1, w) .
\end{aligned}
$$

Let $P$ be a point of $\alpha$. As before, we write $P=\left(\boldsymbol{a}_{1}, \cdots, \boldsymbol{a}_{m}, \infty, \cdots, \infty\right)$ and $\boldsymbol{a}_{\mu}=\left(a_{\mu}^{1}, \cdots, a_{\mu}^{q}\right)$. Then as in $\S 1$ we can show the following: If $\nu(1, k-1)<\mu<(1, k)$ for some $k$, we have 


$$
\boldsymbol{a}_{\mu-1}=\boldsymbol{a}_{\mu},
$$

and if $\mu=\nu(1, k)$ for some $k$ such that $u<k<w$, we have

$$
\begin{array}{ll}
a_{\mu-1}^{\sigma}=a_{\mu}^{\sigma} & \text { for } 1 \leqq \sigma \leqq q-1 \\
a_{\mu-1}^{q}>a_{\mu}^{q} . &
\end{array}
$$

Now we note that the assumption $Q_{\nu}=\infty$ implies $v=u+1$ or $v=w-1$, accordingly $\nu=\nu(1, v)-1$ or $\nu=(1, v)$.

We consider the first case $\nu=\nu(1, v)-1$. Then the point $Q$ is obtained by taking $\lim a_{\nu}^{q}=+\infty$ in $\alpha$. Hence we can define a permutation

$$
\pi=\left(\begin{array}{cc}
u+1, u+2, \cdots, \iota(1) & , u \\
u, u+1, \cdots, \iota(1)-1, \iota(1)
\end{array}\right)
$$

such that the point

$$
y^{-1} Q=\left(Q_{y(1)}, \cdots, Q_{y(n)}\right)
$$

with $y^{-1}=x(1, \pi)$ belongs to some cell

$$
\beta=\left(s_{1}, \cdots, s_{l}\right)
$$

which is determined by

$$
s_{\lambda}=\left\{\begin{array}{l}
r_{y(\lambda)} \\
r_{\lambda}
\end{array}\right.
$$

for $\lambda \neq \nu(1, u)$

for $\lambda=\nu(1, u)$

for $1 \leqq \lambda \leqq l$ with $l=m-n(1, u)$ where $\mathrm{n}(1, u)=\nu(1, u+1)-\nu(1, u)$. The incidence number is given by

$$
[\alpha: y \beta]=(-1)^{\lambda \sum^{\lambda-1} r_{\lambda}-1} .
$$

Next we consider the case $\nu=\nu(1, v)$. Now it suffices to take $\lim a_{\nu}^{\boldsymbol{q}}=-\infty$. We define

$$
\pi=\left(\begin{array}{c}
w, w+1, \cdots, \iota(1), w-1 \\
w-1, w, \cdots, \iota(1)-1, \iota(1)
\end{array}\right)
$$

and we set $y^{-1}=x(1, \pi)$. Then the point $y^{-1} Q$ belongs to a cell

which is determined by

$$
\beta=\left(s_{1}, \cdots, s_{l}\right)
$$

$$
s_{\lambda}=r_{y(\lambda)}
$$

for $1 \leqq \lambda \leqq l$ with $l=m-n(1, w-1)$ where $n(1, w-1)=\nu(1, w)-\nu(1, w-1)$. The incidence number is given by

$$
[\alpha: y \beta]=(-1)^{\lambda \sum^{\lambda=1} r_{\lambda}}
$$

Suppose given a point $P \in \times S^{q}$ of the form $\left(P_{1}, \cdots, P_{n}\right)$. Then $\Lambda(P)$ denotes the set of indices $\nu$ 's such that $P_{\nu}=\infty$.

Let $\Lambda$ be a subset of $\{1, \cdots, n\}$ and let $S(\Lambda)$ be the group of permutations of $\Lambda$. The subset of $\times S^{q}$ consisting of points $P$ with $\Lambda(P)=\Lambda$ constitute a subcomplex of $S(q, n)$ which will be denoted by $S(q, n, \Lambda)$. Let $M$ be the com- 
plementary set of $\Lambda$. Then maximal subgroup of $S(n)$ which leaves $S(q, n, \Lambda)$ invariant is $S(M) \times S(\Lambda)$ where $S(\Lambda)$ operates trivially on $S(q, n, \Lambda)$.

Now we put $\Lambda(m)=\{m+1, \cdots, n\}$. Then we have a module isomorphism

$$
e: R(q, m) \longrightarrow S(q, n, \Lambda(m)) \text {. }
$$

Let $x_{i}$ be a representative of a left coset of $S(n)$ with respect to $S(m) \times S(n-m)$. Then we obtain a canonical isomorphism

$$
x_{i}: S(q, n, \Lambda(m)) \longrightarrow S\left(q, n, x_{i} \Lambda(m)\right) .
$$

Then the above isomorphisms imply

$$
S(q, n) \cong \sum_{m=0}^{n} Z(S(n)){\underset{S(m) \times S(n-m)}{\otimes}}_{\otimes} R(q, m)
$$

or equivalently

$$
S(q, n) \cong \sum_{m=0}^{n} Z(S(n)){ }_{S\left(M_{m}\right) \times S\left(\Lambda_{m}\right)} S\left(q, n, \Lambda_{m}\right)
$$

where $\Lambda_{m}$ is an arbitrary subset of $\{1, \cdots, n\}$ such that $\|_{m}=n-m$ and $M_{m}$ is the complementary set of $\Lambda_{m}$.

The decomposition formula for $S(q, n)$ can be stated as follows :

For $q=0$, we have

$$
S(0, n, \Lambda(m)) \cong Z(S(n)) \underset{S(m) \times S(n-m)}{\bigotimes} Z\left(\alpha^{m}\right) .
$$

For $q>0$, we have

$$
\begin{aligned}
& S(q, n, \Lambda) \\
& \quad \cong \sum(S(M)){ }_{S\left(M_{1}\right) \times \cdots \times S\left(M_{\iota}\right)} S\left(q-1, n_{0}, \Lambda_{0}\right) \otimes\left(q-1, n_{1}, \Lambda_{1}\right) \otimes \cdots \otimes S\left(q-1, n_{\iota}, \Lambda_{\iota}\right)
\end{aligned}
$$

where the summation extends over the set of ordered sets $\left(n_{1}, \cdots, n_{\imath}\right)$ of positive integers $n_{i}$ such that $n_{1}+\cdots+n_{\iota} \leqq n$ and the set of subsets $\Lambda_{i}$ of $\left\{1, \cdots, n_{i}\right\}$ satisfying the conditions $\Lambda_{0}=\Lambda(0)$ and $\Lambda_{0} \cup \Lambda_{1} \cup \cdots \cup \Lambda_{\iota}=\Lambda$ where we identify the suffix $\lambda \in \Lambda_{i}$ with $\sum_{j=1}^{i-1} n_{j}+\lambda$.

Now we define a multiplication

$$
S(q, l) \otimes S(q, m) \longrightarrow S(q, l+m)
$$

to be a $S(l) \times S(m)$-equivariant chain homomorphism for which we have the commutative diagram

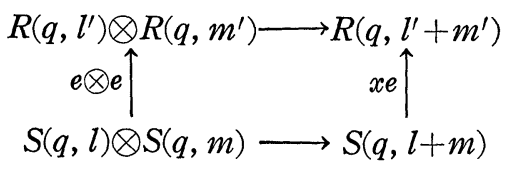

where $x$ denotes a permutation

$$
x(\lambda)=\left\{\begin{array}{lr}
\lambda & 1 \leqq \lambda \leqq l^{r} \\
l+\left(\lambda-l^{\prime}\right) & l^{\prime}+1 \leqq \lambda \leqq l^{\prime}+m^{\prime} \\
l+\left(\lambda-l^{\prime}-m^{\prime}\right) & l^{\prime}+m^{\prime}+1 \leqq \lambda \leqq l+m^{\prime} \\
\lambda & l+m^{\prime}+1 \leqq \lambda \leqq l+m
\end{array}\right.
$$


and the upper arrow in the diagram means a multiplication defined in the paragraph 4 of $\S 1$.

Next we define an augmentation

$$
\varepsilon: S(0, n) \longrightarrow Z(S(n))
$$

to be a $S(n)$-equivariant chain homomorphism for which we have the commutative diagram

for $m \leqq n$.

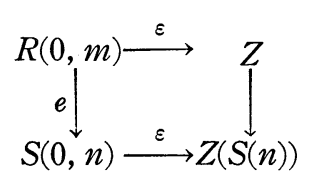

In terms of the decomposition formula, the boundary operator is expressed in the following form.

For $q=1$, we have

$$
\begin{aligned}
\partial\left[\alpha_{1}|\cdots| \alpha_{\iota}\right]= & \varepsilon \alpha_{1}\left[\alpha_{2}|\cdots| \alpha_{\iota}\right] \\
& +\sum_{i=1}^{\iota-1}(-1)^{i}\left[\alpha_{1}|\cdots| \alpha_{i} \alpha_{i+1}|\cdots| \alpha_{\iota}\right]+(-1)^{\imath} \varepsilon \alpha_{\iota}\left[\alpha_{1}|\cdots| \alpha_{\iota-1}\right]
\end{aligned}
$$

For $q>1$, we have

$$
\begin{aligned}
\partial\left[\alpha_{1}|\cdots| \alpha_{\iota}\right]=\sum_{i=1}^{\iota}(-1)^{\left|\alpha_{1}\right|+\cdots+\left|\alpha_{i-1}\right|+i}\left[\alpha_{1}|\cdots| \partial \alpha_{i}|\cdots| \alpha_{\iota}\right] & \\
& +\sum_{i=1}^{\iota-1}(-1)^{\left|\alpha_{1}\right|+\cdots+\left|\alpha_{i}\right|+i}\left[\alpha_{1}|\cdots| \alpha_{i} \alpha_{i+1}|\cdots| \alpha_{\iota}\right] .
\end{aligned}
$$

Consequently we obtain

$$
S(q, n) \underset{S(n)}{\otimes} Z \cong \bar{B}^{(q-1)}\left(K(N, 1)_{N}\right)(n)
$$

where $\bar{B}^{(q-1)}$ denotes the $(q-1)$-fold iterated bar construction.

\section{§3. A projective resolution $W(n, \pm)$}

1. The definition of $W(n, \pm)$

Let us consider toe subset of $R(q, n)$ consisting of the cells $\alpha$ written in the form $\left(r_{1}, \cdots, r_{n}\right)$ such that $0<r_{\nu} \leqq q$ for $1 \leqq \nu \leqq n$ and $r_{1}=q$. As easily seen these cells generates a $S(n)$-free cochain submodule of the dual $R^{*}(q, n)$ of $R(q, n)$. Given a dual $\alpha^{*} \in R^{*}(q, n)$ of a cell $\alpha \in R(q, n)$ of the form $\left(r_{1}, \cdots, r_{n}\right)$ with the properties mentioned above, we associate to $\alpha^{*}$ a sequence $\hat{\alpha}=\left\langle\rho_{1}, \cdots, \rho_{n}\right\rangle$ such that $0 \leqq \rho_{\nu}<q$ for $1 \leqq \nu \leqq n$ and $\rho_{1}=0$ where $\rho_{\nu}$ is defined to be $q-r_{\nu}$. Now we define $W(n, q)$ to be the $S(n)$-free chain module with the symbols $a=$ $\left\langle\rho_{1}, \cdots, \rho_{n}\right\rangle$ as its $S(n)$-base for which we define

$$
\operatorname{dim}\left\langle\rho_{1}, \cdots, \rho_{n}\right\rangle=\sum_{\nu=1}^{n} \rho_{\nu} .
$$

The incidence number is given by

$$
[\hat{\beta}: x \hat{\alpha}]=\left[\alpha: x^{-1} \beta\right]
$$

with an arbitrary $x \in S(n)$.

Suppose given a symbol $a=\left\langle\rho_{1}, \cdots, \rho_{n}\right\rangle \in W(n, q)$ and an integer $\rho$ such that 
$0 \leqq \rho<q$. Then we define a monotone increasing sequence

$$
\hat{\nu}(\rho, 1), \cdots, \hat{\nu}(\rho, i), \cdots, \hat{\nu}(\rho, \hat{\imath}(\rho))
$$

to be the set of $\nu^{\prime} s$ satisfying $\rho_{\nu} \leqq \rho$. Clearly we have $\hat{\nu}(\rho, i)=\nu(q-\rho, i)$, $\hat{\nu}(\rho, i+1)-\hat{\nu}(\rho, i)=n(q-\rho, i)$ and so on.

Let $\pi$ be a permutation of the form

$$
\pi=\left(\begin{array}{ccc}
1, & \cdots, & \hat{\imath}(\rho) \\
\pi(1), & \cdots, \pi(\hat{\imath}(\rho))
\end{array}\right)
$$

Then we define a permutation $\hat{x}_{\hat{\alpha}}(\rho, \pi) \in S(n)$ to be $x_{\alpha}(q-\rho, \pi)$.

In the following, we shall be mainly concerned with the sequences

$$
\hat{\nu}(0,1), \cdots, \hat{\nu}(0, \iota)
$$

where we have $\hat{\nu}(0, i+1)-\hat{\nu}(0, i)=n_{i}$. We put

$$
a_{i}=\left\langle 0, \rho_{\hat{\nu}(0, i)+1}-1, \cdots, \rho_{\hat{\nu}(0, i+1)-1}-1\right\rangle .
$$

Then $a_{i}$ determines a generator of $W\left(n_{i}, q-1\right)$. Thus we have a decomposition

$$
a=\left\langle a_{1}, \cdots, a_{\iota}\right\rangle
$$

and an isomorphism

$$
W(n, q) \cong \sum_{n_{1}+\cdots+n_{\iota}=n} Z(S(n)) \otimes_{S\left(n_{1}\right) \times \cdots \times S\left(n_{\iota}\right)}\left(W\left(n_{1}, q-1\right) \otimes \cdots \otimes W\left(n_{\iota}, q-1\right)\right) .
$$

Let $(l, m)$ be a pair of nonnegative integers such that $l+m=n$. Then we define an $S(n)$-equivariant chain homomorphism

$$
T(l, m): W(n, q) \longrightarrow Z(S(n)) \otimes_{S(l) \times S(m)}(W(l, q) \otimes W(m, q))
$$

which will be called the transfer. Let $\pi \in S(\iota)$ be a permutation

$$
\pi=\left(\begin{array}{ccc}
1, & \cdots, c \\
\pi(1), \cdots, \pi(\iota)
\end{array}\right)
$$

such that

$$
\begin{aligned}
\pi(1) & <\cdots<\pi(i) \\
\pi(i+1) & <\cdots<\pi(\ell)
\end{aligned}
$$

for some $i$ satisfying the requirement

$$
\begin{gathered}
\sum_{j=1}^{i} n_{\pi(j)}=l \\
\sum_{j=i+1}^{i} n_{\pi(j)}=m
\end{gathered}
$$

Then we define

$$
\hat{\varepsilon}_{a}(0, \pi)=\left\{\begin{array}{lr}
(-1)^{\Sigma\left(\left|a_{j}\right|+1\right)\left(\left|a_{k}\right|+1\right)} & \text { if } q \equiv 0 \bmod 2 \\
(-1)^{\Sigma\left(\left|a_{j}\right|+n_{j}+1\right)\left(\left|a_{k}\right|+n_{k}+1\right)} & \text { if } q \equiv 1 \bmod 2,
\end{array}\right.
$$

where the summation is extended over the set of couples $(j, k)$ such that $1 \leqq j \leqq i, i+1 \leqq k \leqq \iota$ and $\pi(j)>\pi(k)$.

Now the transfer is given as follows: 


$$
T(l, m)\left\langle a_{1}, \cdots, a_{\imath}\right\rangle=\sum \hat{\varepsilon}_{a}(0, \pi) \hat{x}_{a}(0, \pi)\left\langle a_{\pi(1)}, \cdots, a_{\pi(i)}\right\rangle \otimes\left\langle a_{\pi(i+1)}, \cdots, a_{\pi(\iota)}\right\rangle
$$

where $a=\left\langle a_{1}, \cdots, a_{\iota}\right\rangle$.

Summarizing the results, we have

$$
\operatorname{dim}\left\langle a_{1}, \cdots, a_{\iota}\right\rangle=\sum_{i=1}^{\iota} \operatorname{dim} a_{i}+n-\iota
$$

and the boundary formula is given as follows :

In case $q \equiv 0 \bmod 2$, we have

$$
\begin{aligned}
\partial\left\langle a_{1}, \cdots, a_{\imath}\right\rangle & =\sum_{i=1}^{\iota}(-1)^{\left|a_{1}\right|+\cdots+\left|a_{i-1}\right|+i}\left\langle a_{1}, \cdots, \partial a_{i}, \cdots, a_{\iota}\right\rangle \\
& +\sum_{i=1}^{\iota}(-1)^{\left|a_{1}\right|+\cdots+\left|a_{i}\right|+i}\left\langle a_{1}, \cdots, T a_{i}, \cdots, a_{\imath}\right\rangle
\end{aligned}
$$

and in case $q \equiv 1 \bmod 2$, we have

$$
\begin{aligned}
\partial\left\langle a_{1}, \cdots, a_{\iota}\right\rangle & =\sum_{i=1}^{\iota}(-1)^{\left|a_{1}\right|+n_{1}+\cdots+\left|a_{i-1}\right|+n_{i-1}+i}\left\langle a_{1}, \cdots, \partial a_{i}, \cdots, a_{\iota}\right\rangle \\
& +\sum_{i=1}^{\iota}(-1)^{\left|a_{1}\right|+n_{1}+\cdots+\left|a_{i}\right|+n_{i}+i}\left\langle a_{1}, \cdots, T a_{i}, \cdots, a_{\iota}\right\rangle
\end{aligned}
$$

where $T$ denotes $\sum_{l+m=n_{i}} T(l, m)$.

The above formulas show that $W(n, q)$ is naturally imbedded in $W(n, r)$ if $r-q$ is an even positive integer. Hence $W(n, 2 q)$ converges to a free projective resolution of $Z$ over the group ring $Z(S(n))$ when $q$ tending to $\infty$. The limit $\lim _{q \rightarrow \infty} W(n, 2 q)$ will be denoted by $W(n,+)$. In the same way, $W(n, 2 q+1)$ converges to a free projective resolution of $Z_{-}$over the group ring $Z(S(n))$ where $Z_{-}$ denotes a module of integers on which $S(n)$ operates by sign changing operation. The limit $\lim _{q \rightarrow \infty} W(n, 2 q+1)$ will be denoted by $W(n,-)$.

The formula

$$
W(n, q) \cong \sum Z(S(n)){ }_{S\left(n_{1}\right) \times \cdots \times S\left(n_{\iota}\right)}\left(W\left(n_{1}, q-1\right) \otimes \cdots \otimes W\left(n_{\iota}, q-1\right)\right.
$$

implies the decomposition formulae

$$
\begin{array}{ll}
W(n,+) \cong \sum Z\left(S \left(n_{S\left(n_{1}\right) \times \cdots \times S\left(n_{\iota}\right)}\right.\right. & \left(W\left(n_{1},-\right) \otimes \cdots \otimes W\left(n_{\iota},-\right)\right) \\
W(n,-) \cong \sum Z(S(n)) & \otimes_{S\left(n_{1}\right) \times \cdots \times S\left(n_{\iota}\right)}
\end{array}
$$

and the corresponding boundary formulae as stated before.

In the following, we shall use the notations $W^{+}(n, \pm)$ or $W^{+}(n)$ or simply $W(n)$ to indicate $W(n, \pm) \otimes Z_{ \pm}$.

2. The duality between $W(n, q)$ and ${ }_{n} A^{\prime}(N, q)$

Let $\alpha$ denote the generator of the semigroup of nonnegative integers $N$ and $s$ be an odd positive integers. Now we denote by ${ }^{(s)} \alpha$ the $s$-fold suspension of $\alpha$ in the sense of bar-construction. Then we consider the $(q-s)$-fold bar construction $\bar{B}^{(q-s)}\left(E{ }^{(s)} \alpha\right)$ ) of the exterior algebra $E\left({ }^{(s)} \alpha\right)$ with one generator ${ }^{(s)} \alpha$ in dimension $s$, which will be denoted by $A^{(s)}(N, q)$. Clearly $A^{(s)}(N, q)$ is a d.g.a. subalgebra of $A(N, q)$, so we use the notation $A^{(s)}(N, q, n)$ to indicate the submodule $A^{(s)}(N, q)(n)$ of $A^{(s)}(N, q)$ generated by the elements of multiplicity 
$\leqq n$. Here we should note that, in the special case $s=1$, the natural injection: $A^{\prime}(N, q, n) \rightarrow A(N, q, n)$ induces a chain homotopy equivalence. Furthemore, we shall denote by $W^{+}(n, q)$ the submodule $W(n, q) \otimes Z_{ \pm}$of $W^{+}(n, \pm)$ accordingly $(-1)^{q}= \pm 1$.

Under these conventions, we get isomorphisms

$$
W^{+}(n, q) \underset{S(n)}{\otimes}\left(\otimes_{n} M(Z, q)_{N}^{*}\right) \cong{ }_{n} A^{\prime}(N, q)^{*}
$$

such that the following diagram is commutative

$$
W^{+}(n, q) \underset{S(n)}{\otimes}\left({ }_{n} A^{\prime}(N, q)^{*} M(Z, q)_{N}^{*}\right) \longrightarrow W^{+}(n, r) \underset{S(n)}{\otimes}\left({\underset{n}{n}}_{n} M(Z, q)_{N}^{*}\right)
$$

where $i^{\prime}$ maps ${ }_{n} A^{\prime}(N, q)^{*}$ isomorphically onto ${ }_{n} A^{(r-q+1)}(N, r)^{*}$ for $r>q$.

$\S 4$. Cell decomposition of the $\boldsymbol{n}$-th product of miscellaneous spaces.

Let $H^{q}$ be a half space of $R^{q}$ defined by

$$
H^{q}=\left\{\boldsymbol{a} \in R^{q} \mid a^{1} \leqq 0\right\} .
$$

Clearly the cell decomposition $R(q, n)$ of $\times R^{q}$ induces a cell decomposition of $\times H^{q}$ which will be denoted by $H(q, n)$. As before we arrange the set of $\nu^{l} \mathrm{~s}$ with $r_{\nu}^{n}=q$ in the monotone increasing sequence

$$
\nu(q, 1), \cdots, \nu(q, \iota)
$$

and we put $\nu(q, i+1)-\nu(q, i)=n_{i}$. Then we have a unique decomposition of a cell $\alpha \in H(q, n)$

$$
\alpha=\alpha_{0}\left[\alpha_{1}|\cdots| \alpha_{\iota}\right]
$$

with $\alpha_{i} \in R\left(q-1, n_{i}\right)$ for $0 \leqq i \leqq \imath$ and hence an isomorphism

$$
H(q, n) \cong \sum_{n_{0}+\cdots+n_{\iota}=n} Z(S(n))_{S\left(n_{0}\right) \times \cdots \times S\left(n_{\iota}\right)}\left(R\left(q-1, n_{0}\right) \otimes \cdots \otimes R\left(q-1, n_{\iota}\right)\right)
$$

where

$$
\begin{gathered}
\operatorname{dim} \alpha=\sum_{i=1}^{\iota} \operatorname{dim} \alpha_{i}+\iota \\
\partial\left(\alpha_{0}\left[a_{1}|\cdots| \alpha_{\iota}\right]\right)=\partial \alpha_{0}\left[\alpha_{1}|\cdots| \alpha_{\iota}\right]+\alpha_{0} \alpha_{1}\left[\alpha_{2}|\cdots| \alpha_{\iota}\right] \\
+\sum_{i=1}^{\iota}(-1)^{|\alpha|+\cdots+\left|\alpha_{i-1}\right|+i} \alpha_{0}\left[\alpha_{1}|\cdots| \partial \alpha_{i} \mid \cdots \alpha_{\iota}\right] \\
+\sum_{i=1}^{t-1}(-1)^{\left|\alpha_{0}\right|+\cdots+\left|\alpha_{i}\right|+i} \alpha_{0}\left[\alpha_{1}|\cdots| \alpha_{i} \alpha_{i+1} \mid \alpha_{i}\right] .
\end{gathered}
$$

Consequently we have

$$
H(q, n) \otimes_{S(n)} Z \cong{ }_{n} B(N, q) .
$$

Again we identify the $q$-dimensional disk $D^{q}$ with the one-point-compactification of $H^{q}$ in which $\partial D^{q}$ is naturally identified with the compactification of $R^{q-1}$ 
which is the suspace of $R^{q}$ defined by $a^{1}=0$.

Now the decomposition $H(q, n)$ of $\times H^{q}$ is extended to a cell decomposition $D(q, n)$ of $\underset{n}{\times} D^{q}$. The following isomorphisms will be evident.

$$
\begin{aligned}
& D(q, n) \cong \sum_{m=0}^{n} Z(S(n)){\underset{S(m) \times S(n-m)}{\otimes}}_{\otimes} H(q, m) \\
& D(q, n) \bigotimes_{S(n)} Z \cong B \bar{B}^{(q-2)}\left(K(N, 1)_{N}\right)(n) .
\end{aligned}
$$

Finally we consider the map $\theta: S^{q-1} \rightarrow R^{q-1}$ defined by $f(\boldsymbol{a})=\tan \left(\theta\left(\tan ^{-1}|\boldsymbol{a}|\right)\right) \cdot \boldsymbol{a}$. We consider a space $D_{\theta}^{q}=S^{q-1} \cup_{\theta} D^{q}$. The decomposition $D(q, n)$ of $\times D^{q}$ induces a cell decomposition of $\times D_{\theta}^{q}$ which will be denoted by $D_{\theta}(q, n)$. " Among the results obtained for $D_{\theta}(q, n)$, the following is important:

$$
D_{\theta}(q, n) \underset{S(n)}{\otimes} Z \cong\left(\bar{B}^{(q-2)}\left(K(N, 1)_{N}\right) \underset{\bar{T}_{\theta}}{\otimes} \bar{B}^{(q-1)}\left(K(N, 1)_{N}\right)\right)(n)
$$

\section{§. Supplements}

All the results stated in this section are the analogues for the barconstruction of the corresponding results already obtained for the $W$-construction in Chap. 1. They can be proved in the same way as in Chap. 1, so we will omit the proof.

The arguments developed in $\S 5$ in the Chap. 1 can be applied to the celldecompositions $R(q, n)$ and $H(q+1, n)$ instead of $\times{ }_{1} K(N, q)$ and $\times{ }_{1} W(N, q)$ respectively and we obtain the cochain homomorphisms

and

$$
{ }_{n} \psi(Z, q):{ }_{n} A(N, q)^{*} \longrightarrow W(n) \underset{S(n)}{\otimes}\left(\bigotimes_{n} M(Z, q)_{N}^{*}\right)
$$

$$
{ }_{n} \psi(0, q):{ }_{n} B(N, q)^{*} \longrightarrow W(n) \underset{S(n)}{\otimes}\left(\otimes_{n} M(0, q)_{N}^{*}\right) .
$$

Clearly ${ }_{n} \psi(0, q)$ gives rise to cochain homomorphisms

$$
{ }_{n} \psi\left(Z_{\theta}, q\right):{ }_{n} A_{\theta}(N, q)^{*} \longrightarrow W(n) \underset{S(n)}{\otimes}\left(\bigotimes_{n} M\left(Z_{\theta}, q\right)_{N}^{*}\right) .
$$

These homomorphisms are abbreviated as $\phi$ in the case we have no fear of confusion.

Let $G^{m}$ be the cochain submodule of ${ }_{n} A_{\theta}(N, q)^{*}$ generated by the dual of the generators of $\mu_{B} \geqq m$. Put $G^{n-m, m}={ }_{n} A_{\theta}(N, q)^{*} \cap G^{m}$ and $E_{0}^{l, m}=G^{l, m} / G^{l-1, m+1}$.

Then we have

Lemma 2.1.

Furthermore we obtain

$$
\phi\left(G^{l, m}\right) \subset C^{l, m} .
$$

Proposition 2.2. The following diagram is commutative

$$
\begin{aligned}
& \begin{array}{ccc}
G^{l, m} / G^{l-1, m+1} & \stackrel{\psi}{\longrightarrow} & C^{l, m} / C^{l-1, m+1} \\
i \uparrow & & \uparrow k
\end{array} \\
& { }_{\imath} A(N, q)^{*} \otimes_{m} A(N, q+1)^{*} \stackrel{\psi \otimes \psi}{\longrightarrow} W(l) \otimes \otimes_{S(l)}\left(\bigotimes_{l} M(Z, q)_{N}^{*}\right) \otimes W(m) \underset{S(m)}{\otimes}\left(\bigotimes_{m} M(Z, q+1)_{N}^{*}\right)
\end{aligned}
$$


where $i$ and $k$ denote the natural injections.

Let us denote by $\phi_{0}^{l, m}: E_{0}^{l, m}(G) \rightarrow E_{0}^{l, m}(C)$ the cochain homomorphisms induced from $\phi$. Then Proposition 2.2 can be written in the form

$$
\phi_{0}^{l, m}=k\left({ }_{l} \psi(Z, q) \otimes_{m} \psi(Z, q+1)\right) i^{-1} .
$$

Using the suspension of the bar-construction, we can construct the cochain homomorphisms

$$
{ }_{m} \varphi(Z, q): W(n) \underset{S(n)}{\otimes}\left(\otimes_{n} M(Z, q)_{N}^{*}\right) \longrightarrow{ }_{n} A(N, q)^{*}
$$

and

$$
{ }_{n} \varphi(0, q): W(n) \underset{S(n)}{\otimes}\left(\otimes_{n} M(0, q)_{N}^{*}\right) \longrightarrow{ }_{n} B(N, q)^{*}
$$

inductively. Again ${ }_{n} \varphi(0, q)$ gives rise to cochain homomorphisms

$$
{ }_{n} \varphi\left(Z_{\theta}, q\right): W(n) \underset{S(n)}{\otimes}\left(\otimes_{n} M\left(Z_{\theta}, q\right)_{N}^{*}\right) \longrightarrow{ }_{n} A_{\theta}(N, q)^{*}
$$

We shall abbreviate ${ }_{n} \varphi\left(Z_{\theta}, q\right)$ simply as $\varphi$. Then we have

Lemma 2.3.

$$
\varphi\left(C^{l, m}\right) \subset G^{l, m}
$$

Proposition 2.4. The following diagram is commutative

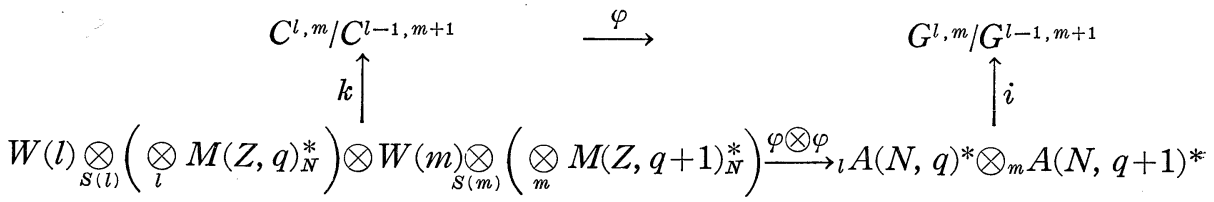

or equivalently

$$
\varphi_{0}^{l, m}=i\left({ }_{l} \varphi(Z, q) \otimes_{m} \varphi(Z, q+1)\right) k^{-1}
$$

where $\varphi_{0}^{l, m}: E_{0}^{l, m}(C) \rightarrow E_{0}^{l, m}(G)$ denotes the induced homomorphism.

Consequently we have

THEOREM 2.5. Let

$$
{ }_{n} \psi\left(Z_{\theta}, q\right):{ }_{n} A_{\theta}(N, q)^{*} \longrightarrow W(n) \underset{S(n)}{\otimes}\left(\underset{n}{\otimes} M\left(Z_{\theta}, q\right)_{N}^{*}\right)
$$

and

$$
{ }_{n} \varphi\left(Z_{\theta}, q\right): W(n) \underset{S(n)}{\otimes}\left(\otimes_{n} M\left(Z_{\theta}, q\right)_{N}^{*}\right) \longrightarrow{ }_{n} A_{\theta}(N, q)^{*}
$$

be the cochain homomorphisms defined as above. Then the composition

$$
{ }_{n} \varphi\left(Z_{\theta}, q\right) \cdot{ }_{n} \psi\left(Z_{\theta}, q\right):{ }_{n} A_{\theta}(N, q)^{*} \longrightarrow{ }_{n} A_{\theta}(N, q)^{*}
$$

gives a cochain equivalence.

Corollary 2.6. Under the same notations as above, we have 


$$
{ }_{n} \varphi(Z, q)_{n} \psi(Z, q) \simeq i d:{ }_{n} A(N, q)^{*} \longrightarrow{ }_{n} A(N, q)^{*}
$$

Proof. Since ${ }_{n} \psi(Z, q)$ induces an injection of the cohomology, we only need to verify

$$
{ }_{n} \psi(Z, q)_{n} \varphi(Z, q)_{n} \psi(Z, q) \simeq{ }_{n} \psi(Z, q)
$$

Let us introduce an $S(n)$-equivariant simplicial subdivision $K(q, n)$ of the $C W$-complex $D(q, n)$ whose underlying space is $\underset{n}{\times} D^{q}$. The induced triangulation of the boundary of $\underset{n}{\times} D^{q}$ will be denoted by ${ }^{n} \dot{K}(q, n)$. Let $K$ be a locally finite free $F D$-submodule of $K\left(D^{q}\right) / K\left(S^{q}\right)$ with sufficiently many simplices. Then we have an $S(n)$-equivariant cochain equivalence

$$
i^{\prime}:(\underset{n}{\times} K) * \longrightarrow(K(q, n) / \dot{K}(q, n))^{*}
$$

and the dual of the $S(n)$-equivariant subdivision

Finally we define

$$
j^{\prime}:\left(K(q, n)_{i}^{\prime} \dot{K}(q, n)\right)^{*} \longrightarrow R(q, n)^{*}
$$

$$
\varphi_{0}^{\prime}: W(n) \otimes\left(\underset{n}{\otimes} M(Z, q)_{N}^{*}\right) \longrightarrow(\underset{n}{\times} K)^{*}
$$

to be a Steenrod operation. Clearly all the homomorphisms $\varphi_{0}^{\prime}, i, j^{\prime}$ are $S(n)$ equivariant.

Let us denote by $\varphi_{0}, i$ and $j$ the quotient homomorphism $\varphi_{0}^{\prime} / S(n), i^{\prime} / S(n)$ and $j^{\prime} / S(n)$ respectively. Then the same argument as in Lemma $1.33 \S 5$, Chap. I can be applied to yield a homotopy ${ }_{n} \psi(Z, q) j i \varphi_{0}{ }_{n} \psi(Z, q)$. On the other hand, we have $j i \varphi_{0} \simeq_{n} \varphi(Z, q)$. This concludes

$$
{ }_{n} \psi(Z, q)_{n} \varphi(Z, q)_{n} \psi(Z, q) \simeq_{n} \psi(Z, q)
$$

REMARK. We remark here the existence of a cochain homomorphism

satisfying $\mu \psi \leqq \mu$ and $\varphi \phi \simeq i d$.

$$
\phi: A(N, q, n)^{*} \longrightarrow W(n) \underset{S(n)}{\otimes}\left(\underset{n}{\otimes} L(Z, q)_{N}^{*}\right)
$$

In the same way as before, $\phi$ can be extended to a cochain homomorphism

$$
\tilde{\psi}: B(N, q, n)^{*} \longrightarrow W(n) \underset{S(n)}{\otimes}\left(\otimes_{n} L(0, q)_{N}^{*}\right) .
$$

\section{Chapter 3. The secondary cohomology operation.}

In this chapter, we introduce a combinatorial definition of the secondary cohomology operation. Although the argument is here restricted to the secondary case, our method can be applied to provide a combinatorial definition of the cohomology operation of higher orders. Moreover it should be remarked that all the results contained in $\S 3$ have algebraic analogue, for example, we cen define secondary cohomology operations on a resolution over a Hopf algebra.

\section{§1. A twisted cartesian product and a twisted tensor product.}

1. Twisted cartesian product.

Let $K$ be an $F D$-algebra and $L$ be an $F D$-coalgebra i.e. an $F D$-module with 
diagonal $d: L \rightarrow L \times L$. By a twisting function $t$, we mean a homomorphism $t: L \rightarrow K$ satisfying the following conditions :
i) $t L_{r} \subset K_{r-1}$
ii) $\partial_{0} t \tau=m\left(t^{-1} \partial_{0} \times t \partial_{1}\right) d \tau$
iii) $\partial_{i} t \tau=t \partial_{i+1} \tau$
iv) $s_{i} t \tau=t s_{i+1} \tau$
v) $t s_{0} \tau=1$

for $i>0$

for $i \geqq 0$

where $m: K \times K \rightarrow K$ denotes a multiplication.

Then a twisted cartesian product $K \times \underset{t}{ } L$ of $K$ and $L$ with twisting function $t$ is an $F D$-module defined as follows :

i) $(K \times L){ }_{t} \cong(K \times L)_{p}$

ii) $\partial_{0}(\sigma \times \tau)=\partial_{0} \sigma \cdot\left(t \times \partial_{0}\right) d \tau$ $\partial_{i}(\sigma \times \tau)=\partial_{i} \sigma \times \partial_{i} \tau$ for $i>0$

iii) $s_{i}(\sigma \times \tau)=s_{i} \sigma \times s_{i} \tau$ with $\sigma \in K$ and $\tau \in L$.

Suppose given a twisted cartesian product $K \times L$ and an $F D$-homomorphism $f: L^{\prime} \rightarrow L$ between free $F D$-coalgebras not necessarily transforming a simplex into a simplex. Clearly the homomorpnism if satisfies the conditions of the twisting function, so we can form an induced product $f^{-1}(K \times L)$ to be $K \times L^{\prime}$.

For example, we take an $F D$-algebra $K(\Pi, q)$ and an $F D$-coalgebra $K(\Pi, q+1)$. Then an arbitrary $r$-simplex $\tau$ of $K(\Pi, q+1)$ can be decomposed into the product $\tau=\left\langle\sigma_{r-1}, \cdots, \sigma_{0}\right\rangle$ of $i$-simplices $\sigma_{i}$ of $K(\Pi, q)$. Now we set $u(\tau)=\sigma_{r-1}$. Obviously $u$ defines a twisting function $u: K(\Pi, q+1) \rightarrow K(\Pi, q)$ such that the resulting twisted cartesian product $K(\Pi, q) \times K(\Pi, q+1)$ is $W(\Pi, q)$.

We choose an arbitrary $r$-simplex $\tau$ of a coalgebra $L$. Then we have a characteristic homomorphism $\hat{\tau}: \Delta(r) \rightarrow L$ such that $\hat{\tau}(0,1, \cdots, r)=\tau$. Given an $(q+1)$-cocycle $k \in Z^{q+1}(L ; I I)$, we have a $(q+1)$-cycle $\hat{\tau}^{\sharp}(k) \in Z^{q+1}(\Delta(r) ; \Pi)$. By the definition of the Eilenberg-MacLane complexes, $\hat{\tau}^{\sharp}(k)$ can be considered as a $r$-simplex of $K(\Pi, q+1)$. Thus we have an $F D$-homomorphism $T(k): L \rightarrow K(\Pi, q+1)$. The induced product $K(\Pi, q) \underset{u T(k)}{\times} L$ will be denoted simply by $K(\Pi, q) \underset{k}{\times} L$ or $K(\Pi, q) \underset{T}{\times} L$.

On the other hand, we know that $K(N, q)$ is $F D$-homotopy equivalent to $K(Z, q)$. Hence a $(q+1)$-cocycle $k \in Z^{q+1}(L ; Z)$ provides us with an $F D$-homomorphism $T(k): L \rightarrow K(N, q+1)$ and we obtain an induced product $K(N, q) \underset{u T(k)}{\times} L$ which will be denoted by $K(N, q) \underset{T}{\times} L$.

2. Twisted tensor products.

Let $A$ be a d.g.a. algebra and $B$ a d.g.a. coalgebra that is a d.g.a. module with diagonal $d: B \rightarrow B \otimes B$. By a twisting cochain $\varphi$, we mean a homomorphism $\varphi: B \rightarrow A$ satisfying the following conditions.

i) $\varphi B_{r} \subset A_{r-1}$ for $r>0$ and $\varphi B_{0}=0$

ii) $\varepsilon \varphi=0$

iii) $\partial \varphi+\varphi \partial=(\varphi \omega) \cup \varphi$

where $\varepsilon$ denotes an augmentation and $\omega: A \rightarrow A$ denotes an involution defined by $\omega \mid A_{r}=(-1)^{r}$.

Then a twisted tensor product $A \underset{\varphi}{\otimes} B$ of $A$ and $B$ with twisting cochain $\varphi$ 
is a d.g.a. module defined as follows.

i) $(A \otimes B)_{r} \cong(A \otimes B)_{r}$

ii) $\partial(1 \otimes b)=1 \otimes \partial b+(1 \otimes b) \cap \varphi$ $\partial(a \otimes b)=\partial a \otimes b+(-1)^{|a|} a \cdot \partial(1 \otimes b)$

where $a$ and $b$ denote the elements of $A$ and $B$ respectively and $\cap \varphi$ denotes the composition of the sequence of homomorphisms

$$
\cap \varphi: A \otimes B \stackrel{1 \otimes d}{\longrightarrow} A \otimes B \otimes B \stackrel{1 \otimes \varphi \otimes 1}{\longrightarrow} A \otimes A \otimes B \stackrel{m \otimes 1}{\longrightarrow} A \otimes B .
$$

Suppose given a twisted tensor product $A \bigotimes_{\varphi} B$ and a d.g.a. homomorphism $f: B^{\prime} \rightarrow B$ between d.g.a. coalgebras. In the same way as before, the induced product $f^{-1}\left(A \underset{\varphi}{\left.\bigotimes_{0} B\right)}\right.$ is defined to be $A \bigotimes_{\varphi f} B^{\prime}$.

We consider a d.g.a. algebra $A(\Pi, \varphi, q)$ and d.g.a. coalgebar $A(\Pi, q+1)$. Take a generator of $A(\Pi, q+1)$ which can be decomposed into a product

$$
b=\left[a_{1}|\cdots| a_{\nu}\right]
$$

of generators $a_{i}$ of $A(\Pi, q)$. Now we set $u(b)=\delta_{1}^{\nu} a_{1}$. Then $u$ defines a twisting cochain $u: A(\Pi, q+1) \rightarrow A(\Pi, q)$ such that the resulting twisted tensor product $A(\Pi, q) \otimes_{u} A(\Pi, q+1)$ is the bar-construction $B(\Pi, q)$ of $A(\Pi, q)$.

.Given an arbitrary $(q+1)$-cocycle $k \in Z^{q+1}(B ; Z)$ of a d.g.a. coalgebra $B$. We can define a d.g.a. homomorphism $\varphi(k): B \rightarrow A(N, q+1)$ which is equivalent to $T(k)$. We obtain an induced product $A(N, q) \underset{u \varphi(k)}{\otimes} B$ which will be denoted by $A(N, q) \underset{\varphi}{\otimes} B$.

For the details, see Brown [3], Szczarba [21].

3. The dual of twisted tensor products.

For later use, we shall state some remarks concerning the duals of twisted tensor products.

Let $A, B$ and $\varphi$ be as before. Furthermore we assume now $A$ and $B$ are free and locally finite. Then the dual homomorphism $\varphi^{*}: A^{*} \rightarrow B^{*}$ of $\varphi$ satisfies the following conditions :

$$
\begin{aligned}
\text { i)* } & \varphi^{*} A_{r}^{*} \subset B_{r+1}^{*} \\
\text { ii)* } & \varphi^{*} \varepsilon^{*}=0 \\
\text { iii)* } & \varphi^{*} \delta+\delta \varphi^{*}=\left(\omega \varphi^{*}\right) \vee \varphi^{*}
\end{aligned}
$$

where $\varphi^{*} \omega \vee \varphi^{*}$ denotes the composition of a sequence of the homomorphisms

$$
\varphi^{*} \omega \vee \varphi^{*}: A^{*} \stackrel{m^{*}}{\longrightarrow} A^{*} \otimes A^{*} \stackrel{\varphi^{*} \omega \otimes \varphi^{*}}{\longrightarrow} B^{*} \otimes B^{*} \stackrel{d^{*}}{\longrightarrow} B^{*} .
$$

Now remark that $B^{*}$ has a product $d^{*}$ and $A^{*}$ has a coproduct $m^{*}$. Then $\varphi^{*}: A^{*} \rightarrow B^{*}$ defines an analogue of a twisting cochain and an analogue of a twisted tensor product $A^{*} \bigotimes_{\varphi^{*}} B^{*}$ with a twisting cochain $\varphi^{*}$ is defined to be $(A \underset{\varphi}{\otimes} B)^{*}$. Clearly we have the following relations

i) $\left(A^{*} \otimes B^{*}\right)^{r} \cong\left(A^{*} \otimes B^{*}\right)^{r}$

ii) $\delta(\alpha \otimes 1)=\delta \alpha \otimes 1+(\alpha \otimes 1) \wedge \varphi^{*}$ $\delta(\alpha \otimes \beta)=\delta(\alpha \otimes 1) \beta+(-1)^{\mid \alpha !} \alpha \otimes \delta \beta$

where $\alpha$ and $\beta$ are the elements of $A^{*}$ and $B^{*}$ respectively and $\wedge \varphi^{*}$ denotes the composition of the sequence of homomorphisms

$$
\wedge \varphi^{*}: A^{*} \otimes B^{*} \stackrel{m^{*} \otimes 1}{\longrightarrow} A^{*} \otimes A^{*} \otimes B^{*} \stackrel{\omega \otimes \varphi^{*} \otimes 1}{\longrightarrow} A^{*} \otimes B^{*} \otimes B^{*} \stackrel{1 \otimes d^{*}}{\longrightarrow} A^{*} \otimes B^{*} .
$$


We can formulate some results analogous to the case of original d.g.a. algebras. However they will not be used in the following discussion.

\section{§ 2. Universal examples.}

1. The secondary cohomology operations.

Throughout this section, we shall restrict our arguments to the category of Kan complexes (See Dold [5]).

Let $k$ be a $(q+1)$-cocycle of $K(Z, p)$. Then we can construct a twisted cartesian product $K(p, k, q)=K(Z, q) \times{ }_{k} K(Z, p)$. In the following discussion, we shall employ the term the universal examples for spaces of type $K(p, k, q)$ and the secondary cohomology operations in the strong sense for elements of the cohomology module of $K(p, k, q)$. Here we use the notation $\iota: K(Z, q) \rightarrow K(p, k, q)$ for the natural injection and $\pi: K(p, k, q) \rightarrow K(Z, p)$ for the natural projection and $m: K(Z, q) \times K(p, k, q) \rightarrow$ $K(p, k, q)$ for the fibre multiplication.

Now we suppose given a homomorphism $g: K \rightarrow K(Z, p)$ such that $g^{*} k \sim 0$. Then we can obtain a homomorphism $f: K \rightarrow K(p, k, q)$ satisfying $\pi f=g$. We say that $f$ is a lift of $g$ or $g$ is lifted to $f$.

Corresponding to the well-known theorems concerning secondary cohomology operations in the stable range, we have the following propositions. They can be proved using the same argument as in the stable case. Hence the proof will be omitted here. (Cf. Peterson-Stein [15], Shimada-Yamanoshita [7])

Proposition 3.1 Let $f_{0}, f_{1}: K \rightarrow K(p, k, q)$ be two homomorphisms whose projections $\pi f_{0}$ and $\pi f_{1}$ are homotopic to each other. Then we have

$$
f_{1}^{*} \simeq d^{*}\left(f_{0}^{*} \otimes h^{*} \iota^{*}\right) m^{*}
$$

where $d: K \rightarrow K \otimes K$ denotes a diagonal and $h: K \rightarrow K(Z, q)$ denotes a homomorphism given in the following way. Let $v$ be a $q$-cochain of $K(p, k, q)$ defined by $v(\sigma \times \tau)=u(\sigma)$. where $u$ denotes a fundamental cocycle of the fibre $K(Z, q)$. Let $f_{1}^{\prime}: K \rightarrow K(p, k, q)$ be a homomorphism which is homotopic to $f_{1}$ and satisfies the relation $\pi f_{1}^{\prime}=\pi f_{0}$. Then we define $h$ by $h^{*} u=\left(f_{1}^{\prime}-f_{0}\right)^{*} v$.

Proposition 3.2 Let $i: K^{\prime} \rightarrow K$ be a homomorphism. Suppose given a homomorphism $g: K \rightarrow K(Z, p)$ with a lift $f: K \rightarrow K(p, k, q)$. Then a homomorphism $g i^{\prime}: K^{\prime} \rightarrow K(Z, p)$ can be lifted to $f i: K^{\prime} \rightarrow K(p, k, q)$.

2. Functional cohomology operations.

The results in this section will not be used in the sequel. However the functional cohomology operations are known to be closely related with the secondary cohomology operations, so we will give them here without proof.

Let $g: K \rightarrow K(Z, p)$ be a homomorphism such that $g * k \sim 0$. Then we obtain a lift $f: K \rightarrow K(p, k, q)$ of $g$. Let $i: K^{\prime} \rightarrow K$ be a homomorphism satisfying the relation $i^{*} g^{*} u \sim 0$, where $u$ is a fundamental cocycle of $K(Z, p)$. Then we have a homomorpism $f_{0}: K \rightarrow K(p, k, q)$ which is homotopic to $f$ and maps $K^{\prime}$ into $K(Z, q)$, there exists a homomorphism $f_{0}^{\prime}: K^{\prime} \rightarrow K(Z, q)$ satisfying i $f_{0}^{\prime}=f_{0} i$.

Proposition 3.3 Let $f_{0}^{\prime}, f_{1}^{\prime}: K^{\prime} \rightarrow K(Z, q)$ be homomorphisms so that we can obtain homomorphisms $f_{0}, f_{1}: K \rightarrow K(p, k, q)$ which is homotopic to each other and satisfy the relation $\mathrm{e} f_{\nu}^{\prime}=f_{\nu} i$. Then we have

$$
f_{1}^{*} \simeq d^{*}\left(f_{0}^{*} \otimes h^{*}\right) m^{*}
$$


where $d: K \rightarrow K \otimes K$ denotes a diagonal and $m^{\prime}: K(Z, q) \otimes K(Z, q) \rightarrow K(Z, q)$ is the multiplication and $h^{\prime}: K^{\prime} \rightarrow K(Z, q)$ is defined as follows. Let $\tilde{h}: K \rightarrow \Omega K(Z, p)$ be a homomorphism determined by a homotopy between $f_{0}$ and $f_{1}$. Now we define a homomorphism $h^{\prime}$ by $\left(h^{\prime}\right)^{*} v \sim-\tilde{h}^{*}(\omega k)$ where $\omega k$ denotes a cocycle of $\Omega K(Z, p)$ defined by $(\omega k)(\rho)=k(S \rho)$ while $S: \Omega K(Z, p) \rightarrow K(Z, p)$ denotes a suspension.

Proposition 3.4 Let $f_{0}, f_{1}: K \rightarrow K(p, k, q)$ be two homomorphisms whose projections $\pi f_{0}$ and $\pi f_{1}$ are homotopic to each other. Furthermore we assume that there exist homomorphisms $f_{0}^{\prime}, f_{1}^{\prime}: K^{\prime} \rightarrow K(Z, q)$ satisfying i $f_{\nu}^{\prime}=f_{\nu}$ for $\nu=0,1$. Then we have

$$
f_{1}^{* *} \simeq d^{*}\left(f_{0}^{*} \otimes h^{*} \otimes h^{*}\right) \mu^{\prime *}
$$

where $d: K \rightarrow K \otimes K \otimes K$ denotes a diagonal homomorphism $m^{\prime}: K(Z, q) \otimes K(Z, q) \otimes$ $K(Z, q) \rightarrow K(Z, q)$ denotes the multiplication, and $h, h^{\prime}$ are homomorphisms defined in Propositions 3.1 and 3.3.

\section{$\S 3$. The construction of $L(\kappa ; m, n)$}

In the sequel, we shall use the word "module" or "submodule" in the sense of cochain module or cochain submodule and "homomorphism" in the sense of cochain homomorphism. To simplify the notation, the $n$-th tensor product $\underset{n}{\otimes} M$ of a cochain module $M$ will be denoted by $M^{(n)}$.

Let $M(p)$ be a free module with a single generator $u$ in dimension $p$ and let $M(0)$ be a free module with generator 1 . Let $L(p)$ be a direct sum of $M(p)$, and $M(0)$.

Now we put

$$
N(m)=W(m) \underset{S(m)}{\otimes} L(p)^{(m)} .
$$

Let $m$ be a fixed nonnegative integer and let $\left(l^{\prime}, l^{\prime \prime}\right)$ be a pair of nonnegative integers such that $l^{\prime}+l^{\prime \prime}=m$. We shall define the submodule $N\left(l^{\prime}, l^{\prime \prime}\right)$ of $N(m)$ by

$$
N\left(l^{\prime}, l^{\prime \prime}\right)=W(m) \underset{S(m)}{\otimes} \sum x_{i}\left(M(p)^{\left(l^{\prime}\right)} \otimes M(0)^{\left(l^{\prime \prime}\right)}\right)
$$

where the summation is extended over the set of representatives $x_{i}$ of left cosets of $S(m)$ with respect to $S\left(l^{\prime}\right) \times S\left(l^{\prime \prime}\right)$.

Clearly we have an isomorphism

$$
N(m)=\sum N\left(l^{\prime}, l^{\prime \prime}\right)
$$

where the summation is extended over the set of couples $\left(l^{\prime}, l^{\prime \prime}\right)$ such that $l^{\prime}+l^{\prime \prime}=n$.

We shall denote by $I I$ and $P$ the groupe $S\left(l^{\prime}\right) \times S\left(l^{\prime \prime}\right)$ and $S(m)$ respectively. Then we can assume that $W(\Pi)=W\left(l^{\prime}\right) \otimes W\left(l^{\prime \prime}\right)$ is naturally imbedded in $W(P)=$ $W(m)$. Choosing one representative $x_{i}$ in each left coset of $P$ with respect to $\Pi$, an arbitrary free generator of

$$
N\left(l^{\prime}, l^{\prime \prime}\right)=W(m) \otimes \sum x_{i}\left(M(p)^{\left(l^{\prime}\right)} \otimes M(0)^{\left(l^{\prime \prime}\right)}\right)
$$

can be written uniquely in the form

$$
x_{i} y x_{j}^{-} \xi \otimes x_{i}\left(u^{\left(l^{\prime}\right)} \otimes 1^{\left(l^{\prime \prime}\right)}\right)
$$

with $y \in \Pi, \xi$ being a $P$-base of $W(P)$. Hence the natural injection induces an isomorphism 


$$
W(P) \bigotimes_{\Pi}\left(M(p)^{\left(l^{\prime}\right)} \otimes M(0)^{\left(l^{\prime \prime}\right)}\right) \rightarrow N\left(l^{\prime}, l^{\prime \prime}\right) .
$$

Now we consider the well-known homotopy retraction

$$
\rho: W(P) \otimes_{\Pi}\left(M(p)^{\left(l^{\prime}\right)} \otimes M(0)^{\left(l^{\prime \prime}\right)}\right) \rightarrow W(\Pi) \bigotimes_{\Pi}\left(M(p)^{\left(l^{\prime \prime}\right)} \otimes M(0)^{\left(l^{\prime \prime}\right)}\right) .
$$

Suppose

$$
\begin{aligned}
& x_{i}\left(u^{\left(l^{\prime}\right)} \otimes 1^{\left(l^{\prime \prime}\right)}\right) \\
= & u^{\left(l_{1}^{\prime}\right)} \otimes 1^{\left(l_{1}^{\prime \prime}\right)} \otimes \cdots \otimes u^{\left(l_{e}^{\prime}\right)} \otimes 1^{\left(l_{e}^{\prime \prime}\right)}
\end{aligned}
$$

with $l_{i}^{\prime}>0$ for $1<i \leqq e$ and $l_{i}^{\prime \prime}>0$ for $1 \leqq i<e$. Then the notation $W\left(x_{i}\right)$ will stand for

$$
W\left(x_{i}\right)=W\left(l_{1}^{\prime}\right) \otimes W(1)^{\left(l_{1}^{\prime \prime}\right)} \otimes \cdots \otimes W\left(l_{e}^{\prime}\right) \otimes W(1)^{\left(l_{e}^{\prime \prime}\right)} .
$$

Here we introduce a submodule $N\left(x_{i}\right)$ of $N(m)$ by

$$
N\left(x_{i}\right)=x_{i}^{-1} W\left(x_{i}\right) \underset{I I}{\otimes}\left(M(p)^{\left(l^{\prime}\right)} \otimes M(0)^{\left(l^{\prime \prime}\right)}\right) .
$$

Then we have an injection

$$
\rho_{i}: N\left(x_{i}\right) \rightarrow N(e)
$$

which can be assumed to be the restriction of $\rho$ on $N\left(x_{i}\right)$.

Finally we define a submodule $\bar{N}(m)$ of $N(m)$ by

$$
\bar{N}(m)=\sum_{l^{\prime}+l^{\prime \prime}=m}\left(W\left(l^{\prime}\right) \otimes W\left(l^{\prime \prime}\right)\right){ }_{S\left(l^{\prime}\right) \times S\left(l^{\prime \prime}\right)}\left(M(p)^{\left(l^{\prime \prime}\right)} \otimes M(0)^{\left(l^{\prime \prime}\right)}\right) .
$$

The results above obtained show that there exists a homotopy retraction

$$
\rho: N(m) \rightarrow \bar{N}(m)
$$

which can be obtained by extending $\rho$, so will be denoted by the same notation.

Let $N$ be a module and $\kappa$ be a $(q+1)$-cocycle of $N$. Let $M$ be a representative of $M(q)$ with generator $v$ in dimension $q$. Then a module $M+N$ is defined to be the direct sum of the modules $M$ and $N$ with additional coboundary operator defined by $\delta v=\kappa$.

Let $m$ and $n$ be a pair of nonnegative integers. Then the module $\bar{N}(m n)$ will be abbreviated simply as $N$.

Let $\kappa$ be $a(q+1)$-cocycle of $\bar{N}(m)$. Then we define a module $\widetilde{L}(\kappa ; m, n)$ by

$$
\widetilde{L}(\kappa ; m, n)=W(n) \otimes(M+N)^{(n)}
$$

which will be denoted by $\tilde{L}$ in the sequel.

For a fixed pair of integers $\left(n^{\prime}, n^{\prime \prime}\right)$ such that $n^{\prime}+n^{\prime \prime}=n$, we consider the two submodules $L^{\prime}$ and $L^{\prime \prime}$ of $\widetilde{L}$ defined as follows:

$$
\begin{aligned}
& L^{\prime}=\iota^{\prime}\left(W\left(n^{\prime}\right) \otimes W\left(n^{\prime \prime}\right)\right) \otimes(\underset{\kappa}{M+N})^{\left(n^{\prime}\right)} \otimes \bar{N}(m)^{\left(n^{\prime \prime}\right)} \\
& L^{\prime \prime}=\iota^{\prime \prime}\left(W\left(n^{\prime}\right) \otimes W(1)^{\left(n^{\prime \prime}\right)}\right) \otimes(\underset{\kappa}{M+N})^{\left(n^{\prime}\right)} \otimes Z^{\left(n^{\prime \prime}-1\right)} \otimes N
\end{aligned}
$$

where $\iota^{\prime}$ and $\iota^{\prime \prime}$ denote a homomorphisms of corresponding modules into $W(n)$ induced by natural injections of groups which may be assumed to be injective. 
We take an element $w$ which is an $S(n)$-base of $L^{\prime}$ written in the form

$$
w=\iota^{\prime}\left(\eta^{\prime} \otimes \eta^{\prime \prime}\right) \otimes w^{\prime} \otimes\left(\xi_{1} \otimes w_{1}\right) \otimes \cdots \otimes\left(\xi_{n^{\prime \prime}} \otimes w_{n^{\prime \prime}}\right)
$$

where $\eta^{\prime}, \eta^{\prime \prime}, w^{\prime}$ and $\xi_{\nu} \otimes w_{\nu}$ for $1 \leqq \nu \leqq n^{\prime \prime}$ are basis of the modules $W\left(n^{\prime}\right), W\left(n^{\prime \prime}\right)$, $\left(\begin{array}{c}M+N \\ \kappa\end{array}\right)^{\left(n^{\prime}\right)}$ and $\bar{N}(m)$ respectively. Then we define a homomorphism $\chi: L^{\prime} \rightarrow L^{\prime \prime}$ by the formula. Here $c$ denotes a natural homomorpism

$$
\begin{gathered}
\chi(w)=\varepsilon \iota^{\prime \prime}\left(\eta^{\prime} \otimes 1\right) \otimes w^{\prime} \otimes 1^{\left(n^{\prime \prime}-1\right)} \otimes \rho\left(\iota\left(\eta^{\prime \prime} \otimes \xi_{1} \otimes \cdots \otimes \xi_{n^{\prime \prime}}\right) \otimes w_{1} \otimes \cdots \otimes w_{n^{\prime \prime}}\right) \\
\iota: W\left(n^{\prime \prime}\right) \otimes W(m)^{\left(n^{\prime \prime}\right)} \rightarrow W\left(m n^{\prime \prime}\right)
\end{gathered}
$$

induced by the injection $S\left(m ; n^{\prime \prime}\right) \rightarrow S\left(m n^{\prime \prime}\right)$ which may be assumed to be injective too, where $S\left(m ; n^{\prime \prime}\right)$ denotes a split extension of $S(m)^{n^{\prime \prime}}$ by $S\left(n^{\prime \prime}\right)$ as introduced in Steenrod [18]. Furthermore, $\varepsilon$ denotes the signature resulting from interchanging the factors, that is

$$
\varepsilon=(-1)^{\left|\eta^{\prime \prime}\right|\left|w^{\prime}\right|+}{ }_{1 \leqq \lambda<\mu \leqq n^{\prime \prime}}\left|\xi_{\mu}\right|\left|w_{\lambda}\right| .
$$

Suppose given a set of homomorphisms $g_{\nu}: N \rightarrow K^{*}$ for $1 \leqq \nu \leqq n$ such that $g_{\nu} \kappa \sim 0$. Then there exists a trivial extension $g_{\nu}^{\prime}: M+N \rightarrow K^{*}$ of $g_{\nu}$ satisfying the relation $\delta g_{\nu}^{\prime} v=g_{\nu}$. Clearly an indexed set $\left\{g_{\nu}^{\prime}\right\}_{1 \leqq \nu \leqq n}$ of homomorphisms $g_{\nu}^{\prime}$ induces. a homomorphism

$$
g^{\prime \prime}: W(n) \otimes(\underset{\kappa}{M+N})^{(n)} \rightarrow W(n) \otimes K^{*(n)} .
$$

Let $\varphi: W(n) \otimes K^{*(n)} \rightarrow K^{*}$ be a Steenrod operation. Then we have a homomorphism

$$
\tilde{g}: W(n) \otimes(\underset{\kappa}{M+N})^{(n)} \rightarrow K^{*}
$$

as a composition of $g^{\prime \prime}$ and $\varphi$.

Now we set $N=\bar{N}(m n)$. Suppose given a set of homomorphisms $f_{\nu}: L(p) \rightarrow K^{*}$ for $1 \leqq \nu \leqq n$. Then we use the same notation $f_{\nu}: N \rightarrow K^{*}$ for the Steenrod operation induced by $f_{\nu}$. Above argument shows that those homomorphisms induce a homomorphism

$$
\tilde{f:} \tilde{L} \rightarrow K^{*} \text {. }
$$

From now on, we shall consider the case all $f_{\nu}$ 's are equal to each other. Then we can show the existence of a homotopy $\tilde{h}: L^{\prime} \rightarrow K^{*}$ such that

$$
\delta \tilde{h}+\tilde{h} \delta=\tilde{f} \mid L^{\prime}-\left(\tilde{f} \mid L^{\prime \prime}\right) x
$$

which can be taken to be functorial.

We denote by $R$ an $S(n)$-submodule of $\tilde{L}$ generated by the elements $x(w-\chi(w))$ where $x \in S(n)$ and $\left(n^{\prime}, n^{\prime \prime}\right)$ runs over the set of all the pairs such that $n^{\prime}+n^{\prime \prime}=n$. Let $R^{\prime}$ be a submodule of $\widetilde{L}$ which is not closed with respect to the coboundary operator and generated by the elements of the form

$$
c^{\prime \prime}\left(\eta^{\prime} \otimes 1\right) \otimes w^{\prime} \otimes 1^{\left(n^{\prime \prime}-1\right)} \otimes w_{1}^{\prime}
$$

where the symbols $\eta^{\prime}, w^{\prime}$ etc. mean the elements introduced in the definition of $L^{\prime \prime}$ with a supplementary property that $\eta^{\prime} \otimes w^{\prime}$ is not contained in any submodule 
of $W\left(n^{\prime}\right) \otimes(M+N)^{\left(n^{\prime}\right)}$ which is the image of $W\left(n_{1}^{\prime}\right) \otimes W\left(n_{2}^{\prime}\right) \otimes(M+N)^{\left(n_{1}^{\prime}\right)} \otimes \bar{N}(m)^{\left(n_{2}^{\prime}\right)}$ with $n_{1}^{\prime}+n_{2}^{\prime}=n^{\prime}$ by the homomorphism $\chi$. From the definition follows a direct sum decomposition

$$
\widetilde{L}=R+R^{\prime} \text {. }
$$

Finally we define the universal module $L(\kappa ; m, n)$ to be the quotient $\tilde{L} \otimes_{S(n)} Z \mid R \otimes_{S(n)} Z$ which will be denoted by $L$.

Now we define an $S(n)$-homotopy $h: R \rightarrow K^{*}$ by the formule

$$
h(\tilde{w})= \begin{cases}\tilde{h}(w) & \text { if } \tilde{w}=w-\chi(w) \text { with } w \text { defined as above, } \\ 0 & \text { if } \tilde{w} \text { is an element of } R^{\prime} .\end{cases}
$$

Setting $\tilde{f^{\prime}}=\tilde{f}-(\delta h+h \delta)$, we have a homomorphism

$$
\hat{f}^{\prime}: \widetilde{L} \rightarrow K^{*}
$$

satisfying the condition $\tilde{f^{\prime}} \mid R=0$. Obviously $\tilde{f^{\prime}}$ gives ries to a homomorphism

$$
f: L \rightarrow K^{*} \text {. }
$$

Thus obtained homomorphism $f$ plays a role of Steenrod operation for the secondary cohomology operation. It will be called the stable Steenrod operation in the sequel.

\section{$\S 4$. The constrtction of $L(p, \kappa, q ; m, n)$}

In the preceding section, we have defined a stable universal module $L(\kappa ; m, n)$ and the stable Steenrod operation $\varphi: L(\kappa ; m, n) \rightarrow K^{*}$ with some properties of secondary cohomology operations. Nevertheless these operations can not be identified with cohomology operations in the usual sense. To obtain a combinatorial definition of cohomology operations in the usual sense, we have to make another step.

From now on, $K$ will denote a Kan complex. Then the theorem of Hopf shows that an arbitrary operation

$$
\varphi: W(n) \otimes L(p)^{(n)} \rightarrow K^{*}
$$

can be factored into two homomorphisms

$$
\varphi_{0}: W(n) \otimes L(p)^{(n)} \rightarrow K(N, p)^{*}
$$

and

$$
\varphi_{1}: K(N, p)^{*} \rightarrow K^{*} .
$$

On the other hand, in Chapter 2, we have shown that there exists a submodule $W^{+}(m, p)$ of $W(m)=W^{+}(m, \pm)$ accordingly $(-1)^{p}= \pm 1$ for which we have an isomorphism

$$
\phi:{ }_{m} A^{\prime}(N, p)^{*} \rightarrow W^{+}(m, p) \underset{S(m)}{\otimes} M(p)^{(m)} .
$$

Furthermore, we can varify that the composition of $\phi$ with the operation

$$
\varphi: W^{+}(m, p) \underset{S(m)}{\otimes} M(p)^{(m)} \rightarrow_{m} A^{\prime}(N, p)^{*}
$$

gives a cochain homotopy equivalence of ${ }_{m} A^{\prime}(N, p)^{*}$. As a direct consequence 
of these facts $\phi \varphi$ gives also an equivalence of $W^{+}(m, p) \underset{S(m)}{\otimes} M(p)^{(m)}$. Therefore, if we confine curselves to the category of Kan complexes, it suffices to consider the restriction of $\varphi$ on the submodule $W^{+}(m, p) \underset{S(m)}{\otimes} M(p)^{(m)}$ instead of $\varphi$ itself.

These circumstances lead us to the following definitions of the unstable universal modules and operations.

Let $\bar{N}(m, p)$ be a module defined by

$$
\bar{N}(m, p)=\sum_{l^{\prime}+l^{\prime \prime}=m}\left(W^{+}\left(l^{\prime}, p\right) \otimes W(1)^{\left(l^{\prime \prime}\right)}\right) \sum_{S\left(l^{\prime}\right) \times S\left(l^{\prime \prime}\right)}\left(M(p)^{\left(l^{\prime}\right)} \otimes M(0)^{\left(l^{\prime \prime}\right)} .\right.
$$

We abbreviate $\bar{N}(\infty, \underset{\sim}{p})$ simply as $N$. Furthermore $M$ will denote $M(q)$. Then we define a module $\widetilde{L}(p, \kappa, q ; n)$ or ${ }_{n} \widetilde{L}$ by

$$
\widetilde{L}(p, \kappa, q ; n)=W^{+}(n, \pm) \otimes(\underset{\kappa}{M+N})^{(n)}
$$

which will be denoted by $\tilde{L}$, where \pm means the signeture of $(-1)^{q}$. In the following, $W(n)$ will denote $W^{+}(n, \pm)$ and so on.

In the sequl, we shall denote by $\varphi: N \rightarrow A(N, p)^{*}$ a Steenrod operation and by $\psi: A(N, p)^{*} \rightarrow N$ a nomotopy inverse of $\varphi$ as defined in $\S 5$, Chapter 2 .

We consider the two submodules $L^{\prime}, L^{\prime \prime}$ of $\widetilde{L}$ defined as follows :

$$
\begin{aligned}
& L^{\prime}=\iota^{\prime}\left(\left(W\left(n^{\prime}\right) \otimes W\left(n^{\prime \prime}\right)\right) \otimes(\underset{\kappa}{M+N})^{\left(n^{\prime}\right)} \otimes \bar{N}(m, p)^{\left(n^{\prime \prime}\right)}\right. \\
& L^{\prime \prime}=\iota^{\prime \prime}\left(W\left(n^{\prime \prime}\right) \otimes W(1)^{(n)}\right) \otimes(\underset{\kappa}{M+N})^{\left(n^{\prime}\right)} \otimes Z^{\left(n^{\prime \prime}-1\right)} \otimes N
\end{aligned}
$$

where the notations are the same as in $\S 3$.

Let $w$ be an $S(n)$ free base of $L^{\prime}$ written in the form

$$
w=\iota^{\prime}\left(\eta^{\prime} \otimes \eta^{\prime \prime}\right) \otimes w^{\prime} \otimes \xi_{1} \otimes w_{1} \otimes \otimes \cdots \otimes \xi_{n^{\prime \prime}} \otimes w_{n^{\prime \prime}}
$$

Now the homomorphism $\chi: L^{\prime} \rightarrow L^{\prime \prime}$ is defined by

$$
\chi(w)=\varepsilon \iota^{\prime \prime}\left(\eta^{\prime} \otimes 1\right) \otimes w^{\prime} \otimes 1^{\left(n^{\prime \prime}-1\right)} \otimes \pi\left(\iota\left(\eta^{\prime} \otimes \xi_{1} \otimes \cdots \otimes \xi n^{\prime \prime}\right) \otimes w \otimes \cdots \otimes w n^{\prime \prime}\right)
$$

where $\iota: W\left(n^{\prime}\right) \otimes W(m)^{\left(n^{\prime}\right)} \rightarrow W\left(n^{\prime} m\right)$ denotes an injection defined in $\S 3$. and $\pi: W(n m) \rightarrow W^{+}(n m, p)$ denotes a retraction homotopic to $\phi \varphi$.

Let $R$ be an $S(n)$-submodule of $\widetilde{L}$ generated by the elements $x(w-\chi(w))$ where $x \in S(n)$ and $\left(n^{\prime}, n^{\prime \prime}\right)$ runs over the set of all the pairs of nonnegative integers such that $n^{\prime}+n^{\prime \prime}=n$.

Now we define the universal module $L$ to be the quotient $\tilde{L} \otimes_{S(n)} Z / R \otimes_{S(n)} Z$.

Suppose given a homomorphism $f: L(p) \rightarrow K^{*}$ such that the induced Steenrod operation $f: N \rightarrow K^{*}$ satisfies the relation $f \kappa \sim 0$. Then $f$ can be extended to a homomorphism $\hat{f}: \widetilde{L} \rightarrow K^{*}$

In the sequel, we assume the commutatity of the following diagram for a Steenrod operation

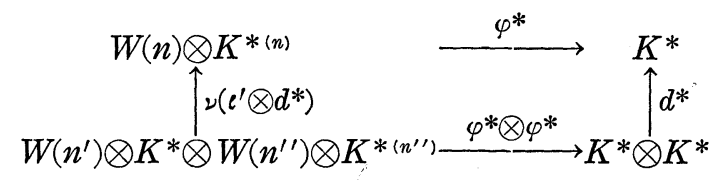

where $\nu$ denotes a permutation of factors. Furthermore we shall restrict ourselves 
to the case $K=K(p, k, q)$ where $g \kappa \sim k$ for homomorphism $g: N \rightarrow K(Z, p)^{*}$ such that $g u=u$.

Under these assumptions, we shall construct a homomorphism ${ }_{n} \tilde{f}^{\prime}:{ }_{n} \widetilde{L} \rightarrow K^{*}$ and a homotopy ${ }_{n} h ;{ }_{n} \widetilde{L} \rightarrow K^{*}$ satisfying the following conditions :

i) $\delta h+h \delta={ }_{n} \tilde{f}-{ }_{n} \tilde{f}^{\prime}$

ii) Let $\tilde{\iota}:{ }_{n^{\prime}} \widetilde{L} \otimes W\left(n^{\prime \prime}\right) \otimes \bar{N}(m, p)^{\left(n^{\prime \prime}\right)} \rightarrow{ }_{n} \widetilde{L}$ be the natural injection whose image is a ${ }_{n} \widetilde{L}^{\prime}$. Then we have

$$
{ }_{n} \tilde{f^{\prime}} \tilde{\iota}\left(\tilde{w}^{\prime} \otimes \tilde{w}^{\prime \prime}\right)={ }_{n^{\prime}} \tilde{f}^{\prime}\left(\tilde{w}^{\prime}\right) \cup \tilde{f^{\prime}}\left(\tilde{w}^{\prime \prime}\right)
$$

for $\tilde{w}^{\prime} \in{ }_{n^{\prime}} \widetilde{L}$ and $\tilde{w}^{\prime \prime} \in W\left(n^{\prime \prime}\right) \otimes \bar{N}(m, p)^{\left(n^{\prime \prime}\right)}$.

In case $n^{\prime}=0$, we have

$$
{ }_{n} \tilde{f}^{\prime}\left(\eta \otimes \xi_{1} \otimes w_{1} \otimes \cdots \otimes \xi_{n} \otimes w_{n}\right)=\varepsilon \tilde{f}^{\prime}\left(\pi \iota\left(\eta \otimes \xi_{1} \otimes \cdots \otimes \xi_{n}\right) \otimes w_{1} \otimes \cdots \otimes w_{n}\right)
$$

where $\eta \in W(n)$ and $\xi_{r} \otimes w_{r} \in \bar{N}(m, p)$ for $1 \leqq r \leqq n$.

iii) The homotopy ${ }_{n} h$ is defined so as to

$$
{ }_{n} h \tilde{\iota}\left(\tilde{w}^{\prime} \otimes \tilde{w}^{\prime \prime}\right)=\omega \cdot{ }_{n}, \hat{f}\left(\tilde{w}^{\prime}\right) \cup_{n^{\prime \prime}} h\left(\tilde{w}^{\prime \prime}\right)+{ }_{n}, h\left(\tilde{w}^{\prime}\right) \cup_{n^{\prime}}, \tilde{f}^{\prime}\left(\tilde{w}^{\prime \prime}\right)
$$

where $\omega$ denotes an involution defined by $\omega(u)=(-1)^{\operatorname{dim} u} u$. The injectivity of $\iota^{\prime}$ and the associativity of the cup product assures the existence of the homomorphisms ${ }_{n} \tilde{f}^{\prime}$ satisfying the required propertics.

Clearly the homomorphism $\tilde{f}^{\prime}: \widetilde{L} \rightarrow K^{*}$ satisfies the condition $\tilde{f}^{\prime} \mid R=0$, hence this induces a homomorphism

$$
f: L \rightarrow K^{*}
$$

which will be called a Steenrod operation.

\section{$\S 5$. The construction of the homomorphism $\psi(p, \kappa, q ; m, n)$}

Suppose given a characteristic homomorphism

$$
T: A(N, p) \rightarrow A(N, q+1) \text {. }
$$

Then we can define a twisted tensor product $A(p, T, q)=A(N, q) \otimes A(N, p)$. Here we recall the coboundary formula of $A(p, T, q)^{*}=A(N, q)^{*} \underset{T^{*}}{\otimes} A(N, p)^{*}$. Let $\alpha \otimes \beta \in A(p, T, q)^{*}$ with $\alpha \in A(N, q)^{*}$ and $\beta \in A(N, p)^{*}$. Then we have

$$
\begin{aligned}
& \delta(\alpha \otimes 1)=\delta \alpha \otimes 1+\sum(-1)^{\left|\alpha^{\prime}\right|} \alpha^{\prime} \otimes T^{*}\left[\alpha^{\prime \prime}\right] \\
& \delta(\alpha \otimes \beta)=\delta(\alpha \otimes 1) \cdot \beta+(-1)^{|\alpha|} \alpha \otimes \delta \beta
\end{aligned}
$$

where the summation in the first formula is extended over the set of all the pairs $\left(\alpha^{\prime}, \alpha^{\prime \prime}\right)$ such that $m^{*} \alpha=\sum \alpha^{\prime} \otimes \alpha^{\prime \prime}$ while $m$ is a multiplication of $A(N, q)$.

Let $\varphi: \bar{N}(\infty, p) \rightarrow A(N, p)^{*}$ be a Steenrod operation and let $\phi: A^{*}(N, p)^{*} \rightarrow \bar{N}(\infty, p)$ be the homotopy inverse of $\varphi$ as defined in $\S 5$, Chap 2. Let $L^{\prime}$ denote $L(q+1)$ and $v^{\prime}$ denate the generator of $L^{\prime}$ in dimension $q+1$. Clearly we have $M+L_{v^{\prime}} \cong$ $L(0, q)_{N}^{*}$. Let $\tilde{\psi}: B(N, q, n)^{*} \rightarrow W(n) \underset{S(n)}{\otimes}\left(M_{\kappa}+L^{\prime}\right)^{(n)}$ be the homomorphism defined in also $\S 5$, Chap 2 .

Now we define a homomorphism

$$
\tau: W(n) \underset{S(n)}{\otimes}\left(M+{ }_{v^{\prime}} L^{\prime}\right)^{(n)} \rightarrow L(p, k, q ; m, n)
$$


to be natuarl projection given by $\tau \tilde{v}=v$ where $\tilde{v}$ denotes a generator of $M$ in dimension $q$.

Let us put $k=T^{*} \tilde{\delta} v$ with the fundamental cocycle $\tilde{\delta} v$ of $A(N, q+1)^{*}$ and $\kappa=\psi k$. Here we assume $\kappa \in \bar{N}(m)^{p}$. Then Corollary 2.5 implies that the characteristic homomorphism $T$ can be assemed to be defined so as to satisfy the formula

$$
T^{*}=\varphi \tau \tilde{\psi}
$$

on the direct summand $A(N, q+1, n)^{*}$ of $A(N, q+1)^{*}$.

These assumptions allow us to obtain a submodule $A(p, T, q ; n)^{*}$ of $A(p, T, q)^{*}$ consisting of elements $\alpha \otimes \beta$ such that $\mu \alpha \leqq n$.

We shall show that $\phi$ can be extended to a homomorphism

$$
\phi: A(p, k, q ; n)^{*} \rightarrow L(p, \kappa, q ; m, n)
$$

so that the following diagram becomes homotopy commutative

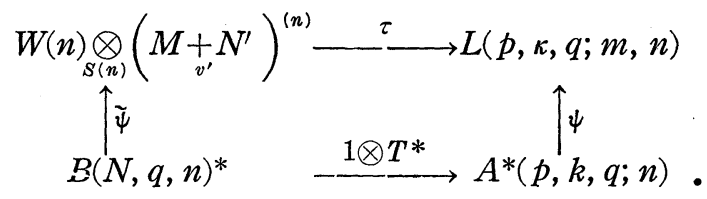

To simplify the notation, we shall use the symbol $L$ and $A^{*}$ to indicate $L(p, \kappa, q ; m, n)$ and $A(p, k, q ; n)^{*}$ respectively. Let $A^{*}(s)$ be a submodule of $A^{*}$ generated by the elements of the form $\alpha \otimes \beta$ with the property

$$
\frac{1}{2} \mu(\alpha)(\mu(\alpha)-1) q+\mu(\alpha) \cdot(q-\operatorname{dim} \alpha) \leqq s .
$$

From the coboundary formula, we can see that $A^{*}(s)$ defines a filtration on $A^{*}$. In the same way, we define $B^{*}(s)$ to be the submodule of $B(N, q)^{*}$ generated by the elements of the form $\alpha \otimes \beta$ with the same property. The construnction of $\phi$ is given now by induction on this filtration. To begin with, the definition of the universal module enables us to obtain a homotopy $h: A(N, q+1, n)^{*} \rightarrow N$ satisfying $\delta h+h \delta=\phi T^{*}-\tau \tilde{\psi}$. Hence we assume that $\phi$ is already defined on $A^{*}\left(\frac{1}{2} n(n-1) q+r-1\right)$ with $0 \leqq r \leqq n q$ such that there exists a homotopy $h: B^{*}\left(\frac{1}{2} n(n-1) q+r-1\right) \rightarrow L$ satisfying $\delta h+h \delta=\phi\left(1 \otimes T^{*}\right)-\tau \tilde{\psi}$.

Let $\alpha \otimes \beta \in A^{*}\left(\frac{1}{2} n(n-1) q+r\right)$ not belonging to $A^{*}\left(\frac{1}{2} n(n-1) q+r-1\right)$. If $\beta=1$, then $\phi(\alpha \otimes 1)$ is defined as fullows. We use the symbol $\tilde{\delta}$ to indicate the coboundary operator in $B(N, q, n)^{*}$. Then the definition of the bar-construction implies $\tilde{\delta}(\alpha \otimes 1)=\delta \alpha \otimes 1+\sum(-1)^{\left|\alpha^{\prime}\right|} \alpha^{\prime} \otimes\left[\alpha^{\prime \prime}\right]$ where the summation is extended over the same pairs $\left(\alpha^{\prime}, \alpha^{\prime \prime}\right)$ as before. Since $\operatorname{dim} \alpha^{\prime \prime}>0$ except when $\alpha^{\prime \prime}=1$, we have $\operatorname{dim}\left[\alpha^{\prime \prime}\right] \geqq 2$. Hence it follows that $\mu \alpha^{\prime \prime}>0$ and $\mu \alpha^{\prime}<\mu \alpha=n$.

Then we have $\tilde{\delta}(\alpha \otimes 1) \equiv \delta \alpha \otimes 1 \bmod B^{*}\left(\frac{1}{2} n(n-1) q\right)$ and consequently $\widetilde{\delta}(\alpha \otimes 1) \equiv 0$ $\bmod B^{*}\left(\frac{1}{2} n(n-1) q+r-1\right)$. Therefore the inductive hypothesis provides an element $h \tilde{\delta}(\alpha \otimes 1)$ satisfying the relation $\delta h \tilde{\delta}(\alpha \otimes 1)=\left(\psi\left(1 \otimes T^{*}\right)-\tau \tilde{\psi}\right) \tilde{\delta}(\alpha \otimes 1)$. Now we put $\phi(\alpha \otimes 1)=(\tau \tilde{\phi}+h \tilde{\delta})(\alpha \otimes 1)$ and $h(\alpha \otimes 1)=\varepsilon(\alpha)$. Since $\delta \tau \tilde{\phi}=\tau \tilde{\phi} \tilde{\delta}$, we have $\delta \phi(\alpha \otimes 1)=$ $\phi\left(1 \otimes T^{*}\right) \tilde{\delta}(\alpha \otimes 1)=\psi \delta(\alpha \otimes 1)$. If $\beta \neq 0$ is an element such that $\phi(\beta)=w^{\prime \prime} \in N$, then 
$\phi(\alpha \otimes 1)=\eta^{\prime} \otimes w^{\prime}$ is assumed to be contained in $L(p, \kappa, q ; m, n-1)$. Now $\phi(\alpha \otimes \beta)$ is defined by $\iota^{\prime}\left(\eta^{\prime} \otimes 1\right) \otimes w^{\prime} \otimes w^{\prime \prime}$. Again from our construction, we have $\delta \phi(\alpha \otimes \beta)=$ $\phi \delta(\alpha \otimes \beta)$. Finally the formula $h(\alpha \otimes \beta)=\varepsilon(\alpha) \cdot h(1 \otimes \beta)$ for $\alpha \otimes \beta \in B^{*}\left(\frac{1}{2} n(n-1) q+r\right)$ gives us a homotopy with the required property.

Let $F_{l}$ be the submodule of $A^{*}$ generated by the elements of the form $\alpha \otimes \beta$ with $\mu(\alpha) \leqq l$. Then $F_{l}$ defines a filtration on $A^{*}$. In the same way, we define $\widetilde{F}_{l}$ to be the submodule of $B(N, q)^{*}$ generated by the elements of the form $\alpha \otimes \beta$ with $\mu(\alpha) \leqq l$.

Let $G_{l}$ be the submodule of $L$ generated by the element of the form $\eta \otimes v^{\left(l^{\prime}\right)} \otimes w$ with $l^{\prime} \leqq l$ and $w \in N^{\left(n-l^{\prime}\right)}$. Then $G_{l}$ defines a filtration on $L$. In the same way, we define $\tilde{G}_{l}$ to be the submodule of $W(n) \underset{S(n)}{\otimes}\left(M+L_{v^{\prime}} L^{\prime}\right)^{(n)}$ generated by the elements of the form $\eta \otimes v^{\left(l^{\prime}\right)} \otimes w$ with $l^{\prime} \leqq l$ and $w \in L^{\prime\left(n-l^{\prime}\right)}$.

Lemma $3.5 \phi F_{l} \subset G_{l}$.

Proof. Take an element $\alpha \otimes \beta \in F_{l}$. If $\beta=1$, it is sufficient to show $\phi(\alpha \otimes 1) \equiv$ $\phi \alpha \otimes 1 \bmod G_{l-1}$. In fact, by the construction of $\tilde{\psi}$ using the cell decomposition of $\mathrm{SP}^{n} D^{q+1}$ given in $\S 5$, Chap 2 , we can assume $\widetilde{\phi}(\alpha \otimes 1) \equiv \phi \alpha \otimes 1 \bmod \widetilde{G}_{l-1}$. Thus $\tau \widetilde{G}_{l-1} \subset G_{l-1}$ yields $\tau \widetilde{\psi} \equiv \phi \alpha \otimes 1 \bmod G_{l-1}$. On the other hand, the properties of $h$ imply $h \widetilde{\delta}(\alpha \otimes 1)=h[\alpha] \in G_{l-1}$. Applying these facts to the formula $\phi(\alpha \otimes 1)=$ $(\tau \tilde{\psi}+h \tilde{\delta})(\alpha \otimes 1)$, we obtain $\phi \alpha(\otimes 1) \equiv \phi \alpha \otimes 1 \bmod G_{l-1}$. If $\alpha \otimes \beta \in F_{l}$ i.e. $\mu(\alpha) \leqq l$, the formula for $\psi(\alpha \otimes \beta)$ leads to the required result $\psi(\alpha \otimes \beta) \equiv \phi \alpha \otimes \psi \beta \bmod G_{l-1}$.

From the proof of Lemma 3.5 follows

Lemma 3.6 For $\alpha \otimes \beta \in F_{l}$, we have

$$
\psi(\alpha \otimes \beta) \equiv \phi \alpha \otimes \psi \beta \bmod G_{l-1}
$$

Applying the argument of acyclic models we can define a Steenrod operation for which we have the following two lemmas:

LEMMA 3.7

$$
\varphi G_{l} \subset F_{l} .
$$

Lemma 3.8 For $w=\iota^{\prime}\left(\eta^{\prime} \otimes \eta^{\prime \prime}\right) \otimes v^{\left(l^{\prime}\right)} \otimes w^{\prime \prime} \in G_{l}$ with $\eta^{\prime \prime} \in W\left(n-l^{\prime}\right)$ and $w^{\prime \prime} \in N^{\left(n-l^{\prime}\right)}$, we have

$$
\varphi(w) \equiv \pm \varphi\left(\eta^{\prime} \otimes v^{\left(l^{\prime}\right)}\right) \cdot \varphi\left(\eta^{\prime \prime} \otimes w^{\prime \prime}\right) \bmod F_{l-1}
$$

Thus we finally arrive at our Main Theorem which asserts the equivalence of the two definitions of cohomology operations.

TheOREM 3.9 Let

$$
\varphi: L(p, \kappa, q ; m, n) \rightarrow A(p, k, q: n)^{*}
$$

be a homomorphism defined satifying Lemmas 3.7 and 3.8, and

$$
\phi: A(p, k, q ; n)^{*} \quad \rightarrow L(p, \kappa, q ; m, n)
$$

be a homomorphism defined in $\S 5$. Then the composition of these two homomorphisms

$$
\varphi \psi: A(p, k, q ; n)^{*} \rightarrow A(p, k, q ; n)^{*}
$$

gives a homotopy equivalence. 
To prove the Theorem, it suffices to see that $\varphi \psi$ gives an isomorphism of $E_{2}$ term. This fact can be assured by Lemmas 3.6, 3.8.

\section{References}

[1] Adem. J., The relations on Steenrod power of cohomology classes, Algebraic geometry and topology (A symposium in honor of Lefschetz), (Princeton University Press, 1956).

[2] Brown, E. H., Finite computability of Postnikov complexes, Annals of Math., 65, 1-20, (1957).

[ 3 ] — Twisted tensor products; I, Annals of Math., 69, 223-246. (1959).

[4] Cartan, H., Séminaire H. Cartan, 1954-1955, Paris.

[5] Dold, A., Homology of symmetric products and other functors of complexes, Annals of Math., 68, 54-80, (1958).

[6 ] - Ueber die Steenrodschen Kohomologieoperationen, Annals of Math., 73, 258-294, (1961).

[ 7 ] - Decomposition theorems for $S(n)$-complexes, Annals. of Math., 75, 8-16, (1962).

[8] Dold, A. and Thom, R., Quasifaserungen und unendliche symmetrische Produkte, Annals of Math., 67, 239-281, (1958).

[9] Eilenberg, S. and MacLane, S., On the groups $H(\Pi, n)$, I-III, Annals of Math., 58, 55-106, (1953); 60, 49-139, (1954); 60, 513-557, (1954).

[10] Moore, J., Seminar notes, 1955/1957, Princeton University.

[11] Nakamura, T., On the homology groups of Eilenberg-MacLane complexes, Sûgaku, 7, 89-98, (1955) (in Japanese).

[12] - Equivalence between two definitions of the cohomology operations, Scientific Papers of the College of General Education Tokyo Univ., 1-16, 9, (1959).

[13] Nakaoka, M., Cohomology of symmetric pooducts, J. Inst. Polyt. Osaka City Univ., 8, 121-144, (1957).

[14] - Homology of the infinite symmetric group, Annals of Math., 73, 229-257, (1961).

[15] Peterson, F. P and Stein, N., Secondary cohomology operations; two formulas, Amer. J. Math., 81, 281-305, (1951).

[16] Serre, J. P., Cohomologie modulo 2 des complexes d'Eilenberg-MacLane, Comment. Math. Helv., 27, 193-231, (1953).

[17] Shimada, N. and Yamanoshita, T., On triviality of the mod $p$ Hopf invariant, Jap. J. of Math., 31, 1-26, (1961).

[18] Steenrod, N. E., Cohomology operations derived from the symmetric group, Comment. Math. Helv., 32, 129-152, (1958).

[19] _- Lecture notes (1957), Princeton University.

[20] Steenrod, N. E. and Thomas, P. E. Cohomology operations derived from the cyclic group., Comment. Math. Helv., 32, 129-152, (1958).

[21] Szczarba, R. H. The homology of twisted cartesian products, Trans. of Amer. Math. Soc., 100, 197-216, (1961).

[22] Thom, R., Strasbourg Colloq., (mimeographed), (1951). 